\title{
BCS instability and finite temperature corrections to tachyon mass in intersecting $D 1$-branes
}

\author{
Sudipto Paul Chowdhury, ${ }^{a}$ Swarnendu Sarkar ${ }^{b}$ and B. Sathiapalan ${ }^{a}$ \\ ${ }^{a}$ The Institute of Mathematical Sciences, \\ Taramani, Chennai 600113, India \\ ${ }^{b}$ Department of Physics and Astrophysics, University of Delhi, \\ Delhi 11000\%, India \\ E-mail: sudiptopc@imsc.res.in, ssarkar@physics.du.ac.in, \\ bala@imsc.res.in
}

Abstract: A holographic description of BCS superconductivity is given in [1]. This model was constructed by insertion of a pair of $D 8$-branes on a $D 4$-background. The spectrum of intersecting $D 8$-branes has tachyonic modes indicating an instability which is identified with the BCS instability in superconductors. Our aim is to study the stability of the intersecting branes under finite temperature effects. Many of the technical aspects of this problem are captured by a simpler problem of two intersecting D1-branes on flat background. In the simplified set-up we compute the one-loop finite temperature corrections to the treelevel tachyon mass-squared-squared using the frame-work of SU(2) Yang-Mills theory in $(1+1)$-dimensions. We show that the one-loop two-point functions are ultraviolet finite due to cancellation of ultraviolet divergence between the amplitudes containing bosons and fermions in the loop. The amplitudes are found to be infrared divergent due to the presence of massless fields in the loops. We compute the finite temperature mass-squared correction to all the massless fields and use these temperature dependent masses-squared to compute the tachyonic mass-squared correction. We show numerically the existence of a transition temperature at which the effective mass-squared of the tree-level tachyons becomes zero, thereby stabilizing the brane configuration.

KEYwords: Brane Dynamics in Gauge Theories, Intersecting branes models, AdS-CFT Correspondence

ARXIV EPRINT: 1403.0389 


\section{Contents}

1 Introduction $\quad 2$

2 Tree-level spectrum 5

3 Finite temperature analysis with one scalar: warm up exercise $\quad 9$

4 Intersecting D1 branes at finite temperature $\quad 14$

$\begin{array}{lll}4.1 \text { Bosons } & 15\end{array}$

$\begin{array}{llr}4.2 & \text { Fermions } & 19\end{array}$

5 The ultraviolet and infrared problems $\quad \mathbf{2 3}$

5.1 Ultraviolet finiteness of tachyonic amplitudes 24

$\begin{array}{ll}5.2 & \text { Infrared problem } \\ 5.3 & \end{array}$

5.3 Two-point functions for the $\tilde{C}_{w, k}$ modes 31

$\begin{array}{ll}5.4 & \text { Two point function for } \Phi_{1}^{3} \\ 5.5 & 35\end{array}$

5.5 Two point function for $\Phi_{I}^{3}(I \neq 1) \quad 40$

5.6 Two point function for $A_{x}^{3} \quad 44$

6 Finite part of effective tachyon mass 48

6.1 Numerical results 48

$\begin{array}{lll}7 & \text { Discussion and outlook } & 53\end{array}$

A Dimensional reduction of $D=10, \mathcal{N}=1, \mathrm{SU}(2) \mathrm{SYM}$ to $D=2 \quad 54$

B Tables of fields, eigenfunctions and normalizations $\quad 56$

C Propagators and vertices for computation of two-point $C_{w, k}$ amplitudes $\mathbf{5 8}$

$\begin{array}{lll}\text { C.1 Bosons } & 58\end{array}$

$\begin{array}{lll}\text { C.2 Fermions } & 61\end{array}$

D Vertices for computation of two-point $\tilde{C}_{w, k}$ amplitudes $\quad 63$

$\begin{array}{lll}\text { D.1 Bosonic vertices } & 63\end{array}$

$\begin{array}{lll}\text { D.2 } & \text { Fermionic vertices } & 64\end{array}$

E Vertices for computation of two point amplitudes for $\Phi_{I}^{3}$ and $A_{x}^{3} \quad 67$

$\begin{array}{lll}\text { E.1 } \Phi_{1}^{3} \text { vertices } & 67\end{array}$

E.2 $\Phi_{I}^{3}, I \neq 1$ vertices $\quad 69$

$\begin{array}{lll}\text { E.3 } & A_{x}^{3} \text { vertices } & 70\end{array}$

$\begin{array}{lll}\text { F } & \text { Matsubara sums } & 73\end{array}$ 


\section{Introduction}

There have been many applications of the AdS/CFT correspondence to understand condensed matter systems. When there are gapless modes present these systems are described by conformal field theories at low energies. This can happen at second order phase transitions, but also in metals where the excitations above the Fermi surface are gapless. For recent developments see [2]-[9].

At low temperatures metals are unstable towards electron Cooper pair formation and an energy gap develops. This is the BCS instability. The Cooper pairs are charged and so the condensate breaks the $\mathrm{U}(1)$ of electromagnetism and the photon effectively becomes massive as a result of the Higgs phenomenon. The energy gap ensures that at low frequencies there is no dissipation of energy when a current flows. The mass of the photon results in the exponential fall off of the magnetic field inside a superconductor. These are typical characteristics of superconductors. Studies of various types of superconductors using holographic techniques have been been the subject of research for the past few years. A partial list of references is $[10]-[12,13]$.

Inspired by this BCS phenomenon Nambu and Jona-Lasinio gave a description of chiral symmetry breaking in strong interactions [14, 15]. Their starting point was a non renormalizable model with four Fermi interactions. The pairing between quarks and antiquarks is analogous to Cooper pairing. The main point of difference (as summarized in [1]) is that due to the absence of a Fermi surface this instability in QCD happens only for large enough coupling. Another point of difference is that the resultant condensate breaks an axial symmetry (rather than a vector symmetry as in BCS)- the U(1) chiral symmetry that is present in $\mathrm{QCD}$ (in the absence of bare mass to quarks).

A holographic dual of $3+1$ QCD was constructed in [16] starting from M theory on $A d S_{7} \times S^{4}$. Many interesting calculations have been done with this model including calculation of the glueball mass spectrum - albeit at strong bare coupling [17]-[22-24]. An extension of this model to include flavor degrees of freedom was constructed by Sakai and Sugimoto [25]. Various aspects of this model was further explored in [26]-[31, 32]. The flavour branes are D8 branes hanging down from the boundary (where they intersect the D4 branes) and are wrapped on $S^{4}$. It was shown that when there are D8 branes and D8 anti branes, a stable configuration is described by the brane and anti-brane bending towards each other and joining to form a continuous U-shaped brane. Since the branes describe left handed quarks and anti-branes describe right handed quarks (or left handed anti quarks) this U configuration breaks chiral symmetry.

In [1] the Sakai-Sugimoto model was modified to describe BCS superconductivity. The Sakai Sugimoto model has unbroken vector like symmetries corresponding to the flavour group. Thus for two flavours there is a $\mathrm{U}(2)$. In [1] it was shown that in the presence of a finite chemical potential for the U(1) embedded in SU(2), a D8 brane and an anti D8 brane cross each other. Such a configuration is known to be tachyonic and it has been argued that the stable configuration to which this flows has a non zero charged condensate that Higgses the U(1) symmetry [33-40]. In [1] analytical solutions were given for such systems by solving the Yang-Mills equations describing intersecting branes in flat space-time. Semi 
analytic and numerical solutions were also given in the curved background of this modified Sakai-Sugimoto model.

Being a strongly coupled system the expression for the gap in terms of the coupling and other parameters is different from weak coupling BCS. The gap and thus the transition temperature are expected to be larger here. For weak coupling the relation is $\Delta \approx \epsilon_{c} e^{-\frac{1}{g \frac{d n}{d \epsilon}}}$, whereas for strong coupling one expects $\Delta \approx \epsilon_{c} g \frac{d n}{d \epsilon}$. Here $\epsilon_{c}$ is some parameter of the metal that fixes the region around the Fermi surface that participates in the pair formation.

In this paper we attempt to calculate the transition temperature for the model described in [1]. We do this in flat space-time for simplicity. The low energy theory on the brane can be described by the DBI action for the massless fields on the brane. This is valid as long as only energies $\ll \frac{1}{\alpha^{\prime}}$ are being probed. The DBI action describes the effect of "integrating out classically" (i.e. via equations of motion) the massive modes of the string. However even if the massive modes are integrated out in the quantum theory, the resultant action for the massless modes would look like supersymmetric Yang-Mills corrected by higher dimensional operators down by powers of $\alpha^{\prime}$ - very similar to the DBI action.

We can study this as a quantum theory with a cutoff $\Lambda<\frac{1}{\sqrt{\alpha}^{\prime}}$ and proceed to study the corrections due to the massless mode quantum and thermal fluctuations. Since the YangMills action is renormalizable, we know that the effect of the irrelevant higher dimension operators is to make finite renormalizations of the lower dimensional operators. This is just a re-phrasing of the decoupling theorem: if the low energy theory is renormalizable the massive modes decouple and further the ambiguities associated with the physics at and above the cutoff scale can be absorbed into a renormalization of the parameters. While this is a consistent procedure, this is not good enough for us because if we want to estimate the finite thermal corrections to the action, the finite part should be unambiguous and cannot be the finite part of an infinite term. Fortunately, because of supersymmetry, the mass-squared corrections are finite and therefore calculable in principle. More precisely if we calculate the corrections as a power series in $\Lambda$ (with $\Lambda \ll \frac{1}{\sqrt{\alpha}}$ ), one expects that terms that diverge when $\Lambda \rightarrow \infty$ (i.e. positive powers and logarithms) are absent. Thus all corrections are finite and at most of order $\frac{E}{\Lambda}<\sqrt{\alpha^{\prime}} E$ where $E$ is a typical energy scale. Supersymmetry in fact can ensure this even if the theory is not renormalizable as in $D p$-branes with $p>3$.

The physical quantity we are interested is the temperature correction to the tachyon mass-squared. The tachyon mass-squared is $O\left(\frac{\theta}{\alpha^{\prime}}\right)=-q$, where $\theta$ is the angle of intersection of the $D$-branes and $q$ is defined more precisely later. Thus we would like to keep this finite as $\frac{1}{\alpha^{\prime}} \rightarrow \infty$. This can be achieved by taking the limit $\theta \rightarrow 0, \frac{1}{\alpha^{\prime}} \rightarrow \infty$ such that $q$ is fixed. Thus in this limit we can use the supersymmetric Yang-Mills theory on the brane. We also simplify further the problem by studying $D 1$ branes. Classically the solutions we are considering depend only on one coordinate. So the solutions are the same for all $D p$ branes. Quantum mechanically the fluctuations will be different and the momentum integrals in Feynman diagrams will be different. However many of the techniques used for $D 1$ branes should go through since the mass-squared corrections are finite due to supersymmetry.

Even with these simplifications the calculations are already quite involved. The main reason is that the background configuration about which quantum corrections need to be 
calculated is space dependent. The intersecting $D$-brane configuration is described by one of the adjoint scalars having a value that is linear in $x$, in the form $\phi=q x$. Thus in the $x$-direction one cannot use plane waves as a basis. One has to work with eigenfunctions that are essentially harmonic oscillator wave functions. One should then calculate the effective potential at finite temperature and then obtain the transition temperature. This is a rather difficult calculation. In this paper as a first step we adopt the simpler procedure of calculating the corrections to the tachyon mass-squared and finding the temperature at which this turns positive. This is not the same thing because positive (mass) ${ }^{2}$ only ensures local stability. In any case with these simplifications the calculation becomes tractable. Even so, some of the calculations have to be done numerically.

There are two technical issues that become complicated because of using Hermite polynomials instead of plane waves. One is that of showing UV finiteness. As mentioned above, if the theory has divergent mass-squared corrections that need to be renormalized then one cannot calculate the transition temperature from first principles because there is always an arbitrary parameter corresponding to finite mass renormalization. Especially when calculations have to be done numerically, one needs to be sure that the series being summed is convergent. This demonstration is made difficult, once again because we cannot use a plane wave basis. In this paper we show UV finiteness at one loop. This check is also useful because it ensures that the degrees of freedom counting has been done correctly.

The second complication is that there are many massless modes. This results in infrared divergences. The correct solution to this problem is to use a renormalization group and integrate out high momentum modes first. This should typically induce mass-squared corrections to the massless modes (unless they are protected) and the final solution to the RG equations should not have any IR divergences. The full RG is difficult to implement. However what can be done is to first do a one loop integral where the internal lines have only modes that do not generate IR divergences. In this step one can calculate the masssquared correction to all the massless modes. At the next step one includes the remaining unintegrated modes in the tachyon mass-squared correction, with corrected massive propagators and now, because there are no massless modes, there are no IR divergences. ${ }^{1}$ In practice one needs to calculate mass-squared corrections only for those modes that are needed for the tachyon mass-squared correction. Thus this procedure takes care of the infrared divergences.

Once these problems are taken care of one can proceed to a calculation of the finite temperature correction to the tachyon mass-squared. Both, the temperature independent finite quantum correction to the masses-squared and the temperature dependent finite corrections are calculated. However in the final calculation of the tachyon mass-squared, which is done numerically, only the total correction is calculated and plotted. Thus we are able to calculate numerically the transition temperature at which the tachyon becomes massive.

As mentioned above this calculation needs to be generalized to higher branes. Also instead of calculating the correction to the mass-squared one should do a more complete

\footnotetext{
${ }^{1}$ At this point one is going beyond the one loop approximation and one has in effect summed an infinite number of diagrams.
} 
calculation and calculate the effective potential. Finally one should generalize to curved space time. This last may not however be very important because most of the dynamics takes place locally at the intersection point and one should be able to make a simple extrapolation to curved space locally using the equivalence principle.

This paper is organized as follows. Section 2 is dedicated to the study of mass spectrum of intersecting D1-branes at zero temperature in the Yang-Mills approximation. We choose a background given by the vev of one of the scalar field components. We compute the normalizable eigenfunctions for all the bosonic fields. Those fields which couple to the chosen background become massive at the tree-level and have a discrete mass spectrum. Those fields which do not couple to the background remain massless with a continuous spectrum. Apart from the massive modes there are also massless modes in the expansion of some of the bosonic fields which are accompanied with eigenfunctions with zero mass eigenvalue. The lowest lying modes in the bosonic mass spectrum are the tachyons. In section 3 we present the finite temperature analysis with a single scalar field using background field method. In section 4 we present the finite temperature analysis for the intersecting D1-branes. In section 4.1 we present the computations for the one-loop bosonic corrections to the tree-level tachyon mass-squared at finite temperature. The fermionic eigenfunctions and their contributions to the one-loop corrections are presented in section 4.2. In section 5 we discuss the problems of ultraviolet and infrared divergences of the amplitudes which arise due to the presence of massless fields in the loop. While the amplitudes containing bosons in the loop are both IR and UV divergent the amplitudes containing fermions in the loop are only UV divergent. In section 5.1 we present the computations for the cancellation of the UV divergences of the amplitude for the tachyonic mode. To tackle the IR problem we first compute the one-loop corrections to all the massless fields. Thus we have all massive fields at one-loop level which then allows us to compute the finite twopoint functions for the tachyonic modes. The one-loop mass-squared corrections to all the massless fields at finite temperature are computed in the sections 5.3, 5.4, 5.5 and 5.6. We also discuss their UV finiteness. In section 6.1 we present the numerical computation of the transition temperature and an analytical estimate of the behaviour of the massessquared with varying temperature. For large values of temperature, the masses-squared are found to grow linearly with temperature. We present relevant details of the computation in the appendices.

\section{Tree-level spectrum}

In this section we study the classical mass spectrum for an intersecting $D 1$ brane configuration at zero temperature in the Yang-Mills approximation. The action for two coincident $D 1$ branes with gauge group SU(2) has been worked out in appendix A. The action (A.13) is the dimensional reduction of 10 dimensional $\mathcal{N}=1$ Super Yang-Mills. The equations of motion in $1+1$ dimensions have a solution $\Phi_{1}^{3}=q x$ and $A=0$. This corresponds to an intersecting brane configuration with slope $q$. Considering this background solution a fluctuation analysis was done in [33] to analyze the spectrum of the theory. To review this 
analysis we first write down the relevant bosonic part of the action (A.13).

$$
S_{1+1}^{1}=\frac{1}{g^{2}} \operatorname{tr} \int d^{2} x\left[-\frac{1}{2} F_{\mu \nu} F^{\mu \nu}+D_{\mu} \Phi_{I} D^{\mu} \Phi_{I}+\frac{1}{2}\left[\Phi_{I}, \Phi_{J}\right]^{2}\right] .
$$

The Bosonic Lagrangian up to the quadratic order in fluctuations separates into various decoupled sets. In the $A_{0}^{a}=0(a=1,2,3)$ gauge we write the decoupled parts separately below.

$$
\begin{aligned}
\mathcal{L}\left(A_{x}^{2}, \Phi_{1}^{1}\right)= & -\frac{1}{2} A_{x}^{2} \partial_{0}^{2} A_{x}^{2}-\frac{1}{2} \Phi_{1}^{1} \partial_{0}^{2} \Phi_{1}^{1}+\frac{1}{2} \Phi_{1}^{1} \partial_{x}^{2} \Phi_{1}^{1}-q^{2} x^{2} \frac{1}{2}\left(A_{x}^{2}\right)^{2} \\
& +q A_{x}^{2} \Phi_{1}^{1}-q x \partial_{x} \Phi_{1}^{1} A_{x}^{2} . \\
\mathcal{L}\left(A_{x}^{1}, \Phi_{1}^{2}\right)= & -\frac{1}{2} A_{x}^{1} \partial_{0}^{2} A_{x}^{1}-\frac{1}{2} \Phi_{1}^{2} \partial_{0}^{2} \Phi_{1}^{2}+\frac{1}{2} \Phi_{1}^{2} \partial_{x}^{2} \Phi_{1}^{2}-q^{2} x^{2} \frac{1}{2}\left(A_{x}^{1}\right)^{2} \\
& -q A_{x}^{1} \Phi_{1}^{2}+q x \partial_{x} \Phi_{1}^{2} A_{x}^{1} \\
\mathcal{L}\left(\Phi_{I}, A_{x}^{3}\right)= & -\frac{1}{2} \Phi_{I}^{a} \partial_{0}^{2} \Phi_{I}^{a}+\frac{1}{2} \Phi_{I}^{a} \partial_{x}^{2} \Phi_{I}^{a}-\frac{1}{2} q^{2} x^{2}\left(\Phi_{I}^{1}\right)^{2}-\frac{1}{2} q^{2} x^{2}\left(\Phi_{I}^{2}\right)^{2} \\
& -\frac{1}{2} A_{x}^{3} \partial_{0}^{2} A_{x}^{3} \quad(\text { for all } I \neq 1) .
\end{aligned}
$$

We thus have various decoupled sets of equations at this quadratic order. The solutions of these equations give the wave functions corresponding to the normal modes. The first two terms $\mathcal{L}\left(A_{x}^{2}, \Phi_{1}^{1}\right)$ and $\mathcal{L}\left(A_{x}^{1}, \Phi_{1}^{2}\right)$ implies that we have a two coupled sets of equations for $\left(A_{x}^{2}, \Phi_{1}^{1}\right)$ and $\left(A_{x}^{1}, \Phi_{1}^{2}\right)$.

To compute eigenfunctions and the spectrum we first consider the equations for $\left(A_{x}^{2}, \Phi_{1}^{1}\right)$ fields. The eigenvalue equation for the spatial part is,

$$
\left(\begin{array}{cc}
m^{2}-q^{2} x^{2}-q x \partial_{x}+q \\
2 q+q x \partial_{x} & m^{2}+\partial_{x}^{2}
\end{array}\right)\left(\begin{array}{c}
A_{x}^{2} \\
\Phi_{1}^{1}
\end{array}\right)=0
$$

where $m^{2}$ is the eigenvalue. Eliminating, $A_{x}^{2}$ from the above equations gives the following equation for $\Phi_{1}^{1}$,

$$
\partial_{x}^{2} \Phi_{1}^{1}+[P(x)-Q(x)] \Phi_{1}^{1}-x P(x) \partial_{x} \Phi_{1}^{1}=0
$$

where,

$$
P(x)=\frac{2 q^{2}}{Q(x)} ; \quad Q(x)=q^{2} x^{2}-m^{2}
$$

The asymptotic $(x \rightarrow \infty)$ form of the equation (2.6) is,

$$
\left[\partial_{x}^{2}+m^{2}-q^{2} x^{2}\right] \Phi_{1}^{1}=0
$$

which is the Schroedinger's equation for a harmonic oscillator. The ground state wave function is $e^{-q x^{2} / 2}$. Thus writing,

$$
A_{x}^{2}=e^{-q x^{2} / 2} A ; \quad \Phi_{1}^{1}=e^{-q x^{2} / 2} \phi
$$


we get the following equations,

$$
\begin{aligned}
\left(m^{2}-q^{2} x^{2}\right) A+\left(q+q^{2} x^{2}-q x \partial_{x}\right) \phi & =0 \\
\left(-q^{2} x^{2}+q x \partial_{x}+2 q\right) A+\left(m^{2}-q+q^{2} x^{2}-2 q x \partial_{x}+\partial_{x}^{2}\right) \phi & =0 .
\end{aligned}
$$

Now assuming series solution of the form,

$$
A=\sum_{k} a_{k}(x \sqrt{q})^{k} ; \quad \phi=\sum_{k} b_{k}(x \sqrt{q})^{k}
$$

we get the following recursion relations,

$$
\frac{\left[(2 k-1)-\frac{m^{2}}{q}\right]}{\left[k-\frac{m^{2}}{q}\right]} b_{k}-\frac{(k+1)(k+2)}{\left[(k+2)-\frac{m^{2}}{q}\right]} b_{k+2}=0
$$

and

$$
a_{k}=b_{k}-\frac{(k+1)(k+2)}{\left[(k+2)-\frac{m^{2}}{q}\right]} b_{k+2} .
$$

The quantization condition on $m^{2}$ is obtained by demanding that the series (2.12) terminates for some value of $k$ that is $n$. This implies that the numerator of the first term of (2.13) vanishes for this value of $k$. We thus have the spectrum given by $m^{2}=$ $(2 n-1) q$. The lowest mode of mass spectrum given by $n=0$ is tachyonic. To solve for the eigenfunctions we simply need to compute the various coefficients $\left(a_{k}, b_{k}\right)$ using the recursion relations.

For $n=0,2,4 \cdots$,

$$
\begin{aligned}
a_{k} & =\frac{(-1)^{k / 2} 2^{k / 2}}{(2 n-1) k !} n(n-2) \cdots(n-k+2)(k-1) \\
b_{k} & =\frac{(-1)^{k / 2} 2^{k / 2}}{(2 n-1) k !} n(n-2) \cdots(n-k+2)(2 n-k-1) .
\end{aligned}
$$

For $n=3,5,7 \cdots$,

$$
\begin{aligned}
& a_{k}=\frac{(-1)^{(k-1) / 2} 2^{(k-1) / 2}}{(2 n-1) k !}(n-1)(n-3) \cdots(n-k+2)(k-1) \\
& b_{k}=\frac{(-1)^{(k-1) / 2} 2^{(k-1) / 2}}{2(n-1) k !}(n-1)(n-3) \cdots(n-k+2)(2 n-k-1) .
\end{aligned}
$$

Putting these back in (2.12), the eigenfunctions are,

$$
A_{n}=\sum_{k \leq n} a_{k}(x \sqrt{q})^{k} ; \quad \phi_{n}=\sum_{k \leq n} b_{k}(x \sqrt{q})^{k} .
$$

The normalized eigenfunctions $\left\{A_{n}(x), \phi_{n}(x)\right\}$ for both odd and even $n$ can be combined into the following expressions

$$
\begin{aligned}
& A_{n}(x)=\mathcal{N}(n) e^{-q x^{2} / 2}\left(H_{n}(\sqrt{q} x)+2 n H_{n-2}(\sqrt{q} x)\right) \\
& \phi_{n}(x)=\mathcal{N}(n) e^{-q x^{2} / 2}\left(H_{n}(\sqrt{q} x)-2 n H_{n-2}(\sqrt{q} x)\right)
\end{aligned}
$$


where $H_{n}(\sqrt{q} x)$ are Hermite polynomials and the normalization,

$$
\mathcal{N}(n)=1 / \sqrt{\sqrt{\pi} 2^{n}\left(4 n^{2}-2 n\right)(n-2) !} .
$$

Let us define,

$$
\zeta_{n}(x)=\left(\begin{array}{l}
A_{n}(x) \\
\phi_{n}(x)
\end{array}\right) .
$$

$\zeta_{n}(x)$ then satisfies the orthogonality condition

$$
\sqrt{q} \int d x \zeta_{n}^{\dagger}(x) \zeta_{n^{\prime}}(x)=\delta_{n, n^{\prime}}
$$

There is also a set of infinitely many degenerate eigenfunctions with $m_{n}^{2}=0$. The normalized eigenfunctions for the zero eigenvalues can also be written in terms of Hermite polynomials as

$$
\begin{aligned}
\tilde{A}_{n}(x) & =\tilde{\mathcal{N}}(n) e^{-q x^{2} / 2}\left(H_{n}(\sqrt{q} x)-2(n-1) H_{n-2}(\sqrt{q} x)\right) \\
\tilde{\phi}_{n}(x) & =\tilde{\mathcal{N}}(n) e^{-q x^{2} / 2}\left(H_{n}(\sqrt{q} x)+2(n-1) H_{n-2}(\sqrt{q} x)\right)
\end{aligned}
$$

where the normalization, $\tilde{\mathcal{N}}(n)=1 / \sqrt{\sqrt{\pi} 2^{n}(4 n-2)(n-1) !}$. We define a different set,

$$
\tilde{\zeta}_{n}(x)=\left(\begin{array}{c}
\tilde{A}_{n}(x) \\
\tilde{\phi}_{n}(x)
\end{array}\right) .
$$

$\tilde{\zeta}_{n}(x)$ again satisfies the orthogonality condition as (2.23). So that,

$$
\sqrt{q} \int d x \tilde{\zeta}_{n}^{\dagger}(x) \tilde{\zeta}_{n^{\prime}}(x)=\delta_{n, n^{\prime}}
$$

Along with this we also have,

$$
\sqrt{q} \int d x \zeta_{n}^{\dagger}(x) \tilde{\zeta}_{n^{\prime}}(x)=0 \text { for all } n \text { and } n^{\prime} .
$$

Unlike the non-zero eigenvalue sector, in the zero eigenvalue sector we have normalizable eigenfunction for $n=1$, which is simply $H_{1}(\sqrt{q} x)$. There is however no normalizable eigenfunctions for $n=0$ in this sector. The spectrum for $m_{n}^{2}=0$ is completely degenerate. Henceforth in this paper we shall refer the eigenfunctions for $m_{n}^{2}=0$ as the "zero-eigenfunctions".

From the equations of motion for $\left(A_{x}^{1}, \Phi_{1}^{2}\right)$ obtained from (2.3), their eigenfunctions are simply $\left(-A_{n}(x), \phi_{n}(x)\right)$, and $\left(-\tilde{A}_{n}(x), \tilde{\phi}_{n}(x)\right)$ for $m_{n}^{2}=(2 n-1) q$ and $m_{n}^{2}=0$ respectively. There is thus a two fold degeneracy for this spectrum of the theory.

The term $\mathcal{L}\left(\Phi_{I}, A_{x}^{3}\right)$ gives decoupled equations for $\Phi_{I}^{a}$ for each value of $I \neq 1$ and the gauge index $a$ and another equation for $A_{x}^{3}$ alone.The equation of motion for $\Phi_{I}^{1}$ is

$$
\left(-\partial_{0}^{2}+\partial_{x}^{2}-q^{2} x^{2}\right) \Phi_{I}^{1}=0 .
$$


The same equation for $\Phi_{I}^{2}$. The spatial part of the equation is the wave function equation for a Harmonic oscillator. The time independent eigenfunctions will thus be given by $\mathcal{N}^{\prime}(n) e^{-q x^{2} / 2} H_{n}(\sqrt{q} x)$ where $H_{n}(\sqrt{q} x)$ are Hermite polynomials. The normalization $\mathcal{N}^{\prime}(n)=1 / \sqrt{\sqrt{\pi} 2^{n} n !}$. The corresponding eigenvalues are $\gamma_{n}=(2 n+1) q$. The equations of motion for $\Phi_{I}^{3}$ and $A_{1}^{3}$ are,

$$
\left(-\partial_{0}^{2}+\partial_{x}^{2}\right) \Phi_{I}^{3}=0 ; \quad \partial_{0}^{2} A_{x}^{3}=0 .
$$

This means that the time independent part of $\Phi_{I}^{3}$ is just a plane wave $e^{i l x}$. Tables 1 and 2 in appendix B summarizes the various dimensionfull parameters, normalizations and the eigenfunctions.

At this point we should note that the only tachyons that arise in the spectrum are those as discussed above. There are no other tachyons. The presence of the tachyon signals an instability. As noted in [1] and the introduction, this instability corresponds to the onset of superconducting phase transition of the baryons in the boundary theory. In the brane picture, the tachyon condenses and at the end of the process we are left with a smoothened out brane configuration [33]. In the following sections we would like to study the quantum theory at finite temperature. The main aim is to find the critical temperature at which the tachyonic instability vanishes.

\section{Finite temperature analysis with one scalar: warm up exercise}

In this section we first study a simplified model consisting of only one adjoint scalar. The purpose of this section is to outline the basic idea involved in the computation of the mass-squared corrections of the tachyon due to finite temperature effects. With only one scalar (namely $\Phi_{1}^{1}$ ) we have the equations resulting from (2.2), up to the quadratic order. By doing a one-loop integral over the fluctuations we will find an effective action for the tachyonic mode. The coefficient of the quadratic part of the effective action gives the mass-squared of the tachyon as function of the temperature. We thus start by doing a fluctuation analysis with the doublet of fields $\left(\Phi_{1}^{1}, A_{x}^{2}\right)$ fields.

$$
A_{x}^{2}=A_{B}+\delta A \quad \Phi_{1}^{1}=\Phi_{B}+\delta \Phi .
$$

To do a finite temperature analysis, we define the Euclidean coordinate $\tau=i t$. $\tau$ is periodic with period $\beta$, so that the integration limits over $\tau$ are from 0 to $\beta$.

We now denote the background field and the fluctuations as,

$$
\zeta(x, \tau)=\left(\begin{array}{l}
A_{B}(x, \tau) \\
\Phi_{B}(x, \tau)
\end{array}\right) \quad, \quad \delta \zeta(x, \tau)=\left(\begin{array}{l}
\delta A(x, \tau) \\
\delta \Phi(x, \tau)
\end{array}\right) .
$$

The quadratic background part of the action can be written as,

$$
S_{B}=\frac{1}{2 g^{2}} \int d \tau d x \zeta^{\dagger}(x, \tau) \mathcal{O}_{0}(x, \tau) \zeta(x, \tau)
$$

where

$$
\mathcal{O}_{0}(x, \tau)=\left(\begin{array}{cc}
\partial_{\tau}^{2}-q^{2} x^{2} & -q x \partial_{x}+q \\
2 q+q x \partial_{x} & \partial_{\tau}^{2}+\partial_{x}^{2}
\end{array}\right)
$$


The mode expansions for the background fields is,

$$
\begin{aligned}
\zeta(x, \tau) & =N^{1 / 2} \sum_{w, k}\left(C_{w, k} \zeta_{k}(x)+\tilde{C}_{w, k} \tilde{\zeta}_{k}(x)\right) e^{i \omega_{w} \tau} \\
& =N^{1 / 2} \sum_{w, k}\left(C_{w, k}\left(\begin{array}{c}
A_{k}(x) \\
\phi_{k}(x)
\end{array}\right)+\tilde{C}_{w, k}\left(\begin{array}{c}
\tilde{A}_{k}(x) \\
\tilde{\phi}_{k}(x)
\end{array}\right)\right) e^{i \omega_{w} \tau}
\end{aligned}
$$

Where $\omega_{w}=2 \pi w / \beta$. The normalization constant $N$ is equal to $\sqrt{q} / \beta$. $\zeta_{k}(x)$ and $\tilde{\zeta}_{k}(x)$ are defined in (2.22) ans (2.24) respectively. The corresponding eigenvalues are $-\lambda_{k}=$ $-(2 k-1) q$ of the first set of eigenfunctions and $\tilde{\lambda}_{k}=0$ (for all $k$ ) of the second set. The reality of $\zeta(x, \tau)$ means that $C_{-w, k}=C_{w, k}^{*}$ and $\tilde{C}_{-w, k}=\tilde{C}_{w, k}^{*}$.

With these observations, and using the orthogonality properties (2.23), (2.27) and (2.28) the quadratic background part of the action can then be written as,

$$
S_{B}=-\frac{1}{2 g^{2}} \sum_{w, k}\left(\left|C_{w, k}\right|^{2}\left(\omega_{w}^{2}+\lambda_{k}\right)+\left|\tilde{C}_{w, k}\right|^{2} \omega_{w}^{2}\right)
$$

The mass spectrum now consists of a tower of tachyons of mass-squared $\left(\omega_{w}^{2}-q\right) / g^{2}$. Of these the zero mode, $w=0$ has the lowest value of mass $^{2}$.

We now study the fluctuations about the above background. The part of the Lagrangian containing the fluctuations is given by,

$$
S_{\delta}=\frac{1}{2 g^{2}} \int d \tau d x \delta \zeta^{\dagger}(x, \tau) \mathcal{O}_{\delta}(x, \tau) \delta \zeta(x, \tau)
$$

where the operator $\mathcal{O}_{\delta}(x, \tau)$ is,

$$
\begin{aligned}
\mathcal{O}_{\delta}(x, \tau) & =\mathcal{O}_{0}(x, \tau)+\mathcal{O}_{B}(x, \tau) \\
& =\left(\begin{array}{cc}
\partial_{\tau}^{2}-q^{2} x^{2}-\Phi_{B}^{2}(x, \tau) & -q x \partial_{x}+q-2 A_{B}(x, \tau) \Phi_{B}(x, \tau) \\
2 q+q x \partial_{x}-2 A_{B}(x, \tau) \Phi_{B}(x, \tau) & \partial_{\tau}^{2}+\partial_{x}^{2}-A_{B}^{2}(x, \tau)
\end{array}\right) .
\end{aligned}
$$

There will also be terms linear in the fluctuations. However since these terms do not contribute to the $1 P I$ effective action, we have dropped them here.

The mode expansions for the fluctuations is,

$$
\begin{aligned}
\delta \zeta(x, \tau) & =N^{1 / 2} \sum_{m, n}\left(D_{m, n} \delta \zeta_{n}(x)+\tilde{D}_{m, n} \delta \tilde{\zeta}_{n}(x)\right) e^{i \omega_{m} \tau} \\
& =N^{1 / 2} \sum_{m, n}\left(D_{m, n}\left(\begin{array}{c}
\delta A_{n}(x) \\
\delta \phi_{n}(x)
\end{array}\right)+\tilde{D}_{m, n}\left(\begin{array}{c}
\delta \tilde{A}_{n}(x) \\
\delta \tilde{\phi}_{n}(x)
\end{array}\right)\right) e^{i \omega_{m} \tau}
\end{aligned}
$$

where $\delta \zeta_{n}(x)$ and $\delta \tilde{\zeta}_{n}(x)$ are now eigenfunctions of the $\tau$-independent part of the operator $\mathcal{O}_{\delta}(x, \tau)$. Let us assume that the corresponding eigenvalues are $-\Lambda_{n}$ and $-\tilde{\Lambda}_{n}$ respectively. Again since $\delta \zeta(x, \tau)$ is real, $D_{-m, n}=D_{m, n}^{*}$ and $\tilde{D}_{-m, n}=\tilde{D}_{m, n}^{*}$. The partition function for 
the fluctuations is thus,

$$
\begin{aligned}
Z(\beta, q) & =\int \mathcal{D}\left[D_{m, n}\right]\left[\tilde{D}_{m, n}\right] e^{S_{\delta}} \\
& =\int \mathcal{D}\left[D_{m, n}\right]\left[\tilde{D}_{m, n}\right] e^{-\frac{1}{2 g^{2}} \sum_{m, n}\left[\left|D_{m, n}\right|^{2}\left(\omega_{m}^{2}+\Lambda_{n}\right)+\left|\tilde{D}_{m, n}\right|^{2}\left(\omega_{m}^{2}+\tilde{\Lambda}_{n}\right)\right]} \\
& =\prod_{m, n \neq 0}\left[\frac{1}{\left(2 \pi g^{2}\right)^{2}}\left(\omega_{m}^{2}+\Lambda_{n}\right)\left(\omega_{m}^{2}+\tilde{\Lambda}_{n}\right)\right]^{-1 / 2} .
\end{aligned}
$$

The eigenvalues $\Lambda_{n}$ and $\tilde{\Lambda}_{n}$ are yet to be determined, for which we use perturbation theory by assuming that the background field modes are small. We already know the time independent eigenfunctions and the corresponding eigenvalues for the operator $\mathcal{O}_{0}$. We can now treat the background fields in (3.8) as perturbations and find the corrections. The background fields can be expanded in terms of the $C_{w, k}$ modes. Since we are only interested in the quadratic contribution in $C_{w, k}$ 's, we do not need beyond the leading correction. This is because the perturbation matrix $\mathcal{O}_{B}$ contains two powers of background fields giving rise to terms quadratic in the $C_{w, k}$ modes.

$$
\begin{array}{rlrlrl}
\Lambda_{n} & =\Lambda_{n}^{(0)}+\Lambda_{n}^{(1)}+\ldots & \text { with } & \Lambda_{n}^{(0)} & =\lambda_{n}=(2 n-1) q \\
\tilde{\Lambda}_{n} & =\tilde{\Lambda}_{n}^{(0)}+\tilde{\Lambda}_{n}^{(1)}+\ldots & \text { with } & \tilde{\Lambda}_{n}^{(0)}=\tilde{\lambda}_{n}=0
\end{array}
$$

and,

$$
\begin{aligned}
& \delta \zeta_{n}(x)=\delta \zeta_{n}^{(0)}(x)+\delta \zeta_{n}^{(1)}(x)+\ldots \quad \text { with } \quad \delta \zeta_{n}^{(0)}(x)=\zeta_{n}(x) \\
& \delta \tilde{\zeta}_{n}(x)=\delta \tilde{\zeta}_{n}^{(0)}(x)+\delta \tilde{\zeta}_{n}^{(1)}(x)+\ldots \quad \text { with } \quad \delta \tilde{\zeta}_{n}^{(0)}(x)=\tilde{\zeta}_{n}(x)
\end{aligned}
$$

so that,

$$
\begin{aligned}
& \Lambda_{n}^{(1)}=-\int d x d \tau \delta \zeta_{n}^{(0) \dagger}(x) \mathcal{O}_{B}(x, \tau) \delta \zeta_{n}^{(0)}(x) \\
& \tilde{\Lambda}_{n}^{(1)}=-\int d x d \tau \delta \tilde{\zeta}_{n}^{(0) \dagger}(x) \mathcal{O}_{B}(x, \tau) \delta \tilde{\zeta}_{n}^{(0)}(x)
\end{aligned}
$$

with this,

$$
\begin{aligned}
\log Z(\beta, q) & =-\frac{1}{2} \sum_{m, n \neq 0}\left[\log \left(\frac{\omega_{m}^{2}+(2 n-1) q+\Lambda_{n}^{(1)}}{2 \pi g^{2}}\right)+\log \left(\frac{\omega_{m}^{2}+\tilde{\Lambda}_{n}^{(1)}}{2 \pi g^{2}}\right)\right] \\
& =-\frac{1}{2} \sum_{m, n \neq 0}\left[\log \left(1+\frac{\Lambda_{n}^{(1)} \beta^{2}}{(2 \pi m)^{2}+(2 n-1) q \beta^{2}}\right)+\log \left(1+\frac{\tilde{\Lambda}_{n}^{(1)} \beta^{2}}{(2 \pi m)^{2}}\right)\right]
\end{aligned}
$$

where in the last line we have omitted the field independent terms. For small value of $\Lambda_{n}^{(1)}$ the leading term which gives the quadratic correction to the effective action of the background $C_{w, k}$ fields is,

$$
\log Z(\beta, q)=-\frac{1}{2} \sum_{m, n \neq 0}\left[\frac{\Lambda_{n}^{(1)} \beta^{2}}{(2 \pi m)^{2}+(2 n-1) q \beta^{2}}+\frac{\tilde{\Lambda}_{n}^{(1)} \beta^{2}}{(2 \pi m)^{2}}\right] .
$$


To find the form of the effective action due to the perturbations we first compute $\Lambda_{n}^{(1)}$ given in equation (3.15). The expression for $\Lambda_{n}^{(1)}$ after putting in the mode expansions for the background $\Phi_{B}$ and $A_{B}$ fields reads,

$$
\begin{aligned}
\Lambda_{n}^{(1)}=\sum_{w, k, k^{\prime}}\left[C_{w, k} C_{w, k^{\prime}}^{*}\right. & \left.F_{1}\left(k, k^{\prime}, n, n\right)+\tilde{C}_{w, k} \tilde{C}_{w, k^{\prime}}^{*} F_{1}^{\prime}\left(k, k^{\prime}, n, n\right)+2 C_{w, k} \tilde{C}_{w, k^{\prime}}^{*} F_{1}^{\prime \prime}\left(k, k^{\prime}, n, n\right)\right] \\
F_{1}\left(k, k^{\prime}, n, n\right)= & \sqrt{q} \int d x\left[\phi_{k}(x) \phi_{k^{\prime}}(x) A_{n}(x) A_{n}(x)+2 A_{k} \phi_{k^{\prime}}(x) \phi_{n}(x) A_{n}(x)\right. \\
& \left.+2 A_{k^{\prime}} \phi_{k}(x) \phi_{n}(x) A_{n}(x)+A_{k}(x) A_{k^{\prime}}(x) \phi_{n}(x) \phi_{n}(x)\right] \\
F_{1}^{\prime}\left(k, k^{\prime}, n, n\right)= & \sqrt{q} \int d x\left[\tilde{\phi}_{k}(x) \tilde{\phi}_{k^{\prime}}(x) A_{n}(x) A_{n}(x)+2 \tilde{A}_{k} \tilde{\phi}_{k^{\prime}}(x) \phi_{n}(x) A_{n}(x)\right. \\
& \left.+2 \tilde{A}_{k^{\prime}} \tilde{\phi}_{k}(x) \phi_{n}(x) A_{n}(x)+\tilde{A}_{k}(x) \tilde{A}_{k^{\prime}}(x) \phi_{n}(x) \phi_{n}(x)\right] \\
F_{1}^{\prime \prime}\left(k, k^{\prime}, n, n\right)= & \sqrt{q} \int d x\left[\phi_{k}(x) \tilde{\phi}_{k^{\prime}}(x) A_{n}(x) A_{n}(x)+2 A_{k} \tilde{\phi}_{k^{\prime}}(x) \phi_{n}(x) A_{n}(x)\right. \\
& \left.+2 \tilde{A}_{k^{\prime}} \phi_{k}(x) \phi_{n}(x) A_{n}(x)+A_{k}(x) \tilde{A}_{k^{\prime}}(x) \phi_{n}(x) \phi_{n}(x)\right] .
\end{aligned}
$$

Similarly expanding $\tilde{\Lambda}_{n}^{(1)}$ given in (3.16) gives,

$$
\begin{aligned}
\tilde{\Lambda}_{n}^{(1)}=\sum_{w, k, k^{\prime}}\left[C_{w, k} C_{w, k^{\prime}}^{*}\right. & \left.\tilde{F}_{1}\left(k, k^{\prime}, n, n\right)+\tilde{C}_{w, k} \tilde{C}_{w, k^{\prime}}^{*} \tilde{F}_{1}^{\prime}\left(k, k^{\prime}, n, n\right)+2 C_{w, k} \tilde{C}_{w, k^{\prime}}^{*} \tilde{F}_{1}^{\prime \prime}\left(k, k^{\prime}, n, n\right)\right] \\
\tilde{F}_{1}\left(k, k^{\prime}, n, n\right)= & \sqrt{q} \int d x\left[\phi_{k}(x) \phi_{k^{\prime}}(x) \tilde{A}_{n}(x) \tilde{A}_{n}(x)+2 A_{k} \phi_{k^{\prime}}(x) \tilde{\phi}_{n}(x) \tilde{A}_{n}(x)\right. \\
& \left.+2 A_{k^{\prime}} \phi_{k}(x) \tilde{\phi}_{n}(x) \tilde{A}_{n}(x)+A_{k}(x) A_{k^{\prime}}(x) \tilde{\phi}_{n}(x) \tilde{\phi}_{n}(x)\right] \\
\tilde{F}_{1}^{\prime}\left(k, k^{\prime}, n, n\right)= & \sqrt{q} \int d x\left[\tilde{\phi}_{k}(x) \tilde{\phi}_{k^{\prime}}(x) \tilde{A}_{n}(x) \tilde{A}_{n}(x)+2 \tilde{A}_{k} \tilde{\phi}_{k^{\prime}}(x) \tilde{\phi}_{n}(x) \tilde{A}_{n}(x)\right. \\
& \left.+2 \tilde{A}_{k^{\prime}} \tilde{\phi}_{k}(x) \tilde{\phi}_{n}(x) \tilde{A}_{n}(x)+\tilde{A}_{k}(x) \tilde{A}_{k^{\prime}}(x) \tilde{\phi}_{n}(x) \tilde{\phi}_{n}(x)\right] \\
\tilde{F}_{1}^{\prime \prime}\left(k, k^{\prime}, n, n\right)= & \sqrt{q} \int d x\left[\phi_{k}(x) \tilde{\phi}_{k^{\prime}}(x) \tilde{A}_{n}(x) \tilde{A}_{n}(x)+2 A_{k} \tilde{\phi}_{k^{\prime}}(x) \tilde{\phi}_{n}(x) \tilde{A}_{n}(x)\right. \\
& \left.+2 \tilde{A}_{k^{\prime}} \phi_{k}(x) \tilde{\phi}_{n}(x) \tilde{A}_{n}(x)+A_{k}(x) \tilde{A}_{k^{\prime}}(x) \tilde{\phi}_{n}(x) \tilde{\phi}_{n}(x)\right] .
\end{aligned}
$$

Putting these expansions (3.19) and (3.23) in (3.18) we now collect terms containing two $C_{w, k}$ fields, two $\tilde{C}_{w, k}$ or one $C_{w, k}$ and one $\tilde{C}_{w, k}$ modes separately. A general expression for (3.18) finally is,

$$
\begin{aligned}
\log Z(\beta, q)=-\sum_{w, k, k^{\prime}}[ & C_{w, k} C_{w, k^{\prime}}^{*} \Sigma^{2}\left(k, k^{\prime}, \beta, q\right)+\tilde{C}_{w, k} \tilde{C}_{w, k^{\prime}}^{*} \tilde{\Sigma}^{2}\left(k, k^{\prime}, \beta, q\right) \\
& \left.+2 C_{w, k} \tilde{C}_{w, k^{\prime}}^{*} \Sigma^{\prime 2}\left(k, k^{\prime}, \beta, q\right)\right] .
\end{aligned}
$$


The coefficient of the $C_{w, k} C_{w, k^{\prime}}^{*}$ term, $\Sigma^{2}\left(k, k^{\prime}, \beta, q\right)$ is given by,

$$
\Sigma^{2}\left(k, k^{\prime}, \beta, q\right)=\frac{1}{2} \sqrt{q} \beta \sum_{m, n \neq 0}\left[\frac{F_{1}\left(k, k^{\prime}, n, n\right)}{(2 \pi m)^{2}+(2 n-1) q \beta^{2}}+\frac{\tilde{F}_{1}\left(k, k^{\prime}, n, n\right)}{(2 \pi m)^{2}}\right] .
$$

This is the one-loop correction to the two point amplitude for the $C_{w, k}$ modes. Similarly,

$$
\tilde{\Sigma}^{2}\left(k, k^{\prime}, \beta, q\right)=\frac{1}{2} \sqrt{q} \beta \sum_{m, n \neq 0}\left[\frac{F_{1}^{\prime}\left(k, k^{\prime}, n, n\right)}{(2 \pi m)^{2}+(2 n-1) q \beta^{2}}+\frac{\tilde{F}_{1}^{\prime}\left(k, k^{\prime}, n, n\right)}{(2 \pi m)^{2}}\right]
$$

and

$$
\Sigma^{\prime 2}\left(k, k^{\prime}, \beta, q\right)=\frac{1}{2} \sqrt{q} \beta \sum_{m, n \neq 0}\left[\frac{F_{1}^{\prime \prime}\left(k, k^{\prime}, n, n\right)}{(2 \pi m)^{2}+(2 n-1) q \beta^{2}}+\frac{\tilde{F}_{1}^{\prime \prime}\left(k, k^{\prime}, n, n\right)}{(2 \pi m)^{2}}\right] .
$$

The fields $C_{w, k}$ and $\tilde{C}_{w, k}$ for different $k$ and same $w$ are all coupled to each other at the quadratic order. This is due to the broken translational along the $x$ direction. To compute the mass-squared correction of any of the modes we should compute the mass matrix. However this matrix is infinite dimensional. We will be interested in the masssquared corrections of the mode $C_{0,0}$. This we do numerically. In this paper for numerical simplicity we just compute the correction term $\Sigma^{2}(0,0, \beta, q)$, reserving a more detailed numerical analysis for the future.

Now, after doing the sum over $m$ in the first term of (3.28),

$$
\begin{aligned}
\Sigma^{2}\left(k, k^{\prime}, \beta, q\right) & =\frac{1}{2} \sum_{n \neq 0}\left[\frac{F_{1}\left(k, k^{\prime}, n, n\right)}{\sqrt{(2 n-1)}}\left(\frac{1}{2}+\frac{1}{e^{\sqrt{(2 n-1) q} \beta}-1}\right)+\frac{\tilde{F}_{1}\left(k, k^{\prime}, n, n\right)}{(2 \pi m)^{2}}\right] \\
& =\Sigma_{v a c}^{2}+\Sigma_{\beta}^{2} .
\end{aligned}
$$

$\Sigma_{v a c}^{2}$ is the temperature independent piece. This term is potentially ultraviolet divergent. In the following sections we shall consider the the theory on the intersecting $D 1$ branes. This theory is obtained from a finite $\mathcal{N}=8 \mathrm{SYM}$ in two dimensions by giving an expectation value to one of the scalars $\Phi_{1}^{3}=q x$. Supersymmetry in the intersecting $D 1$ brane theory is completely broken by the background. However the action is supersymmetric and finiteness of the $\mathcal{N}=8$ theory implies that the the theory on the intersecting $D 1$ branes must also be ultraviolet finite. We will see that for the two point functions computed later the ultraviolet divergences cancel between the contributions from the boson and the fermion loops.

There is also an infrared divergence in (3.31) that comes from the second term for $m=0$. The IR divergence is due to the massless $\tilde{D}_{m, n}$ modes in the loop. To treat these divergences we shall follow the procedure outlined in the introduction.

A similar computation leading to (3.28), (3.29) and (3.30) can also be performed as follows. Expanding both the background and the fluctuation fields using same basis functions 
that are the eigenfunctions of $\mathcal{O}_{0}$ i.e. to the lowest order the fluctuation wave functions, $\delta A_{n}(x)=A_{n}(x), \delta \phi_{n}(x)=\phi_{n}(x), \delta \tilde{A}_{n}(x)=\tilde{A}_{n}(x)$ and $\delta \tilde{\phi}_{n}(x)=\tilde{\phi}_{n}(x)$,

$$
\begin{aligned}
S= & S_{B}+S_{\delta} \\
= & -\frac{1}{2 g^{2}} \sum_{w, k}\left(\left|C_{w, k}\right|^{2}\left(\omega_{w}^{2}+\lambda_{k}\right)+\left|\tilde{C}_{w, k}\right|^{2} \omega_{w}^{2}\right)-\frac{1}{2 g^{2}} \sum_{m, n}\left(\left|D_{m, n}\right|^{2}\left(\omega_{m}^{2}+\lambda_{n}\right)+\left|\tilde{D}_{m, n}\right|^{2} \omega_{m}^{2}\right) \\
& +I+\text { background fields of quartic order }
\end{aligned}
$$

where the interaction term $I$, is

$$
\begin{aligned}
I= & -\frac{N}{2 g^{2}} \sum_{m, m^{\prime}, n, n^{\prime}} \sum_{w, w^{\prime}, k, k^{\prime}}\left(C_{w, k} C_{w^{\prime}, k^{\prime}} D_{m, n} D_{m^{\prime}, n^{\prime}} F_{1}\left(k, k^{\prime}, n, n^{\prime}\right)\right. \\
& +C_{w, k} C_{w^{\prime}, k^{\prime}} \tilde{D}_{m, n} \tilde{D}_{m^{\prime}, n^{\prime}} \tilde{F}_{1}\left(k, k^{\prime}, n, n^{\prime}\right)+\tilde{C}_{w, k} \tilde{C}_{w^{\prime}, k^{\prime}} D_{m, n} D_{m^{\prime}, n^{\prime}} F^{\prime}{ }_{1}\left(k, k^{\prime}, n, n^{\prime}\right) \\
& +\tilde{C}_{w, k} \tilde{C}_{w^{\prime}, k^{\prime}} \tilde{D}_{m, n} \tilde{D}_{m^{\prime}, n^{\prime}} \tilde{F}^{\prime}{ }_{1}\left(k, k^{\prime}, n, n^{\prime}\right)+2 C_{w, k} \tilde{C}_{w^{\prime}, k^{\prime}} D_{m, n} D_{m^{\prime}, n^{\prime}} F^{\prime \prime}{ }_{1}\left(k, k^{\prime}, n, n^{\prime}\right) \\
& \left.+2 C_{w, k} \tilde{C}_{w^{\prime}, k^{\prime}} \tilde{D}_{m, n} \tilde{D}_{m^{\prime}, n^{\prime}} \tilde{F}^{\prime \prime}{ }_{1}\left(k, k^{\prime}, n, n^{\prime}\right)\right) \delta_{m+m^{\prime}+w+w^{\prime}, 0}
\end{aligned}
$$

where, $N=\sqrt{q} / \beta$. Here again we have dropped the terms linear in fluctuations as they do not contribute to the $1 P I$ effective action. The terms cubic in fluctuations have also not been included as they do not contribute at the one-loop order. The tree-level $D_{m, n}$ and $\tilde{D}_{m, n}$ propagators are then

$$
\left\langle D_{m, n} D_{m^{\prime}, n^{\prime}}\right\rangle=g^{2} \frac{\delta_{m,-m^{\prime}} \delta_{n, n^{\prime}}}{\left[\omega_{m}^{2}+\lambda_{n}\right]} ; \quad\left\langle\tilde{D}_{m, n} \tilde{D}_{m^{\prime}, n^{\prime}}\right\rangle=g^{2} \frac{\delta_{m,-m^{\prime}} \delta_{n, n^{\prime}}}{\omega_{m}^{2}}
$$

With this the one-loop two point amplitudes are same as equations (3.28), (3.29) and (3.30). Henceforth in the following sections we will denote both the background and the fluctuation modes as $C_{w, k}$.

\section{Intersecting D1 branes at finite temperature}

We have computed the effective mass-squared of the tachyons as function of temperature in section 3 for a simplified theory with only one scalar field and no fermions. The corrections are infrared divergent due to the presence of the tree-level massless modes in the loops. One way of dealing with the problem is by computing the mass-squared corrections to the tree-level massless modes $\tilde{C}_{w, k}$, at finite temperature at the one-loop level. With the mass-squared corrections, the tree-level massless modes become massive at one-loop level at finite temperature. The temperature-dependent effective mass-squared of the tree-level massless modes shift their propagator by $\omega_{m}^{-2} \rightarrow\left(\omega_{m}^{2}+m^{2}(\beta)\right)^{-1}$. Now we can use this shifted propagator to calculate the finite effective mass-squared of the tree-level tachyons at finite temperature.

The first amplitude in (3.31) has a purely temperature independent part and a purely temperature dependent part. The temperature dependent part is exponentially damped, hence finite even for large values of the momentum $n$. The temperature independent 
part however has a non-convergent sum over the momentum $n$ and hence give rise to Ultraviolet divergence. The problem of UV divergence can be dealt with by introducing suitable regularization scheme but this in turn renders the finite answer for the masssquared corrections regularization-dependent. Hence there is no unique answer for the effective mass-squared of the tachyon. This indicates that the calculation should be done in a framework where cancellation of UV divergences are possible. Hence we work in the framework of a originally supersymmetric theory and should consider the contributions from the bosonic as well as fermionic degrees of freedom. In this set-up the UV divergences are expected to cancel among the amplitudes containing bosons and fermions in the loop.

In this section we thus study the finite temperature effects for the full $D 1$ brane theory (A.13). As seen in section 2 an intersecting brane configuration with only one nonzero angle is given by the background solution $\Phi_{1}^{3}=q x$ and $A=0$. In the following we will study the tachyon mass-squared as a function of the temperature for the theory (A.13) including all the other bosonic fields and the fermions. We will compute contribution from Bosons and the Fermions towards the tachyon mass-squared correction separately below.

\subsection{Bosons}

In this section we compute the one-loop correction to the tree-level tachyon mass-squared due to the Bosons in the loop. To do this we must first write the mode expansions for the individual fields. The mode expansion for $\left(A_{x}^{2}, \Phi_{1}^{1}\right)$ is given in (3.5) in section 3 using the eigenfunctions that have been worked out in section 2 . The $\left(A_{x}^{1}, \Phi_{1}^{2}\right)$ fields satisfy the same mode expansion. The only distinction between this and the earlier mode expansion is the sign in front of the eigenfunctions $A_{k}(x)$. Thus

$$
\begin{aligned}
\zeta^{\prime}(x, \tau) & =N^{1 / 2} \sum_{w, k}\left(C_{w, k}^{\prime} \zeta_{k}(x) e^{i \omega_{w} \tau}+\tilde{C}_{w, k}^{\prime} \tilde{\zeta}_{k}(x) e^{i \omega_{w} \tau}\right) \\
& =N^{1 / 2} \sum_{w, k}\left(C_{1 w, k}^{\prime}\left(\begin{array}{c}
-A_{k}(x) \\
\phi_{k}(x)
\end{array}\right) e^{i \omega_{w} \tau}+\tilde{C}_{w, k}^{\prime}\left(\begin{array}{c}
-\tilde{A}_{k}(x) \\
\tilde{\phi}_{k}(x)
\end{array}\right) e^{i \omega_{w} \tau}\right)
\end{aligned}
$$

where $\zeta^{\prime}(x, \tau)=\left(\begin{array}{c}A_{x}^{1}(x, \tau) \\ \Phi_{1}^{2}(x, \tau)\end{array}\right)$.

Similarly since $\Phi_{I}^{1}$ and $\Phi_{I}^{2}(I \neq 1)$ are harmonic oscillators in the $x$ direction and $A_{x}^{3}$ and $\Phi_{I}^{3}$ (for all $I$ ) are just plane waves, we have the following mode expansions

$$
\begin{aligned}
& \Phi_{I}^{1}(x, \tau)=N^{1 / 2} \mathcal{N}^{\prime}(n) \sum_{m, n} \Phi_{I}^{1}(n, m) e^{-q x^{2} / 2} H_{n}(\sqrt{q} x) e^{i \omega_{m} \tau}, \quad\{I \neq 1\} \\
& \Phi_{I}^{3}(x, \tau)=\frac{N^{1 / 2}}{\sqrt{q}} \sum_{m} \int \frac{d l}{2 \pi} \Phi_{I}^{3}(l, m) e^{i\left(\omega_{m} \tau+l x\right)},\{I=1, \cdots, 8\} \\
& A_{x}^{3}(x, \tau)=\frac{N^{1 / 2}}{\sqrt{q}} \int \frac{d l}{2 \pi} \sum_{m} A_{x}^{3}(m, l) e^{i \omega_{m} \tau+i l x}
\end{aligned}
$$

where $H_{n}(\sqrt{q} x)$ are the Hermite polynomials and $e^{-q x^{2} / 2} H_{n}(\sqrt{q} x)$ are the harmonic oscillator wave functions with eigenvalue $\gamma_{n}=(2 n+1) q$. The normalization $\mathcal{N}^{\prime}(n)=$ $1 / \sqrt{\sqrt{\pi} 2^{n} n !}$. 
The propagators and the interaction vertices are listed in the appendix (C.1). With these we now write down the contribution to the one loop mass-squared corrections to the background fields. The bosonic contributions to the one loop mass-squared corrections at finite temperature can be collected in two groups, namely the ones coming from the four-point vertices and the ones coming from the three-point vertices.

The bosonic four-point vertices listed in (C.1) together with their corresponding propagators give,

$$
\begin{aligned}
\Sigma^{1}\left(w, w^{\prime}, k, k^{\prime}, \beta, q\right)=\frac{1}{2} N \sum_{m}\left[\sum_{n}\right. & \left(\frac{F_{1}\left(k, k^{\prime}, n, n\right)}{\omega_{m}^{2}+\lambda_{n}}+\frac{\tilde{F}_{1}\left(k, k^{\prime}, n, n\right)}{\omega_{m}^{2}}+\frac{7 F_{2}\left(k, k^{\prime}, n, n\right)}{\omega_{m}^{2}+\gamma_{n}}\right) \\
& +\int \frac{d l}{(2 \pi \sqrt{q})}\left(\frac{7 F_{2}^{\prime}\left(k, k^{\prime}, l,-l\right)}{\omega_{m}^{2}+l^{2}}+\frac{F_{3}^{\prime}\left(k, k^{\prime}, l,-l\right)}{\omega_{m}^{2}+l^{2}}\right) \\
& \left.+\int \frac{d l}{2 \pi \sqrt{q}} \frac{F_{3}\left(k, k^{\prime}, l,-l\right)}{\omega_{m}^{2}}\right] \delta_{w+w^{\prime}}
\end{aligned}
$$

The Feynman diagrams that constitute the correction (4.5) are given in figure 1. In (4.5) the first term represented by the Feynman diagram in figure 1(a) consists of the three-point vertex (C.6) and receives contributions from the $C_{m, n}$ modes with the propagators (C.1) while the second term whose Feynman diagram is given is figure 1(b) comes from the four-point vertex (C.10) and bears contributions from the massless modes $\tilde{C}_{m, n}$ in the loop with propagator (C.2). The third term with the Feynman diagram figure 1(c) comprises of the four-point vertex (C.8) having the seven massive fields $\Phi_{I}^{1,2}(m, n)$ for $I \neq 1$ with propagators given in (C.3). The fourth term in (4.5) is represented by the Feynman diagram figure $1(\mathrm{~d})$ and is the amplitude for the vertex (C.12) which comprise of the seven fields $\Phi_{I}^{3}$, for $I \neq 1$, with propagator (C.4). The fifth term have contributions from the fields $\Phi_{1}^{3}$ with propagators (C.4) and is depicted in the Feynman diagram figure 1(f) while the sixth term bears the massless gauge field $A_{x}^{3}(m, l)$ in the loop with propagator (C.5) and the relevant Feynman diagram given in figure 1(e). Similarly, the three-point bosonic vertices listed in (C.1) combined with their respective propagators constitute the one-loop bosonic mass-squared corrections at finite temperature, viz.

$$
\begin{aligned}
\Sigma^{2}\left(w, w^{\prime}, k, k^{\prime}, \beta, q\right)= & -\frac{1}{2} q N \sum_{m, n}\left[\int \frac{d l}{2 \pi \sqrt{q}} \frac{F_{4}(k, l, n) F_{4}^{*}\left(k^{\prime}, l, n\right)}{\left(\omega_{m}^{2}+\lambda_{n}\right) \omega_{m^{\prime}}^{2}}\right. \\
& +\int \frac{d l}{2 \pi \sqrt{q}} \frac{\tilde{F}_{4}(k, l, n) \tilde{F}_{4}^{*}\left(k^{\prime}, l, n\right)}{\omega_{m}^{2} \omega_{m^{\prime}}^{2}} \\
& +\int \frac{d l}{2 \pi \sqrt{q}}\left(\frac{7 F_{5}(k, l, n) F_{5}^{*}\left(k^{\prime},-l, n\right)}{\left(\omega_{m}^{2}+\gamma_{n}\right)\left(\omega_{m^{\prime}}^{2}+l^{2}\right)}+\frac{F_{5}^{\prime}(k, l, n) F_{5}^{\prime *}\left(k^{\prime},-l, n\right)}{\left(\omega_{m}^{2}+\lambda_{n}\right)\left(\omega_{m^{\prime}}^{2}+l^{2}\right)}\right) \\
& \left.+\int \frac{d l}{2 \pi \sqrt{q}} \frac{\tilde{F}_{5}^{\prime}(k, l, n) \tilde{F}_{5}^{\prime *}\left(k^{\prime},-l, n\right)}{\left(\omega_{m}^{2}\right)\left(\omega_{m^{\prime}}^{2}+l^{2}\right)}\right] \delta_{w+w^{\prime}}
\end{aligned}
$$

where, $w=m+m^{\prime}$. In (4.6), the first amplitude gets contributions from the fields $C_{m, n}$ with propagator (C.1) and $A_{x}^{3}\left(m^{\prime}, l\right)$ with propagator (C.5) in the loop with the threepoint vertex $F_{4}(k, l, n)$ given in (C.19). The corresponding Feynman diagram is given 


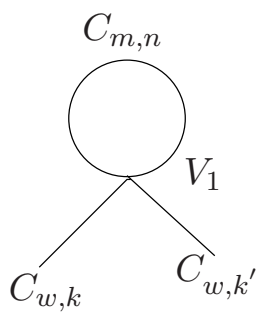

(a)

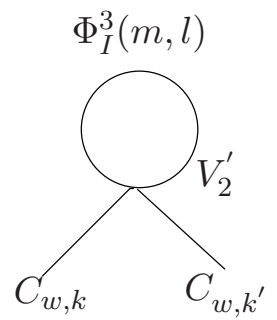

(d)

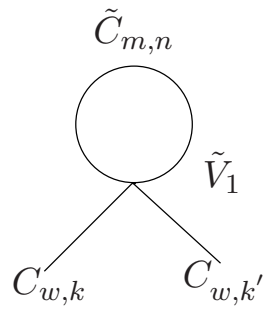

(b)

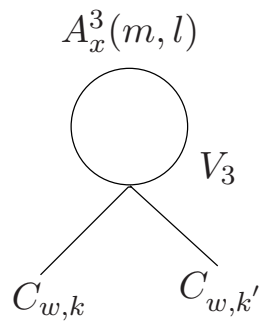

(e)

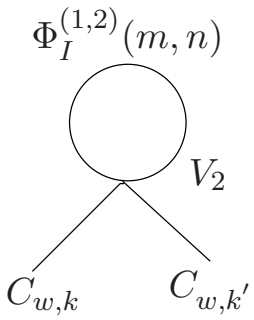

(c)

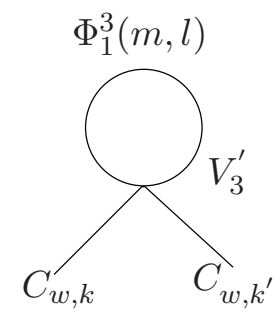

(f)

Figure 1. Feynman diagrams for the amplitudes with four-point vertices.

in figure 2(a). In the second amplitude, whose Feynman diagram is given by figure 2(b) comprises of the three-point vertex $\tilde{F}_{4}(k, l, n)$ given in (C.21) which gets contributions from the massless modes $\tilde{C}_{m, n}$ with propagator (C.2) and the massless gauge fields $A_{x}^{3}(m, l)$.

The third amplitude in (4.6) with the vertices $F_{5}(k, l, n)$, has contributions from the pairs of fields $\Phi_{I}^{(1,2)}(m, n)$ with propagator (C.3) and $\Phi_{I}^{3}(m, l)$ with propagator (C.4) and the Feynman diagram drawn in figure 2(c). The fourth amplitude consisting of the threepoint vertex $F_{5}^{\prime}(k, l, n)$ receives contributions in the loop from $C_{m, n}$. and $\Phi_{1}^{3}(m, l)$ with propagator (C.4), while the fifth one with vertex $\tilde{F}_{5}^{\prime}(k, l, n)$ comprises of the loop fields $\tilde{C}_{m, n}$ and $\Phi_{1}^{3}(m, l)$. The Feynman diagrams for the fourth and fifth terms in (4.6) are given in figure $2(\mathrm{~d})$ and figure $2(\mathrm{e})$ respectively.

We are interested in the two point function for the $w=w^{\prime}=k=k^{\prime}=0$ mode because this mode is the tachyon with the lowest mass $^{2}$ value. The finite temperature correction involves sum over the Matsubara frequency. Upon performing the Matsubara sum (except on the massless modes), the correction (4.5) for $w=w^{\prime}=0$ can be written as

$$
\begin{aligned}
\Sigma^{1}\left(0,0, k, k^{\prime}, \beta, q\right)=\frac{1}{2}[ & \sum_{n} \frac{F_{1}\left(k, k^{\prime}, n, n\right)}{\sqrt{(2 n-1)}}\left(\frac{1}{2}+\frac{1}{e^{\beta \sqrt{(2 n-1) q}}-1}\right) \\
& +N \sum_{m}\left(\sum_{n} \frac{\tilde{F}_{1}\left(k, k^{\prime}, n, n\right)}{\omega_{m}^{2}}+\int \frac{d l}{2 \pi \sqrt{q}} \frac{F_{3}\left(k, k^{\prime}, l-l\right)}{\omega_{m}^{2}}\right) \\
& +\sum_{n}\left(\frac{7 F_{2}\left(k, k^{\prime}, n, n\right)}{\sqrt{(2 n+1)}}\left(\frac{1}{2}+\frac{1}{e^{\beta \sqrt{(2 n+1) q}}-1}\right)\right) \\
& \left.+\left(\int \frac{d l}{2 \pi \sqrt{q}} \frac{(7+1 / 2) \delta_{k, k^{\prime}}}{(l / \sqrt{q})}\left(\frac{1}{2}+\frac{1}{e^{\beta l}-1}\right)\right)\right] .
\end{aligned}
$$




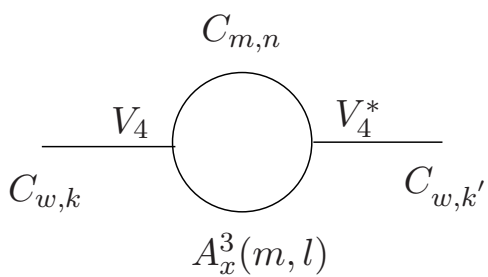

(a)

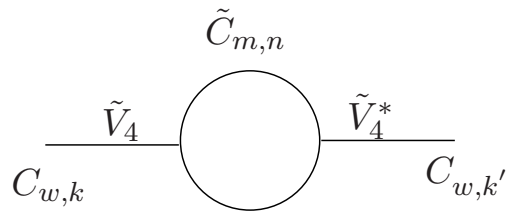

$A_{x}^{3}(m, l)$

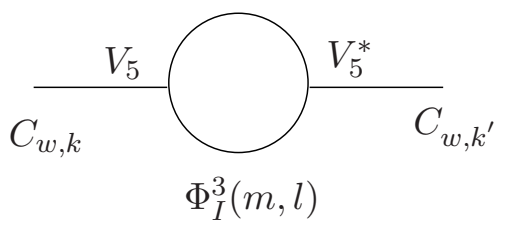

(b)

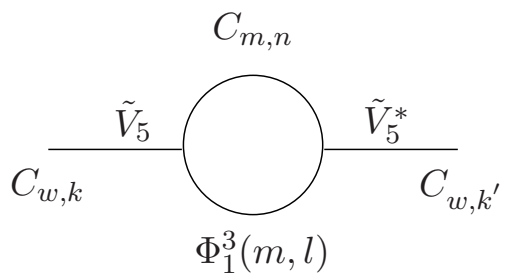

(d)

(c)

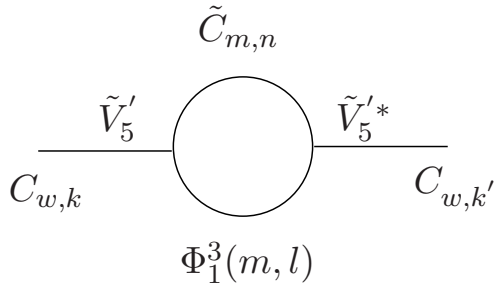

(e)

Figure 2. Feynman diagrams for the amplitudes with three-point vertices.

The correction given in (4.6), after the Matsubara sum (leaving out the massless modes) assumes the form

$$
\begin{aligned}
& \Sigma^{2}\left(0,0, k, k^{\prime}, \beta, q\right) \\
= & -\frac{1}{2} \sum_{n}\left[\int \frac{d l}{2 \pi \sqrt{q}} \frac{F_{4}(k, l, n) F_{4}^{*}\left(k^{\prime}, l, n\right)}{(2 n-1)}\left[\left(\sum_{m} \frac{\sqrt{q}}{\beta \omega_{m}^{2}}-\frac{1}{\sqrt{2 n-1}}\left(\frac{1}{2}+\frac{1}{e^{\sqrt{(2 n-1) q} \beta}-1}\right)\right)\right]\right. \\
& +q N \int \frac{d l}{2 \pi \sqrt{q}} \sum_{m} \frac{\tilde{F}_{4}(k, l, n) \tilde{F}_{4}^{*}\left(k^{\prime}, l, n\right)}{\omega_{m}^{4}} \\
& +\int \frac{d l}{2 \pi \sqrt{q}}\left[\frac { 7 F _ { 5 } ( k , l , n ) F _ { 5 } ^ { * } ( k ^ { \prime } , - l , n ) } { ( l / \sqrt { q } ) ^ { 2 } - ( 2 n + 1 ) } \left(\frac{1}{\sqrt{2 n+1}}\left(\frac{1}{2}+\frac{1}{e^{\sqrt{(2 n+1) q} \beta}-1}\right)\right.\right. \\
& \left.\left.-\frac{1}{(l / \sqrt{q})}\left(\frac{1}{2}+\frac{1}{e^{l \beta}-1}\right)\right)\right] \\
& +\int \frac{d l}{2 \pi \sqrt{q}}\left[\frac { F _ { 5 } ^ { \prime } ( k , l , n ) F _ { 5 } ^ { \prime * } ( k ^ { \prime } , - l , n ) } { ( l / \sqrt { q } ) ^ { 2 } - ( 2 n - 1 ) } \left(\frac{1}{\sqrt{2 n-1}}\left(\frac{1}{2}+\frac{1}{e^{\sqrt{(2 n-1) q} \beta}-1}\right)\right.\right. \\
& \left.\left.-\frac{1}{(l / \sqrt{q})}\left(\frac{1}{2}+\frac{1}{e^{l \beta}-1}\right)\right)\right] \\
& \left.+\int \frac{d l}{2 \pi \sqrt{q}} \frac{\tilde{F}_{5}^{\prime}(k, l, n) \tilde{F}_{5}^{\prime *}\left(k^{\prime},-l, n\right)}{(l / \sqrt{q})^{2}}\left(\sum_{m} \frac{\sqrt{q}}{\beta \omega_{m}^{2}}-\frac{1}{(l / \sqrt{q})}\left(\frac{1}{2}+\frac{1}{e^{l \beta}-1}\right)\right)\right] .
\end{aligned}
$$

In both (4.7) and (4.8), the Matsubara sums give rise to two different kinds of terms, the zero temperature quantum corrections and the temperature dependent part. While 
the zero-temperature parts are independent of $q$, the temperature dependent parts are functions of both $\beta$ and $q$. The temperature dependent terms are damped by exponential factors and are hence finite. The temperature independent parts however have problems of divergences. We shall discuss these problems in the section 5 .

\subsection{Fermions}

We will now compute the contribution to the tachyon two point amplitude due to fermionic fluctuations. The fermions in this contribution only appear in the internal loops. Consider first the free and the part of the action (A.13) in appendix A that couples to $\Phi_{1}^{3}$. The corresponding terms are,

$$
\begin{aligned}
\mathcal{L}_{1+1}^{2^{\prime}}= & \frac{1}{2}\left(\psi_{L}^{a T} \partial_{0} \psi_{L}^{a}+\psi_{R}^{a T} \partial_{0} \psi_{R}^{a}+\psi_{L}^{a T} \partial_{x} \psi_{L}^{a}-\psi_{R}^{a T} \partial_{x} \psi_{R}^{a}\right) \\
& +\Phi_{1}^{3}\left(\psi_{R}^{1 T} \alpha_{1}^{T} \psi_{L}^{2}-\psi_{R}^{2 T} \alpha_{1}^{T} \psi_{L}^{1}\right)
\end{aligned}
$$

We will now call the components of $\psi_{L}^{a}$ and those of $\psi_{R}^{a}$ as $L_{i}^{a}$ and $R_{i}^{a}$ respectively, where $a$ is the gauge index and $i=1, \cdots, 8$ is the fermion index. In this notation,

$$
\begin{aligned}
\mathcal{L}_{1+1}^{2^{\prime}}= & \frac{1}{2}\left(L_{i}^{a} \partial_{0} L_{i}^{a}+R_{i}^{a} \partial_{0} R_{i}^{a}+L_{i}^{a} \partial_{x} L_{i}^{a}-R_{i}^{a} \partial_{x} R_{i}^{a}\right) \\
& +\Phi_{1}^{3}\left(\psi_{R}^{1 T} \alpha_{1}^{T} \psi_{L}^{2}-\psi_{R}^{2 T} \alpha_{1}^{T} \psi_{L}^{1}\right) .
\end{aligned}
$$

With the background value of $\Phi_{1}^{3}=q x$ and putting in the value of $\alpha_{1}$ from (A.9), we proceed to diagonalize the action. This amounts to solving for the eigenfunctions of $L_{i}^{a}$ and $R_{i}^{a}$. We have the following sets of equations from (4.10).

$$
\begin{aligned}
\left(\partial_{0}+\partial_{x}\right) L_{1}^{1}+q x R_{8}^{2} & =0 \\
\left(-\partial_{0}+\partial_{x}\right) R_{8}^{2}+q x L_{1}^{1} & =0 \\
\left(\partial_{0}+\partial_{x}\right) L_{1}^{2}-q x R_{8}^{1} & =0 \\
\left(-\partial_{0}+\partial_{x}\right) R_{8}^{1}-q x L_{1}^{2} & =0 .
\end{aligned}
$$

There are sixteen such sets of decoupled equations. The eight sets

$$
\left(L_{1}^{1}, R_{8}^{2}\right),\left(L_{4}^{1}, R_{5}^{2}\right),\left(L_{6}^{1}, R_{3}^{2}\right),\left(L_{7}^{1}, R_{2}^{2}\right),\left(L_{2}^{2}, R_{7}^{1}\right),\left(L_{3}^{2}, R_{6}^{1}\right),\left(L_{5}^{2}, R_{4}^{1}\right),\left(L_{8}^{2}, R_{1}^{1}\right)
$$

satisfy identical coupled equations like (4.11), (4.12). The remaining eight sets

$$
\left(L_{1}^{2}, R_{8}^{1}\right),\left(L_{4}^{2}, R_{5}^{1}\right),\left(L_{6}^{2}, R_{3}^{1}\right),\left(L_{7}^{2}, R_{2}^{1}\right),\left(L_{8}^{1}, R_{1}^{2}\right),\left(L_{5}^{1}, R_{4}^{2}\right),\left(L_{3}^{1}, R_{6}^{2}\right),\left(L_{2}^{1}, R_{7}^{2}\right)
$$

satisfy coupled equations like (4.13), (4.14). For future convenience we denote the left and right fermions of (4.15)as $L(x, t)$ and $R(x, t)$ and those of $(4.16)$ as $\hat{L}(x, t)$ and $\hat{R}(x, t)$. Now we solve the differential equations. The set of coupled differential equations (4.11), (4.12) satisfied by the set of fermionic fields in (4.15) can be promoted to the status of second order differential equations as

$$
\begin{aligned}
& \left(-\partial_{0}^{2}+\partial_{x}^{2}\right) L(x, t)+q R(x, t)-q^{2} x^{2} L(x, t)=0 \\
& \left(-\partial_{0}^{2}+\partial_{x}^{2}\right) R(x, t)+q L(x, t)-q^{2} x^{2} R(x, t)=0 .
\end{aligned}
$$


It is important to note that the set of fermions in (4.16) also satisfy the same set of equations as (4.17), (4.18) with $L(x, t)$ and $R(x, t)$ being replaced by $\hat{L}(x, t)$ and $-\hat{R}(x, t)$ respectively. Let us discuss the solutions to the equations (4.17), (4.18). Adding (4.17) and (4.18) and subtracting (4.18) from (4.17) we get the following set of equations

$$
\begin{aligned}
& \left(-\partial_{0}^{2}+\partial_{x}^{2}\right) F(x, t)+q F(x, t)-q^{2} x^{2} F(x, t)=0 \\
& \left(-\partial_{0}^{2}+\partial_{x}^{2}\right) G(x, t)-q G(x, t)-q^{2} x^{2} G(x, t)=0
\end{aligned}
$$

where $F(x, t)=L(x, t)+R(x, t)$ and $G(x, t)=L(x, t)-R(x, t)$. In this context let us point out that one can construct similar combinational functions with the fields in (4.16) viz. $\hat{F}(x, t)=\hat{L}(x, t)+\hat{R}(x, t)$ and $\hat{G}(x, t)=\hat{L}(x, t)-\hat{R}(x, t)$, where $\hat{F}=G$ and $\hat{G}=F$. As in the case of bosonic fields, the fermionic differential equations can also be analyzed in the asymptotic limit and the fermionic eigenfunctions can be written as

$$
\begin{aligned}
& L_{i}^{a}(t, x)=e^{-\frac{q x^{2}}{2}} \tilde{L}_{i}^{a}(x, t) \\
& R_{i}^{a}(t, x)=e^{-\frac{q x^{2}}{2}} \tilde{R}_{i}^{a}(x, t) .
\end{aligned}
$$

Note that although there are two different sets of coupled differential equations; one being (4.11), (4.12) satisfied by (4.15) and the other (4.13), (4.14) satisfied by (4.16), both sets of equations when recombined give rise to the same differential equations as (4.17) for the left moving fermions and (4.18) for the right moving fermions. The eigenfunctions from (4.17) and (4.18) that also satisfies the first order equations (4.11) are given by

$$
\psi_{n}(x)=\left(\begin{array}{c}
L_{n}(x) \\
R_{n}(x)
\end{array}\right) .
$$

The corresponding eigenvalue is $=-i \sqrt{\lambda^{\prime}}=-i \sqrt{2 n q}$. Similarly for the set of fermions given in (4.16) and obeying the equations of motion (4.13), the eigenfunctions can be obtained by repeating the above procedure and we get

$$
\hat{\psi}_{n}(x)=\left(\begin{array}{c}
\hat{L}_{n}(x) \\
\hat{R}_{n}(x)
\end{array}\right)=\left(\begin{array}{c}
L_{n}(x) \\
-R_{n}(x)
\end{array}\right)
$$

where,

$$
\begin{aligned}
& L_{n}(x)=\hat{L}_{n}(x)=\mathcal{N}_{F} e^{-\frac{q x^{2}}{2}}\left(-\frac{i}{\sqrt{2 n}} H_{n}(\sqrt{q} x)+H_{n-1}(\sqrt{q} x)\right) \\
& R_{n}(x)=-\hat{R}_{n}(x)=\mathcal{N}_{F} e^{-\frac{q x^{2}}{2}}\left(-\frac{i}{\sqrt{2 n}} H_{n}(\sqrt{q} x)-H_{n-1}(\sqrt{q} x)\right) .
\end{aligned}
$$

$H_{n}(\sqrt{q} x)$ are the Hermite Polynomials. The normalization $\mathcal{N}_{F}=\sqrt{\sqrt{\pi} 2^{n+1}(n-1) !}$. We now list some important relations satisfied by the eigenfunctions

$$
\sqrt{q} \int d x \psi_{n}^{\dagger}(x) \psi_{n^{\prime}}(x)=\sqrt{q} \int d x\left(L_{n}^{*}(x) L_{n^{\prime}}(x)+R_{n}^{*}(x) R_{n^{\prime}}(x)\right)=\delta_{n, n^{\prime}} .
$$




$$
\begin{aligned}
& \sqrt{q} \int d x L_{n}^{*}(x) L_{n^{\prime}}(x)=\sqrt{q} \int d x R_{n}^{*}(x) R_{n^{\prime}}(x)=\frac{1}{2} \delta_{n, n^{\prime}} \\
& \sqrt{q} \int d x \psi_{n}^{T}(x) \psi_{n^{\prime}}(x)=\sqrt{q} \int d x\left(L_{n}(x) L_{n^{\prime}}(x)+R_{n}(x) R_{n^{\prime}}(x)\right)=0 \\
& \sqrt{q} \int d x \psi_{n}^{\dagger}(x) \psi_{n^{\prime}}^{*}(x)=\sqrt{q} \int d x\left(L_{n}^{*}(x) L_{n^{\prime}}^{*}(x)+R_{n}^{*}(x) R_{n^{\prime}}^{*}(x)\right)=0 .
\end{aligned}
$$

With the eigenfunctions as defined above, we can now write down the mode expansions for the sixteen pairs defined in (4.15) and (4.16). For example we write,

$$
\left(\begin{array}{l}
L_{1}^{1}(x, \tau) \\
R_{8}^{2}(x, \tau)
\end{array}\right)=N^{3 / 4} \sum_{n, m=\infty}^{\infty}\left(\theta_{1}(m, n) e^{i \omega_{m} \tau}\left(\begin{array}{c}
L_{n}(x) \\
R_{n}(x)
\end{array}\right)+\theta_{1}^{*}(m, n) e^{-i \omega_{m} \tau}\left(\begin{array}{c}
L_{n}^{*}(x) \\
R_{n}^{*}(x)
\end{array}\right)\right)
$$

where we have used $\tau=i t$ and $N=\sqrt{q} / \beta$. For each doublet in (4.15) and (4.16) we have a

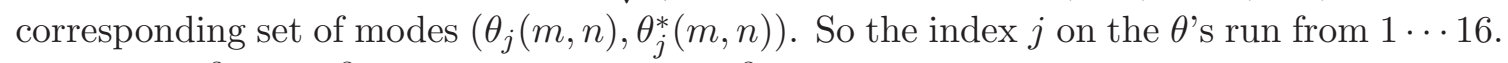

Since $L_{i}^{3}$ and $R_{i}^{3}$ do not couple to the $\Phi_{1}^{3}=q x$ background, they just satisfy the plane wave equations,

$$
\left(\partial_{0}+\partial_{x}\right) L_{i}^{3}=0 ; \quad\left(-\partial_{0}+\partial_{x}\right) R_{i}^{3}=0 .
$$

Hence their mode expansions are

$$
\begin{aligned}
& L_{i}^{3}(x, \tau)=N^{3 / 4} \sum_{m} \frac{1}{\sqrt{q}} \int \frac{d k}{(2 \pi)} L_{i}^{3}(m, k) e^{i\left(k x+\omega_{m} \tau\right)} \\
& R_{i}^{3}(x, \tau)=N^{3 / 4} \sum_{m} \frac{1}{\sqrt{q}} \int \frac{d k}{(2 \pi)} R_{i}^{3}(m, k) e^{i\left(k x+\omega_{m} \tau\right)} \quad \text { for all } i=1, \cdots, 8 .
\end{aligned}
$$

where $L_{i}^{3 *}(m, k)=L_{i}^{3}(m, k)$.

Using the orthogonality relations and the mode expansions the quadratic part of the fermionic action can thus be written as,

$$
\begin{aligned}
S_{f}=\frac{N^{1 / 2}}{g^{2}}\left[\sum_{m, n, j=1}^{j=16} \theta_{j}(m, n)\left(i \omega_{m}-\sqrt{\lambda_{n}^{\prime}}\right) \theta_{j}^{*}(m, n)\right. \\
\quad+\frac{1}{2 \sqrt{q}} \int \frac{d k}{2 \pi} \sum_{m, i=1}^{i=8} L_{i}^{3}(m, k)\left(i \omega_{m}+k\right) L_{i}^{3 *}(m, k) \\
\left.\quad+\frac{1}{2 \sqrt{q}} \int \frac{d k}{2 \pi} \sum_{m, i=1}^{i=8} R_{i}^{3}(m, k)\left(i \omega_{m}-k\right) R_{i}^{3 *}(m, k)\right] .
\end{aligned}
$$

With these we can write down the fermionic propagators as listed in the appendix. We now turn to the interaction terms. These are the terms in the fermionic action (4.10) that couple to the background fields $\Phi_{1}^{1}$ and $A_{x}^{2}$ that we simply call $\phi_{B}$ and $A_{B}$ respectively as before.

$$
\mathcal{L}_{f}=\phi_{B} \psi_{R}^{2 T} \alpha_{1}^{T} \psi_{L}^{3}-\phi_{B} \psi_{R}^{3 T} \alpha_{1}^{T} \psi_{L}^{2}+A_{B} \psi_{L}^{1 T} \psi_{L}^{3}-A_{B} \psi_{R}^{1 T} \psi_{R}^{3}
$$




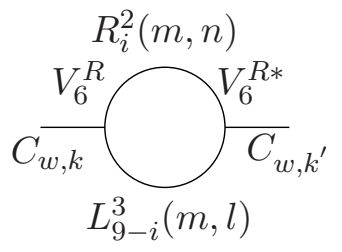

(a)

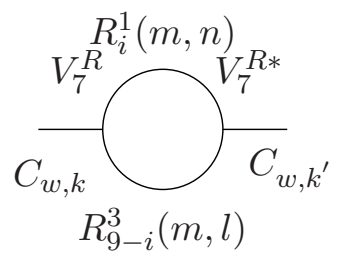

(c)

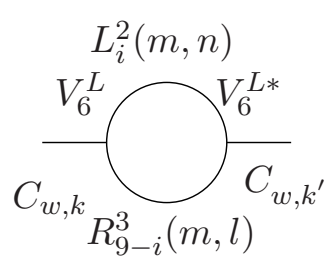

(b)

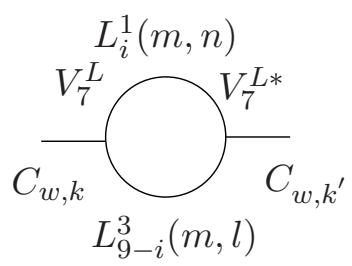

(d)

Figure 3. Feynman diagrams for the amplitudes with three-point fermionic vertices $V^{R / L}$ and their complex conjugates $V^{* R / L}$.

The corresponding vertices have been worked out in appendix. We thus have the following contribution to the tachyon two-point amplitude.

$$
\begin{aligned}
\Sigma^{3}\left(w, w^{\prime}, k, k^{\prime}, \beta, q\right)= & -(8 N) \sum_{n, m, m^{\prime}} \int \frac{d l}{2 \pi \sqrt{q}} \frac{1}{\left(i \omega_{m}-\sqrt{\lambda_{n}^{\prime}}\right)} \\
\times & {\left[\frac{F_{6}^{R}(k, n, l) F_{6}^{R *}\left(k^{\prime}, n, l\right)}{\left(i \omega_{m^{\prime}}+l\right)}+\frac{F_{6}^{L}(k, n, l) F_{6}^{L *}\left(k^{\prime}, n, l\right)}{\left(i \omega_{m^{\prime}}-l\right)}\right.} \\
& +\frac{F_{7}^{L}(k, n, l) F_{7}^{L *}\left(k^{\prime}, n, l\right)}{\left(i \omega_{m^{\prime}}+l\right)}+\frac{F_{7}^{R}(k, n, l) F_{7}^{R *}\left(k^{\prime}, n, l\right)}{\left(i \omega_{m^{\prime}}-l\right)} \\
& +\frac{F_{6}^{R}(k, n, l) F_{7}^{L *}\left(k^{\prime}, n, l\right)+F_{6}^{R *}(k, n, l) F_{7}^{L}\left(k^{\prime}, n, l\right)}{\left(i \omega_{m^{\prime}}+l\right)} \\
& \left.+\frac{F_{6}^{L}(k, n, l) F_{7}^{R *}\left(k^{\prime}, n, l\right)+F_{6}^{L *}(k, n, l) F_{7}^{R}\left(k^{\prime}, n, l\right)}{\left(i \omega_{m^{\prime}}-l\right)}\right] \delta_{w+w^{\prime}}
\end{aligned}
$$

where $w=m+m^{\prime}$. In (4.35) the Matsubara frequency $\omega_{m}=m \pi / \beta$ and $m$ is an odd integer due to anti-periodic boundary conditions along the time-cycle for the fermions and $\omega_{-m}=-\omega_{m}$. The various diagrams contributing to the amplitude is shown in figures 3 and 4 . Figure 4 shows the contractions between $R_{i}^{2}-L_{9-i}^{1}$ and $L_{i}^{2}-R_{9-i}^{1}$ as they are expanded with the same $\theta$ 's. The first term within 3 rd bracket in (4.35) is represented by the Feynman diagram in figure 3(a), while the second term by figure 3(b). The third and fourth terms are represented by figure 3(c) and figure 3(d) respectively.

The fifth and sixth terms in (4.35) results from the "cross"-contraction between the right-moving and left-moving fermions and are depicted in the Feynman diagrams in figures 4(a), 4(b) respectively. The functions $F_{6}^{R / L}$ and $F_{7}^{R / L}$ are all given in eqns (C.32), 


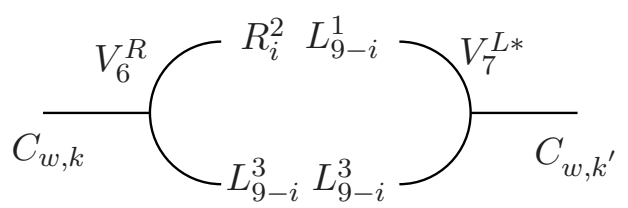

(a)

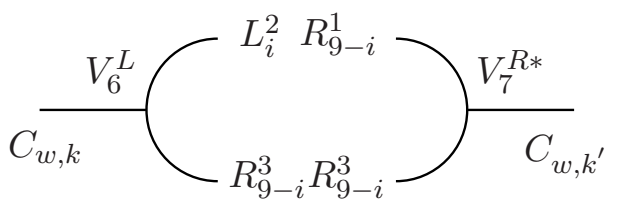

(b)

Figure 4. Feynman diagrams showing the cross terms in the amplitude with fermions in the loop.

(C.31) and (C.34). The massive fermions namely $L_{i}^{(1,2)}$ and $R_{i}^{(1,2)}$ have their propagators given by (C.28). The propagators for the massless fermions which are the 3rd gauge component of the fermionic fields namely $L_{i}^{3}$ and $R_{i}^{3}$ are given in (C.29).

After performing the Matsubara sum in (4.35), the fermionic contribution to the oneloop mass-squared corrections for the tree-level tachyon can be written as

$$
\begin{aligned}
& \Sigma^{3}\left(0,0, k, k^{\prime}, \beta, q\right)=(8 N) \sum_{n}\left[\int \frac{d l}{2 \pi \sqrt{q}}\left(\frac{-\beta \tanh \left(\frac{\beta l}{2}\right)-\beta \tanh \left(\frac{1}{2} \beta \sqrt{2 n q}\right)}{2(l+\sqrt{2 n q})}\right)\right. \\
& {\left[F_{6}^{R}(k, n, l) F_{6}^{R *}\left(k^{\prime}, n, l\right)+F_{7}^{L}(k, n, l) F_{7}^{L *}\left(k^{\prime}, n, l\right)\right.} \\
& \left.+F_{6}^{R}(k, n, l) F_{7}^{L *}\left(k^{\prime}, n, l\right)+F_{6}^{R *}(k, n, l) F_{7}^{L}\left(k^{\prime}, n, l\right)\right] \\
& +\int \frac{d l}{2 \pi \sqrt{q}}\left(\frac{-\beta \tanh \left(\frac{\beta l}{2}\right)+\beta \tanh \left(\frac{1}{2} \beta \sqrt{2 n q}\right)}{2(l-\sqrt{2 n q})}\right) \\
& +\left[F_{6}^{L}(k, n, l) F_{6}^{L *}\left(k^{\prime}, n, l\right)+F_{7}^{R}(k, n, l) F_{7}^{R *}\left(k^{\prime}, n, l\right)\right. \\
& \left.\left.+F_{6}^{L}(k, n, l) F_{7}^{R *}\left(k^{\prime}, n, l\right)+F_{6}^{L *}(k, n, l) F_{7}^{R}\left(k^{\prime}, n, l\right)\right]\right] .
\end{aligned}
$$

As found in the case of bosonic amplitudes the fermionic counterpart given by (4.36) also can be regrouped into two different parts the zero-temperature quantum corrections and the finite temperature pieces. The amplitudes containing fermions in the loop are infrared finite because of anti-periodic boundary conditions imposed on the fermions whereby they pick up temperature dependent mass at tree-level. However the fermionic amplitudes (4.36) are ultraviolet divergent. The divergence come from the temperature independent pieces in (4.36). We shall discuss this problem in details in the following section.

\section{The ultraviolet and infrared problems}

Each integral as well as the terms bearing the contribution from the massless modes $C_{w, k}$ in (4.7) and (4.8) are infrared divergent for $\left(\omega_{m}=0, l=0\right)$. Moreover the sums over the momentum $n$ do not converge and integral over the momentum $l$ are log divergent. These give rise to ultraviolet divergence in each term in the two-point functions in (4.7), (4.8) and (4.36). We deal with the ultraviolet problem first. 


\subsection{Ultraviolet finiteness of tachyonic amplitudes}

As mentioned above, every term in the two-point functions (4.5), (4.6) and (4.35) is ultraviolet divergent. In the present scenario supersymmetry is completely broken by choice of background, namely, $\left\langle\Phi_{1}^{3}\right\rangle=q x$, however the equality in the number of bosonic and fermionic degrees of freedom still holds good in the intersecting brane configuration. In the ultraviolet limit the degeneracy in the masses of the bosons and fermions is restored and the ultraviolet divergences from the bosonic terms cancel with that from the fermionic terms. We now proceed to show this cancellation.

After performing the Matsubara sum (see appendix F), the temperature independent part of the bosonic propagators can be written as

$$
\begin{aligned}
\frac{1}{\omega_{m}^{2}+\lambda_{n}} & \rightarrow \frac{1}{2 \sqrt{\lambda_{n}}} \\
\frac{1}{\omega_{m}^{2}+\gamma_{n}} & \rightarrow \frac{1}{2 \sqrt{\gamma_{n}}} \\
\frac{1}{\omega_{m}^{2}+l^{2}} & \rightarrow \frac{1}{2 l} \\
\frac{1}{\left(\omega_{m}^{2}+\lambda_{n}\right)\left(\omega_{m}^{2}+\gamma_{n^{\prime}}\right)} & \rightarrow \frac{1}{\gamma_{n^{\prime}} \sqrt{\lambda_{n}}\left(\gamma_{n^{\prime}}+\sqrt{\lambda_{n}}\right)} .
\end{aligned}
$$

Let us now look at the various four-point and three-point vertices. We compute the UV limit of the amplitudes for external momentum $k=0=k^{\prime}$. This computation gives rise to Gamma functions summed over their arguments. For UV behaviour we take asymptotic expansion of the Gamma functions. We first compute the four-pont vertices in the bosonic corrections (4.5) in the limit $n \rightarrow \infty$. For one-loop calculation $n=n^{\prime}$. We use the following properties of $\Gamma(*)$ functions:

$$
\begin{aligned}
\lim _{n \rightarrow \infty} \Gamma(n+1) & \sim\left(\frac{n}{e}\right)^{n} \sqrt{2 \pi n} \\
\Gamma\left(n+\frac{1}{2}\right) & =\frac{2 n !}{4^{n} n !} \sqrt{\pi} .
\end{aligned}
$$

Also the asymptotic expansion of the Hermite Polynomials for $n \rightarrow \infty$ gives

$$
e^{-\frac{x^{2}}{2}} H_{n}(x) \sim \frac{2^{n}}{\sqrt{\pi}} \Gamma\left(\frac{n+1}{2}\right) \cos \left(\sqrt{2 n} x-n \frac{\pi}{2}\right) .
$$

With this asymptotic expansions at our disposal, we can now compute the four-point bosonic vertices. The four-point vertex $F_{1}(0,0, n, n)$ can be written as (using the results of appendix B, C)

$$
F_{1}(0,0, n, n)=\frac{\mathcal{N}^{2}(n)}{2 \sqrt{\pi}} \int_{\infty}^{\infty} d x e^{-2 \sqrt{q} x^{2}}\left(6 H_{n}(\sqrt{q} x) H_{n}(\sqrt{q} x)-8 n^{2} H_{n-2}(\sqrt{q} x) H_{n-2}(\sqrt{q} x)\right)
$$


Using the asymptotic expansion of the Hermite polynomials given in (5.7), we get

$$
\begin{aligned}
F_{1}(0,0, n, n) & =\frac{\mathcal{N}^{2}(n) \sqrt{q}}{2 \sqrt{\pi}} \int_{-\infty}^{\infty} d x e^{-2 \sqrt{q} x^{2}}\left(6\left(H_{n}(\sqrt{q} x)\right)^{2}-8 n^{2}\left(H_{n-2}(\sqrt{q} x)\right)^{2}\right) \\
& =\frac{2^{2 n+1} \mathcal{N}^{2}(n) \Gamma^{2}\left(\frac{n+1}{2}\right)}{2 \pi} \sqrt{q} \int_{-\infty}^{\infty} d x e^{-\sqrt{q} x^{2}} \cos ^{2}\left(\sqrt{2 n q} x-n \frac{\pi}{2}\right) \\
& =\left(1+\frac{(-1)^{n}}{e^{2 n}}\right) \frac{2^{2 n}\left(\Gamma\left(\frac{n+1}{2}\right)\right)^{2}}{\pi^{2} 2^{n}(4 n-2) n(n-2) !} .
\end{aligned}
$$

In the ultraviolet limit the vertex function $F_{1}(0,0, n, n)$ reduces to

$$
\left.F_{1}(0,0, n, n)\right|_{n \rightarrow \infty}=\left.\left(\left(1+\frac{(-1)^{n}}{e^{2 n}}\right) \frac{2^{2 n}\left(\Gamma\left(\frac{n+1}{2}\right)\right)^{2}}{\pi^{2} 2^{n}(4 n-2) n(n-2) !}\right)\right|_{n \rightarrow \infty}=\frac{1}{2 \pi \sqrt{2 n}} .
$$

The vertex $\tilde{F}_{1}(0,0, n, n)$ has a similar form and in the ultraviolet limit also reduces to

$$
\left.\tilde{F}_{1}(0,0, n, n)\right|_{n \rightarrow \infty}=\left.\left(\left(1+\frac{(-1)^{n}}{e^{2 n}}\right) \frac{2^{2 n}\left(\Gamma\left(\frac{n+1}{2}\right)\right)^{2}}{\pi^{2} 2^{n}(4 n-2)(n-1) !}\right)\right|_{n \rightarrow \infty}=\frac{1}{2 \pi \sqrt{2 n}} .
$$

Putting the external momenta $k=k^{\prime}=0$, the four-point vertex function $F_{2}(0,0, n, n)$ in the limit $n \rightarrow \infty$ can be written as

$$
\begin{aligned}
F_{2}(0,0, n, n) & =\frac{1}{2^{n+1} \sqrt{\pi} n !} \sqrt{q} \int_{-\infty}^{\infty} d x e^{-2 \sqrt{q} x^{2}}\left(H_{n}(\sqrt{q} x)\right)^{2} \\
& =\left(1+\frac{(-1)^{n}}{e^{2 n}}\right) \frac{2^{2 n}\left(\Gamma\left(\frac{n+1}{2}\right)\right)^{2}}{\pi^{2} 2^{n+1} n !} .
\end{aligned}
$$

In the UV limit the vertex function (5.12) reduces to

$$
\left.F_{2}(0,0, n, n)\right|_{n \rightarrow \infty}=\left.\left(1+\frac{(-1)^{n}}{e^{2 n}}\right) \frac{2^{2 n}\left(\Gamma\left(\frac{n+1}{2}\right)\right)^{2}}{\pi^{2} 2^{n+1} n !}\right|_{n \rightarrow \infty}=\frac{1}{\pi \sqrt{2 n}} .
$$

The remaining four-point bosonic vertex functions namely $F_{2}^{\prime}(0,0, l,-l), F_{3}(0,0)$ and $F_{3}^{\prime}(0,0, l,-l)$ are independent of $n$. Therefore for a fixed value of the external momenta, namely $k=k^{\prime}=0$ they can be exactly computed and found to be,

$$
\begin{aligned}
F_{2}^{\prime}(0,0, l,-l) & =1 \\
F_{3}^{\prime}(0,0, l,-l) & =\frac{1}{2} \\
F_{3}(0,0, l,-l) & =\frac{1}{2} .
\end{aligned}
$$

The three-point bosonic vertices contain both the continuous momentum $l$ coming from the massless fields as well as the discrete momentum $n$ coming from the fields coupled to the background $\left\langle\phi_{1}^{3}\right\rangle=q x$. The UV-limit must be taken unambiguously for each term in the amplitude $\Sigma^{2}(0,0,0,0, \beta, q)$. Let us first try to compute the amplitude for the three-point 
vertices $F_{4}(0, l, n)$ and $\tilde{F}_{4}(0, l, n)$ with external momenta $k=0$. The three-point vertex $F_{4}(0, l, n)$ can be written as

$$
F_{4}(0, l, n)=\frac{\mathcal{N}(n)}{\sqrt{2 \sqrt{\pi}}} \sqrt{q} \int d x e^{-q x^{2}} e^{i l x} 4 n H_{n}(\sqrt{q} x)
$$

where $\mathcal{N}(n)$ are the normalization factors for the eigenvectors $\zeta_{n}(x)$ and the factor $e^{i l x}$ can be attributed to the presence of the massless field $A_{x}^{3}(m, l)$ in the loop. Hence the integral in (5.18) is simply the Fourier transform of the Hermite polynomials weighted by Gaussian factor.

The Fourier transform of a single Hermite Polynomial is given by

$$
\int_{-\infty}^{\infty} d x\left(e^{i l x} e^{-x^{2}} H_{n}(x)\right)=(-1)^{n} i^{n} \sqrt{\pi} e^{-\frac{l^{2}}{4}} l^{n} .
$$

In the following analysis we will set $q=1$ and will restore factors of $q$ only in the final expressions. Using (5.19) in (5.18) the three-point vertex $F_{4}(0, l, n)$ can be written as

$$
F_{4}(0, l, n)=\sqrt{\pi}(-1)^{n-1} i^{n-1}\left(\frac{4 n e^{-\frac{l^{2}}{4}} l^{n-1}}{\sqrt{2^{n+1} \pi\left(4 n^{2}-2 n\right)(n-2) !}}\right) .
$$

We decompose the corresponding propagator into partial fraction (for reference see the first term in (4.6) and the Feynman diagram in figure 2(a)).

$$
\frac{1}{\omega_{m}^{2}\left(\omega_{m}^{2}+\lambda_{n}\right)}=\frac{1}{\lambda_{n}}\left(\frac{1}{\omega_{m}^{2}}-\frac{1}{\left(\omega_{m}^{2}+\lambda_{n}\right)}\right) .
$$

The amplitude comprising of the vertex $F_{4}(0, l, n)$ given in (4.6) is given by

$$
\sum_{m, n} \int \frac{d l}{2 \pi}\left(\frac{16 n^{2} e^{-\frac{l^{2}}{2}} l^{2 n-2}}{2^{n+1}\left(4 n^{2}-2 n\right)(n-2) !} \frac{1}{\lambda_{n}} \frac{1}{\omega_{m}^{2}}-\frac{16 n^{2} e^{-\frac{l^{2}}{2}} l^{2 n-2}}{2^{n+1}\left(4 n^{2}-2 n\right)(n-2) !} \frac{1}{\lambda_{n}\left(\omega_{m}^{2}+\lambda_{n}\right)}\right)
$$

In the first term in (5.22) we perform the sum over $n$ and take the UV limit $l \rightarrow$ $\infty$. In the second term we first compute the integration over $l$ and then expand the resulting expression asymptotically about $n=\infty$. The leading order contribution to the UV divergence obtained from this amplitude is thus

$$
\sum_{m} \int \frac{d l}{2 \pi \sqrt{q}} \frac{1}{2 \omega_{m}^{2}}-\sum_{m, n} \frac{1}{2 \pi \sqrt{2 n}} \frac{1}{\omega_{m}^{2}+\lambda_{n}} .
$$

The three-point vertex $\tilde{F}_{4}(0, l, n)$ vanishes for all $n$. Hence the corresponding amplitude vanishes. The two-point functions corresponding to the three-point vertices $F_{5}(0, l, n)$, $F_{5}^{\prime}(0, l, n)$ and $\tilde{F}_{5}^{\prime}(0, l, n)$ (obtained from (C.22), (C.24) and (C.26)) contain propagators with momentum $l$ as well as those containing the momentum $n$. The amplitude bearing the three-point vertex $F_{5}(0, l, n)$ contains contributions from the fields $\Phi_{I}^{1,2}(m, n)$ and the massless fields $\Phi_{I}^{3}(m, l)$ in the loop. Therefore the propagators contain both the mass-squared 
eigenvalues $\gamma_{n}=(2 n+1) q$ of the basis functions of the fields $\Phi_{I}^{1,2}$ and the momentum $l$ of the massless field $\Phi_{I}^{3}$. Similarly $F_{5}^{\prime}(0, l, n)$ has contributions from the fields $C_{m, n}$ and the massless fields $\Phi_{1}^{3}(m, l)$. Therefore the propagators in the corresponding two-point function has both the mass-squared eigenvalues $\lambda_{n}=(2 n-1) q$ and the momentum $l$. In both these amplitudes we first perform the integration over $l$ and then asymptotically expand the resulting expressions about $n=\infty$. As for the two-point function for the three-point vertex $\tilde{F}_{5}^{\prime}(0, l, n)$ the fields participating in the loop are the massless modes $\tilde{C}_{m, n}$ as well as $\Phi_{1}^{3}(m, l)$. In this case we first decompose the mixed propagator into two parts. We then sum over $n$ and then take the limit $l \rightarrow \infty$. Throughout the computations the external momentum is kept fixed at $k=0$. All the three-point vertices $F_{5}(0, l, n), F_{5}^{\prime}(0, l, n)$ and $\tilde{F}_{5}^{\prime}(0, l, n)$ has the factor $e^{i l x}$ due to the presence of massless fields in the loop. As in the cases of $F_{4}(0, l, n)$, the integrals over the world-volume coordinate $x$ in these vertex functions amount to evaluating the the Fourier transform of the various Hermite polynomials constituting the vertex functions.

Using the result from (5.19) in computing the vertex functions (C.22), (C.24) and (C.26) for $k=0$, we arrive at the following expressions

$$
\begin{aligned}
& F_{5}(0, l, n)=-(-1)^{n+1} i^{(n+1)} e^{-\frac{l^{2}}{4}} \frac{\left(l^{(n+1)}+2 n l^{(n-1)}\right)}{\sqrt{2^{n+1} \pi n !}} \\
& F_{5}^{\prime}(0, l, n)=(-1)^{n+1} i^{(n+1)} e^{-\frac{l^{2}}{4}} \frac{3 l^{(n+1)}+4 n(n-2) l^{(n-3)}}{\sqrt{2^{n+2} \pi\left(4 n^{2}-2 n\right)(n-2) !}} \\
& \tilde{F}_{5}^{\prime}(0, l, n)=(-1)^{n+1} i^{(n+1)} e^{-\frac{l^{2}}{4}} \frac{3 l^{(n+1)}-4 n(n-2) l^{(n-3)}}{\sqrt{2^{n+2} \pi(4 n-2)(n-1) !}}
\end{aligned}
$$

The amplitude for the three-point vertex $F_{5}(0, l, n)$ can be written as

$$
\sum_{m, n} \int \frac{d l}{2 \pi} \frac{F_{5}(0, l, n) F_{5}^{*}(0, l, n)}{\left(\omega_{m}^{2}+\gamma_{n}\right)\left(\omega_{m}^{2}+l^{2}\right)}=\sum_{m, n} \int \frac{d l}{2 \pi} \frac{e^{-\frac{l^{2}}{2}}}{\left(\omega_{m}^{2}+\gamma_{n}\right)\left(\omega_{m}^{2}+l^{2}\right)} \frac{\left(l^{(n+1)}+2 n l^{(n-1)}\right)^{2}}{2^{n+1} \pi n !}
$$

where the second line in (5.27) is obtained by plugging in (5.24) in the first line. After performing the integral over $l$, we asymptotically expand the result about $n=\infty$ This results into

$$
\begin{aligned}
& \sum_{m, n} \int \frac{d l}{2 \pi} \frac{F_{5}(0, l, n) F_{5}^{*}(0, l, n)}{\left(\omega_{m}^{2}+\gamma_{n}\right)\left(\omega_{m}^{2}+l^{2}\right)} \\
= & \frac{1}{2} \sum_{m, n} \frac{1}{\left(\omega_{m}^{2}+\gamma_{n}\right)}\left[\left(\frac{2 \sqrt{2} \sqrt{\frac{1}{n}}}{\pi}-\frac{\left(\left(-7+4 \omega_{m}^{2}\right)\right)\left(\frac{1}{n}\right)^{3 / 2}}{2(\sqrt{2} \pi)}+\mathcal{O}\left(\frac{1}{n}\right)^{2}\right)\right. \\
& \left.+2^{-n}\left(\frac{e}{n}\right)^{n}\left(\omega_{m}^{2}\right)^{n} \sec (n \pi)\left(-\frac{e^{\frac{\omega_{m}^{2}}{2}} \sqrt{\frac{2}{\pi}} n^{3 / 2}}{\omega_{m}^{3}}+\frac{e^{\frac{\omega_{m}^{2}}{2}}\left(24 \omega_{m}^{2}+2\right) \sqrt{n}}{12 \sqrt{2 \pi} \omega_{m}^{3}}+\mathcal{O}\left(\frac{1}{n}\right)^{0}\right)\right] .
\end{aligned}
$$

In the above expression the terms with odd power of $\omega_{m}$ vanishes under summation over $m$ over $\{-\infty, \infty\}$. The leading order term in the amplitude contributing to UV 
divergence is

$$
\sum_{m, n} \frac{4}{2 \pi \sqrt{2 n}} \frac{1}{\left(\omega_{m}^{2}+\gamma_{n}\right)}
$$

Similarly using the result of Fourier Transform in (5.25), the amplitude for the threepoint vertex $F_{5}^{\prime}$ can be written as

$$
\sum_{m, n} \int \frac{d l}{2 \pi} \frac{F_{5}^{\prime}(0, l, n) F_{5}^{\prime *}(0, l, n)}{\left(\omega_{m}^{2}+\lambda_{n}\right)\left(l^{2}+\omega_{m}^{2}\right)}=\sum_{m, n} \int \frac{d l}{2 \pi} \frac{e^{-\frac{l^{2}}{2}}}{\left(\omega_{m}^{2}+\lambda_{n}\right)\left(l^{2}+\omega_{m}^{2}\right)} \frac{\left(3 l^{(n+1)}+4 n(n-2) l^{(n-3)}\right)^{2}}{2^{(n+2)} \pi\left(4 n^{2}-2 n\right)(n-2) !} .
$$

After integrating the amplitude in (5.30) over $l$ and expanding about $n=\infty$ we get the expansion

$$
\begin{aligned}
& \sum_{m, n} \int \frac{d l}{2 \pi} \frac{F_{5}^{\prime}(0, l, n) F_{5}^{\prime *}(0, l, n)}{\left(\omega_{m}^{2}+\lambda_{n}\right)\left(l^{2}+\omega_{m}^{2}\right)} \\
= & \frac{1}{2} \sum_{m, n} \frac{1}{\left(\omega_{m}^{2}+\lambda_{n}\right)}\left[\left(\frac{2 \sqrt{2} \sqrt{\frac{1}{n}}}{\pi}+\mathcal{O}\left(\frac{1}{n}\right)^{1}\right)\right. \\
& \left.+2^{-n}\left(\frac{e}{n}\right)^{n}\left(\frac{1}{\omega_{m}^{2}}\right)^{-n} \sec (n \pi)\left(-\frac{e^{\frac{\omega_{m}^{2}}{2}} \sqrt{\frac{2}{\pi}} n^{7 / 2}}{\omega_{m}^{7}}+\mathcal{O}\left(\frac{1}{n}\right)^{3}\right)\right] .
\end{aligned}
$$

The terms with odd powers of $\omega_{m}$ vanishes under the sum over $m$ over $\{-\infty, \infty\}$. The leading order term in the expansion of the amplitude (5.31) that contributes to the UV divergence is

$$
\sum_{m, n} \frac{4}{2 \pi \sqrt{2 n}} \frac{1}{\left(\omega_{m}^{2}+\lambda_{n}\right)}
$$

As for the amplitude containing the three-point vertex $\tilde{F}_{5}^{\prime}(0, l, n)$ we have

$$
\begin{aligned}
& \sum_{m, n} \int \frac{d l}{2 \pi} \frac{\tilde{F}_{5}^{\prime}(0, l, n) \tilde{F}_{5}^{\prime *}(0, l, n)}{\omega_{m}^{2}\left(\omega_{m}^{2}+l^{2}\right)} \\
= & \sum_{m, n} \int \frac{d l}{2 \pi} \frac{e^{-\frac{l^{2}}{2}}}{\omega_{m}^{2}\left(\omega_{m}^{2}+l^{2}\right)} \frac{\left(3 l^{(n+1)}-4(n-1)(n-2) l^{(n-3)}\right)^{2}}{2^{(n+2)} \pi(4 n-2)(n-1) !} .
\end{aligned}
$$

We rewrite the propagators as

$$
\frac{1}{\omega_{m}^{2}\left(\omega_{m}^{2}+l^{2}\right)}=\frac{1}{l^{2}}\left(\frac{1}{\omega_{m}^{2}}-\frac{1}{\left(\omega_{m}^{2}+l^{2}\right)}\right)
$$

we thus have the following expression,

$$
\sum_{m} \int \frac{d l}{2 \pi} \frac{e^{-\frac{l^{2}}{2}}}{l^{2}}\left(\frac{1}{\omega_{m}^{2}}-\frac{1}{\omega_{m}^{2}+l^{2}}\right) \frac{\left(3 l^{(n+1)}-4(n-1)(n-2) l^{(n-3)}\right)^{2}}{2^{(n+2)} \pi(4 n-2)(n-1) !}
$$


In the first term of the above expression we perform the integral over $l$ and then take the $n \rightarrow \infty$ limit. The first term gives

$$
\sum_{m, n} \frac{1}{2 \pi \sqrt{2 n}} \frac{1}{\omega_{m}^{2}}
$$

In the second term we perform the sum over $n$, which gives

$$
-\sum_{m} \int \frac{d l}{2 \pi} \frac{e^{-\frac{l^{2}}{2}}}{l^{2}}\left(\frac{1}{\omega_{m}^{2}+l^{2}}\right) \frac{\left(-128+96 l^{4}-18 l^{8}-9 l^{10}+8 e^{\frac{l^{2}}{2}}\left(16-8 l^{2}-10 l^{4}+6 l^{6}+l^{8}\right)\right)}{16 l^{6}} .
$$

The divergent piece in the last line is

$$
-\frac{1}{2} \sum_{m} \int \frac{d l}{2 \pi \sqrt{q}}\left(\frac{1}{\omega_{m}^{2}+l^{2}}\right) .
$$

Let us now look at the fermionic amplitudes in (4.35). We first note that the massless fermionic fields namely $R_{i}^{3}$ and $L_{i}^{3}$ has propagators $\left(i \omega_{m} \pm l\right)^{-1}$ where the "+"-sign stands for $R_{i}^{3}$ and the "-"-sign for $L_{i}^{3}$. However while computing the two-point functions one needs to perform integrations with respect the momentum $l$ over the entire range of $\{-\infty, \infty\}$. Hence to analyze the UV behaviour it suffices to consider only one one sign for the propagators. For our purpose we consider the following propagator and decomposed it into

$$
\frac{1}{\left(i \omega_{m}-\sqrt{\lambda_{n}^{\prime}}\right)\left(i \omega_{m}+l\right)}=\frac{1}{2}\left(-\frac{1}{\omega_{m}^{2}+\lambda_{n}^{\prime}}-\frac{1}{\omega_{m}^{2}+l^{2}}+\frac{l^{2}+\lambda_{n}^{\prime}}{\left(\omega_{m}^{2}+\lambda_{n}^{\prime}\right)\left(\omega_{m}^{2}+l^{2}\right)}\right)
$$

where we have dropped the terms with odd powers of $\omega_{m}$ and $l$, because they are odd functions of $\omega_{m}$ and $l$ and will vanish with respect to sum over $m$ over $\{-\infty, \infty\}$ as well as integral over $l$ over the same interval. The fermionic vertices are all three-point vertices with contributions from the massless fermions $L_{i}^{3}$ and $R_{i}^{3}$ respectively in the loop. As found in the bosonic amplitude the integrals in (C.31), (C.32) and (C.34) at $k=0$ also amounts to computing the Fourier transform the Hermite polynomials from the massive fermions which in turn produce the vertex functions in terms of $l$ and $n$. The fermionic vertices an thus be written as

$$
\begin{aligned}
& F_{6}^{L}(0, n, l)=F_{7}^{L}(0, n, l)=(-1)^{n+1} i^{(n+1)} e^{-\frac{l^{2}}{4}} \frac{\left(\frac{l^{n}}{\sqrt{2 n}}-l^{(n-1)}\right)}{\sqrt{2 \sqrt{\pi}} \sqrt{2^{n+1} \sqrt{\pi}(n-1) !}} \\
& F_{6}^{R}(0, n, l)=F_{7}^{R}(0, n, l)=-(-1)^{n+1} i^{(n+1)} e^{-\frac{l^{2}}{4}} \frac{\left(\frac{l^{n}}{\sqrt{2 n}}+l^{(n-1)}\right)}{\sqrt{2 \sqrt{\pi}} \sqrt{2^{n+1} \sqrt{\pi}(n-1) !}} .
\end{aligned}
$$

Combining the equations (5.39) with (C.32), (C.31) and (C.34), the total fermion two-point functions for the tree-level tachyon at finite temperature is given by

$$
\begin{aligned}
& \Sigma^{3}(0,0,0,0, \beta, q) \\
= & (8) \sum_{m, n} \int \frac{d l}{2 \pi} \frac{1}{2}\left(-\frac{1}{\omega_{m}^{2}+\lambda_{n}^{\prime}}-\frac{1}{\omega_{m}^{2}+l^{2}}+\frac{l^{2}+\lambda_{n}^{\prime}}{\left(\omega_{m}^{2}+\lambda_{n}^{\prime}\right)\left(\omega_{m}^{2}+l^{2}\right)}\right) \\
& \left(e^{-\frac{l^{2}}{2}} \frac{2\left(\frac{l^{n}}{\sqrt{2 n}}-l^{(n-1)}\right)^{2}+2\left(\frac{l^{n}}{\sqrt{2 n}}+l^{(n-1)}\right)^{2}+4\left(\frac{l^{n}}{\sqrt{2 n}}-l^{(n-1)}\right)\left(\frac{l^{n}}{\sqrt{2 n}}+l^{(n-1)}\right)}{2^{n+2} \pi(n-1) !}\right) .
\end{aligned}
$$


Combining all the fermionic vertices, eq. (5.42) can be finally written down as

$$
\begin{aligned}
& \Sigma^{3}(0,0,0,0, \beta, q)=(8) \sum_{m, n} \int \frac{d l}{2 \pi} \frac{1}{2}\left[-\frac{1}{\omega_{m}^{2}+\lambda_{n}^{\prime}}\left(e^{-\frac{l^{2}}{2}} \frac{l^{2 n}}{2^{n+1} \pi n !}\right)\right. \\
& \left.-\frac{1}{\omega_{m}^{2}+l^{2}}\left(e^{-\frac{l^{2}}{2}} \frac{l^{2 n}}{2^{n+1} \pi n !}\right)+\frac{l^{2}+\lambda_{n}^{\prime}}{\left(\omega_{m}^{2}+\lambda_{n}^{\prime}\right)\left(\omega_{m}^{2}+l^{2}\right)}\left(e^{-\frac{l^{2}}{2}} \frac{l^{2 n}}{\left.2^{n+1} \pi n\right) !}\right)\right]
\end{aligned}
$$

The first term in (5.43) upon integration over the momentum $l$ and in the $n \rightarrow \infty$ limit yields the leading order fermionic contribution

$$
-\frac{1}{2}(16) \sum_{m, n} \frac{1}{2 \pi \sqrt{2 n}} \frac{1}{\omega_{m}^{2}+\lambda_{n}^{\prime}} .
$$

In the second term in (5.43) we sum over $n$ and then take the $l \rightarrow \infty$ limit and extract the leading order term as

$$
-\frac{1}{2}(8) \int \frac{d l}{2 \pi \sqrt{q}} \sum_{m} \frac{1}{\omega_{m}^{2}+l^{2}}
$$

In the 3rd and last term we integrate over $l$ and the leading order term in the large $n$-expansion is given by

$$
\frac{1}{2}(8) \sum_{m, n} \frac{4}{2 \pi \sqrt{2 n}} \frac{1}{\omega_{m}^{2}+\lambda_{n}^{\prime}}
$$

The total leading order contribution to the UV divergence from the bosonic side is

$$
\begin{aligned}
& \underbrace{\frac{1}{2} \sum_{m, n} \frac{1}{2 \pi \sqrt{2 n}} \frac{1}{\omega_{m}^{2}+\lambda_{n}}+\frac{1}{2} \sum_{m, n} \frac{7 \times 2}{2 \pi \sqrt{2 n}} \frac{1}{\omega_{m}^{2}+\gamma_{n}}}_{\text {amplitudes involving } F_{1}(0,0, n, n) \text { and } F_{2}(0,0, n, n)}+\underbrace{\sum_{m, n} \frac{1}{2 \pi \sqrt{2 n}} \frac{1}{2 \omega_{m}^{2}}}_{\tilde{F}_{1}(0,0, n, n)} \\
& -\underbrace{\int \frac{d l}{2 \pi \sqrt{q}} \sum_{m} \frac{1}{2 \omega_{m}^{2}}+\frac{1}{2} \sum_{m, n} \frac{1}{2 \pi \sqrt{2 n}} \frac{1}{\omega_{m}^{2}+\lambda_{n}}}_{\text {amplitude involving } F_{4}(0, l, n)}+\underbrace{\int \frac{d l}{2 \pi \sqrt{q}} \sum_{m} \frac{1}{2 \omega_{m}^{2}}}_{F_{3}(0,0, l,-l)} \\
& +\underbrace{\left(\frac{1}{2}(7) \int \frac{d l}{2 \pi \sqrt{q}} \sum_{m} \frac{1}{\omega_{m}^{2}+l^{2}}+\frac{1}{2} \times \frac{1}{2} \int \frac{d l}{2 \pi \sqrt{q}} \sum_{m} \frac{1}{\omega_{m}^{2}+l^{2}}\right)}_{\text {amplitudes involving } F_{2}^{\prime}(0,0, n, n) \text { and } F_{3}^{\prime}(0,0, n, n)} \\
& +\underbrace{\frac{1}{2} \times \frac{1}{2} \int \frac{d l}{2 \pi \sqrt{q}} \sum_{m} \frac{1}{\omega_{m}^{2}+l^{2}}-\sum_{m, n} \frac{1}{2 \pi \sqrt{2 n}} \frac{1}{2 \omega_{m}^{2}}}_{\text {amplitude involving } \tilde{F}_{5}^{\prime}(0, l, n)} \\
& -\underbrace{\left(\frac{1}{2}(7) \sum_{m, n} \frac{4}{2 \pi \sqrt{2 n}} \frac{1}{\omega_{m}^{2}+\gamma_{n}}+\frac{1}{2} \sum_{m, n} \frac{4}{2 \pi \sqrt{2 n}} \frac{1}{\omega_{m}^{2}+\lambda_{n}}\right)} .
\end{aligned}
$$


The total leading order contribution to the UV divergence from the amplitudes containing fermions in the loop is

$$
\begin{aligned}
& -\underbrace{\frac{1}{2}(16) \sum_{m, n} \frac{1}{2 \pi \sqrt{2 n}} \frac{1}{\omega_{m}^{2}+\lambda_{n}^{\prime}}}_{\text {1st term in } \Sigma^{3}(0,0,0,0, \beta, q)}-\underbrace{\frac{1}{2}(8) \int \frac{d l}{2 \pi \sqrt{q}} \sum_{m} \frac{1}{\omega_{m}^{2}+l^{2}}}_{\text {2nd term in } \Sigma^{3}(0,0,0,0, \beta, q)} \\
& +\underbrace{\frac{1}{2}(8) \sum_{m, n} \frac{4}{2 \pi \sqrt{2 n}} \frac{1}{\omega_{m}^{2}+\lambda_{n}^{\prime}}}_{\text {3rd term in } \Sigma^{3}(0,0,0,0, \beta, q)}
\end{aligned}
$$

As $n \rightarrow \infty$, we see that $\gamma_{n}=\lambda_{n}=\lambda_{n}^{\prime}=2 n q$. Comparing (5.47) with (5.48), we see that the leading order terms from bosonic sides cancel with that from the fermionic side. In this method of proving UV finiteness of the finite temperature corrections to the tree-level tachyon mass-squared the UV divergence in $l$ or $n$ is thus softened by the fact that the Matsubara sum is left untouched. The large $n$ expansion is valid under the assumption that $m<n$. This assumption restricts our proof to a corner in the phase space. However the counting of the degrees of freedom on the bosonic and fermionic sides still match which in turns forces the divergences to cancel out. We expect the finiteness of the two-point functions to hold for large values of $m$ also because higher order terms in the expansion is heavily suppressed by Gaussian factors.

\subsection{Infrared problem}

We now address the problem of infrared divergences. The appearance of IR divergence is due to the presence of massless fields namely $\tilde{C}_{w, k}, A_{x}^{3}$ and $\Phi_{I}^{3}$ (for $I=1 \cdots 8$ ) in the loop. To compute the IR-finite two-point $C_{w, k}$ amplitude we shall follow a two step procedure as mentioned in the introduction. In the first step we compute the temperature corrected masses-squared of the massless fields by integrating over the modes in the internal lines with an IR cutoff. The next step is to introduce these masses in the propagators for the massless fields. This is equivalent to summing over an infinite set of diagrams which is illustrated in figure 5. In the figure, the $\chi$ field stands for the modes with tree-level mass zero. The bold line is the corrected propagator for the $\chi$ field due to the sum of an infinite set of diagrams on the right.

At each temperature the sums and the integrals are now IR-finite because all fields in the loops are now massive. Thus we can compute the mass-squared corrections and obtain the temperature corrected effective masses-squared of the tree-level tachyon as a function of $q$ and $\beta$.

In the following few sections we compute the two-point functions for the massless modes of the doublet of fields $\left(\Phi_{1}^{1}, A_{x}^{2}\right)$ namely the $\tilde{C}_{w, k}^{\prime} s$ and the other massless fields namely $\Phi_{1}^{3}, \Phi_{I}^{3}, A_{x}^{3}$ at finite temperature.

\subsection{Two-point functions for the $\tilde{C}_{w, k}$ modes}

In this section we compute the two point function for the $\tilde{C}_{w, k}$ modes. During these computations we note that there are no normalizable eigenfunctions $\tilde{A}_{n}(x)$ and $\tilde{\phi}_{n}(x)$ (see eqn (2.24)) for $n=0$. 

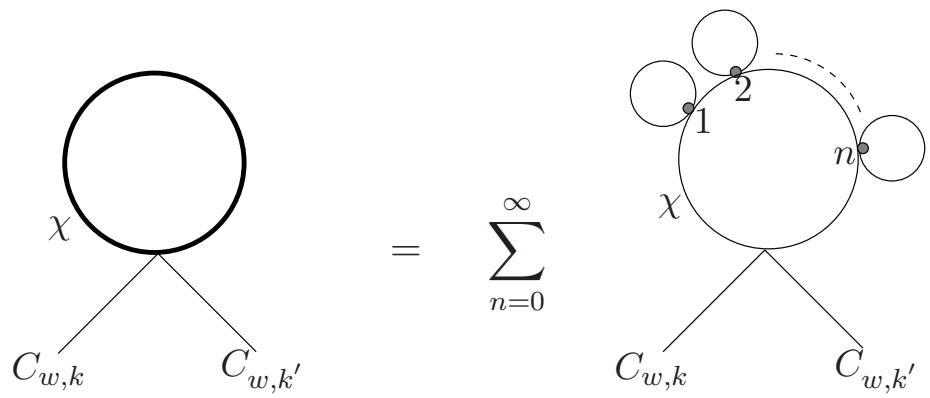

Figure 5. Diagram showing the correction to the propagator for the $\chi$ field due to mass insertions.

The amplitudes with bosons in the loop and consisting of the four-point vertices for the one-loop masses-squared of the massless modes are given by

$$
\begin{aligned}
\Sigma_{H}^{1}\left(w, w^{\prime}, k, k^{\prime}, \beta, q\right)=\frac{1}{2} N \sum_{m}\left[\sum_{n}\right. & \left(\frac{H_{1}\left(k, k^{\prime}, n, n\right)}{\omega_{m}^{2}+\lambda_{n}}+\frac{\tilde{H}_{1}\left(k, k^{\prime}, n, n\right)}{\omega_{m}^{2}}+\frac{7 H_{2}\left(k, k^{\prime}, n, n\right)}{\omega_{m}^{2}+\gamma_{n}}\right) \\
& +\int \frac{d l}{(2 \pi \sqrt{q})}\left(\frac{7 H_{2}^{\prime}\left(k, k^{\prime}, l,-l\right)}{\omega_{m}^{2}+l^{2}}+\frac{H_{3}^{\prime}\left(k, k^{\prime}, l,-l\right)}{\omega_{m}^{2}+l^{2}}\right) \\
& \left.+\int \frac{d l}{2 \pi \sqrt{q}} \frac{H_{3}\left(k, k^{\prime} l,-l\right)}{\omega_{m}^{2}}\right] \delta_{w+w^{\prime}}
\end{aligned}
$$

where $w=m+m^{\prime}$ '. Here ' $H$ ' denotes the vertices corresponding to the finite temperature two-point functions for the massless modes $\tilde{C}_{w, k}$ 's. The Feynman diagrams comprising the four-point vertices that contribute to the mass-squared corrections to the massless (at tree-level) modes $\tilde{C}_{w, k}$ are depicted in figure 6 .

The amplitudes arising from three-point interaction vertices for the massless modes $\tilde{C}_{w, k}$ are collected into

$$
\begin{aligned}
\Sigma_{H}^{2}\left(w, w^{\prime}, k, k^{\prime}, \beta, q\right)= & -\frac{1}{2} q N \sum_{m, n}\left[\int \frac{d l}{2 \pi \sqrt{q}} \frac{H_{4}(k, l, n) H_{4}^{*}\left(k^{\prime}, l, n\right)}{\left(\omega_{m}^{2}+\lambda_{n}\right) \omega_{m^{\prime}}^{2}}\right. \\
& +\int \frac{d l}{2 \pi \sqrt{q}} \frac{\tilde{H}_{4}(k, l, n) \tilde{H}_{4}^{*}\left(k^{\prime}, l, n\right)}{\omega_{m}^{2} \omega_{m^{\prime}}^{2}} \\
& +\int \frac{d l}{2 \pi \sqrt{q}}\left(\frac{7 H_{5}(k, l, n) H_{5}^{*}\left(k^{\prime},-l, n\right)}{\left(\omega_{m}^{2}+\gamma_{n}\right)\left(\omega_{m^{\prime}}^{2}+l^{2}\right)}+\frac{H_{5}^{\prime}(k, l, n) H_{5}^{\prime *}\left(k^{\prime},-l, n\right)}{\left(\omega_{m}^{2}+\lambda_{n}\right)\left(\omega_{m^{\prime}}^{2}+l^{2}\right)}\right) \\
& \left.+\int \frac{d l}{2 \pi \sqrt{q}} \frac{\tilde{H}_{5}^{\prime}(k, l, n) \tilde{H}_{5}^{\prime *}\left(k^{\prime},-l, n\right)}{\left(\omega_{m}^{2}\right)\left(\omega_{m^{\prime}}^{2}+l^{2}\right)}\right] \delta_{w+w^{\prime}}
\end{aligned}
$$

where $w=m+m^{\prime}$. The Feynman diagrams for the three-point interactions are given in figure 7. The various four-point and three-point vertices are given in appendix (D.1). Note that the one-loop mass-squared corrections to the massless modes have the same 


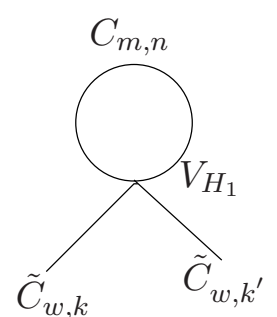

(a)

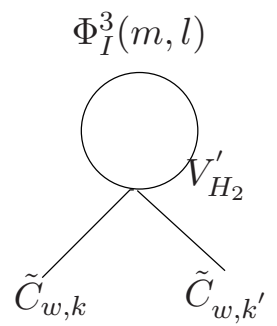

(d)

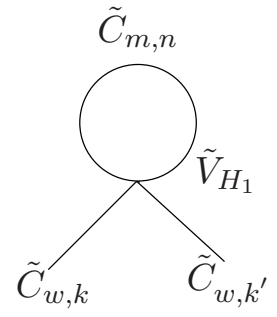

(b)

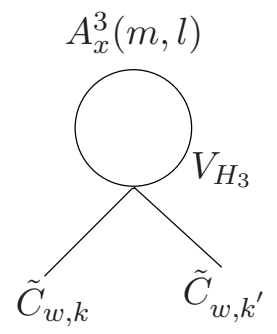

(e)

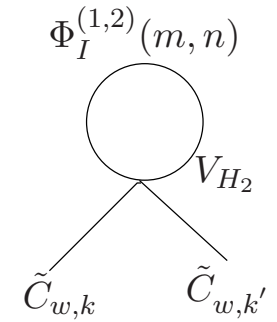

(c)

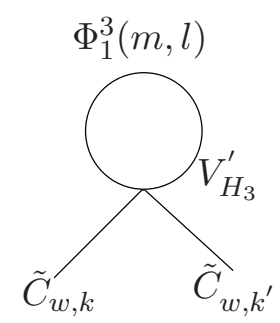

(f)

Figure 6. Feynman diagrams for the two-point $\tilde{C}_{w, k}$ amplitudes with four-point vertices.

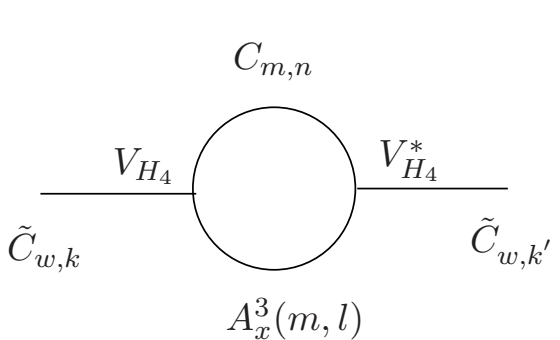

(a)

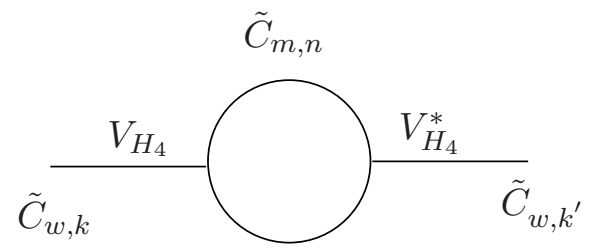

$A_{x}^{3}(m, l)$

$\Phi_{I}^{(1,2)}(m, n)$

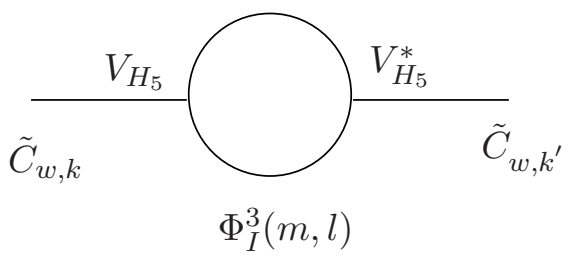

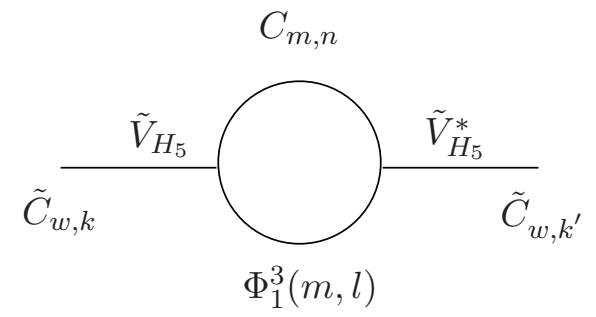

(d) (c)

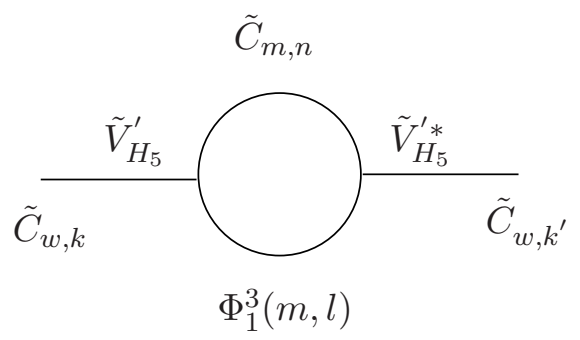

(e)

Figure 7. Feynman diagrams for the two-point $\tilde{C}_{w, k}$ amplitudes with three-point vertices. 
structure as the tachyonic amplitudes. This is because they originate from the same set of interactions for the doublet $\zeta(x, \tau)$ in the action. The only difference between (4.5), (4.6) and (5.49), (5.50) is that the momentum modes in the external legs of the Feynman diagrams for the latter are now the massless modes $\tilde{C}_{w, k}$. Upon performing the Matsubara sums, the bosonic amplitudes namely (5.49) and (5.50) can be recast as

$$
\begin{aligned}
\Sigma_{H}^{1}\left(0,0, k, k^{\prime}, \beta, q\right)=\frac{1}{2}[ & \sum_{n} \frac{H_{1}\left(k, k^{\prime}, n, n\right)}{\sqrt{(2 n-1)}}\left(\frac{1}{2}+\frac{1}{e^{\beta \sqrt{(2 n-1) q}}-1}\right) \\
& +N \sum_{m}\left(\sum_{n} \frac{\tilde{H}_{1}\left(k, k^{\prime}, n, n\right)}{\omega_{m}^{2}}+\int \frac{d l}{2 \pi \sqrt{q}} \frac{H_{3}\left(k, k^{\prime}, l-l\right)}{\omega_{m}^{2}}\right) \\
& +\sum_{n}\left(\frac{7 H_{2}\left(k, k^{\prime}, n, n\right)}{\sqrt{(2 n+1)}}\left(\frac{1}{2}+\frac{1}{e^{\beta \sqrt{(2 n+1) q}}-1}\right)\right) \\
& \left.+\left(\int \frac{d l}{2 \pi \sqrt{q}} \frac{(7+1 / 2) \delta_{k, k^{\prime}}}{(l / \sqrt{q})}\left(\frac{1}{2}+\frac{1}{e^{\beta l}-1}\right)\right)\right]
\end{aligned}
$$

$$
\begin{aligned}
& \Sigma_{H}^{2}\left(k, k^{\prime}, \beta, q\right) \\
&=-\frac{1}{2} \sum_{n}\left[\int \frac{d l}{2 \pi \sqrt{q}} \frac{H_{4}(k, l, n) H_{4}^{*}\left(k^{\prime}, l, n\right)}{(2 n-1)}\left[\left(\sum_{m} \frac{\sqrt{q}}{\beta \omega_{m}^{2}}-\frac{1}{\sqrt{2 n-1}}\left(\frac{1}{2}+\frac{1}{e^{\sqrt{(2 n-1) q} \beta}}-1\right)\right)\right]\right. \\
&+q N \int \frac{d l}{2 \pi \sqrt{q}} \sum_{m} \frac{\tilde{H}_{4}(k, l, n) \tilde{H}_{4}^{*}\left(k^{\prime}, l, n\right)}{\omega_{m}^{4}} \\
&+\int \frac{d l}{2 \pi \sqrt{q}}\left[\frac { 7 H _ { 5 } ( k , l , n ) H _ { 5 } ^ { * } ( k ^ { \prime } , - l , n ) } { ( l / \sqrt { q } ) ^ { 2 } - ( 2 n + 1 ) } \left(\frac{1}{\sqrt{2 n+1}}\left(\frac{1}{2}+\frac{1}{e^{\sqrt{(2 n+1) q} \beta}-1}\right)\right.\right. \\
&\left.\left.-\frac{1}{(l / \sqrt{q})}\left(\frac{1}{2}+\frac{1}{e^{l \beta}-1}\right)\right)\right] \\
&+\int \frac{d l}{2 \pi \sqrt{q}}\left[\frac { H _ { 5 } ^ { \prime } ( k , l , n ) H _ { 5 } ^ { \prime * } ( k ^ { \prime } , - l , n ) } { ( l / \sqrt { q } ) ^ { 2 } - ( 2 n - 1 ) } \left(\frac{1}{\sqrt{2 n-1}}\left(\frac{1}{2}+\frac{1}{e^{\sqrt{(2 n-1) q} \beta}-1}\right)\right.\right. \\
&\left.\left.-\frac{1}{(l / \sqrt{q})}\left(\frac{1}{2}+\frac{1}{e^{l \beta}-1}\right)\right)\right] \\
&\left.+\int \frac{d l}{2 \pi \sqrt{q}} \frac{\tilde{H}_{5}^{\prime}(k, l, n) \tilde{H}_{5}^{\prime *}\left(k^{\prime},-l, n\right)}{(l / \sqrt{q})^{2}}\left(\sum_{m} \frac{1}{\omega_{m}^{2}}-\frac{1}{(l / \sqrt{q})}\left(\frac{1}{2}+\frac{1}{e^{l \beta}-1}\right)\right)\right] .
\end{aligned}
$$

respectively. Similarly using the various vertex functions given in appendix (D.2), the finitetemperature contribution due to fermions in the loop to the mass-squared corrections for the massless modes is given by equation (5.53). The relevant Feynman diagrams are listed in figure 8 and figure 9 .

$$
\begin{aligned}
\Sigma_{H}^{3}\left(w, w^{\prime}, k, k^{\prime}, \beta, q\right)= & -(8 N) \sum_{n, m, m^{\prime}} \int \frac{d l}{2 \pi \sqrt{q}} \frac{1}{\left(i \omega_{m}-\sqrt{\lambda_{n}^{\prime}}\right)} \\
& \times\left[\frac{H_{6}^{R}(k, n, l) H_{6}^{R *}\left(k^{\prime}, n, l\right)}{\left(i \omega_{m^{\prime}}+l\right)}+\frac{H_{6}^{L}(k, n, l) H_{6}^{L *}\left(k^{\prime}, n, l\right)}{\left(i \omega_{m^{\prime}}-l\right)}\right.
\end{aligned}
$$




$$
\begin{aligned}
& +\frac{H_{7}^{L}(k, n, l) F_{7}^{H *}\left(k^{\prime}, n, l\right)}{\left(i \omega_{m^{\prime}}+l\right)}+\frac{H_{7}^{R}(k, n, l) H_{7}^{R *}\left(k^{\prime}, n, l\right)}{\left(i \omega_{m^{\prime}}-l\right)} \\
& +\frac{H_{6}^{R}(k, n, l) H_{7}^{L *}\left(k^{\prime}, n, l\right)+H_{6}^{R *}(k, n, l) H_{7}^{L}\left(k^{\prime}, n, l\right)}{\left(i \omega_{m^{\prime}}+l\right)} \\
& \left.+\frac{H_{6}^{L}(k, n, l) H_{7}^{R *}\left(k^{\prime}, n, l\right)+H_{6}^{L *}(k, n, l) H_{7}^{R}\left(k^{\prime}, n, l\right)}{\left(i \omega_{m^{\prime}}-l\right)}\right] \delta_{w+w^{\prime}}
\end{aligned}
$$

where $w=m+m^{\prime}$. After performing the Matsubara sums the amplitude (5.53) assumes the following form.

$$
\begin{aligned}
\Sigma_{H}^{3}\left(0,0, k, k^{\prime}, \beta, q\right)= \\
(8 N) \sum_{n}\left[\int \frac{d l}{2 \pi \sqrt{q}}\left(\frac{-\beta \tanh \left(\frac{\beta l}{2}\right)-\beta \tanh \left(\frac{1}{2} \beta \sqrt{2 n q}\right)}{2(l+\sqrt{2 n q})}\right)\right. \\
\quad\left[H_{6}^{R}(k, n, l) H_{6}^{R *}\left(k^{\prime}, n, l\right)+H_{7}^{L}(k, n, l) H_{7}^{L *}\left(k^{\prime}, n, l\right)\right. \\
\left.+H_{6}^{R}(k, n, l) H_{7}^{L *}\left(k^{\prime}, n, l\right)+H_{6}^{R *}(k, n, l) H_{7}^{L}\left(k^{\prime}, n, l\right)\right] \\
+\int \frac{d l}{2 \pi \sqrt{q}}\left(\frac{-\beta \tanh \left(\frac{\beta l}{2}\right)+\beta \tanh \left(\frac{1}{2} \beta \sqrt{2 n q}\right)}{2(l-\sqrt{2 n q})}\right) \\
+\left[H_{6}^{L}(k, n, l) H_{6}^{L *}\left(k^{\prime}, n, l\right)+H_{7}^{R}(k, n, l) H_{7}^{R *}\left(k^{\prime}, n, l\right)\right. \\
\left.+H_{6}^{L}(k, n, l) H_{7}^{R *}\left(k^{\prime}, n, l\right)+H_{6}^{L *}(k, n, l) H_{7}^{R}\left(k^{\prime}, n, l\right)\right] .
\end{aligned}
$$

At this point we recall that there is no normalizable eigenfunction for the massless modes $\tilde{C}_{w, 0}$. Hence the counting starts from $k=1$. These massless modes appear as the fluctuations $\tilde{C}_{m, n}$ with $k$ replaced as $n$ in the one-loop diagrams for the tree-level tachyon (see figures 1 and 2) where we need to sum over all $n$. As mentioned before the two point functions for all the $C_{w, k}$ and the $\tilde{C}_{w, k}$ modes are coupled to each other at the one loop level giving rise to an infinite dimensional mass-matrix. To get the corrected spectrum we must re-diagonalize the mass matrix. Since our approach is to get the final finite values of the masses-squared numerically, for simplicity we shall work with a finite dimensional matrix

for the $\tilde{C}_{w, k}$ modes. Like the two point amplitude for the $C_{w, k}$, the two-point functions of the massless modes also have contributions from the massless fields $\Phi_{1}^{3}, \Phi_{I}^{3} ; I \neq 1$ and $A_{x}^{3}$ in the loop hence has the problem of infrared divergence and will be addressed in the way as mentioned before. The UV finiteness of the amplitudes for the two-point functions of the fields $\tilde{C}_{w, k}$ can be checked using the method used for the tachyonic case.

\subsection{Two point function for $\Phi_{1}^{3}$}

Using the vertices computed in appendix E.1 we first write down the two point amplitude for $\Phi_{1}^{3}$. The Feynman diagrams involving the four-point vertices is depicted in figure 10 .

The one-loop two-point functions involving the four-point vertices given in section E.1 contributing to the finite-temperature mass-squared corrections to the tree-level massless 


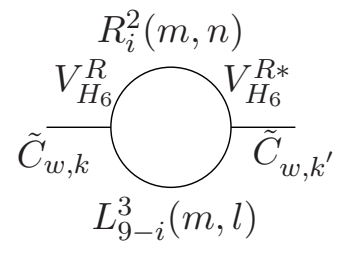

(a)

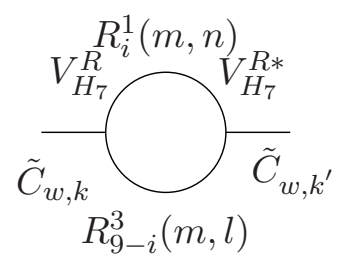

(c)

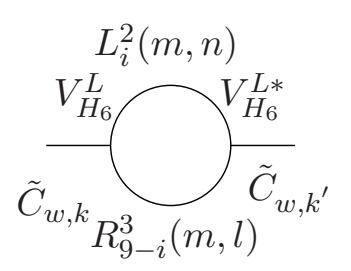

(b)

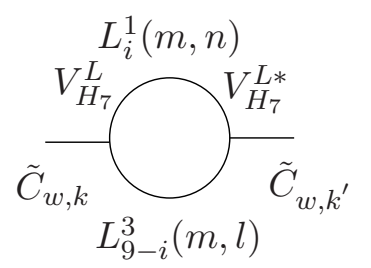

(d)

Figure 8. Feynman diagrams for the amplitudes with three-point fermionic vertices $V_{H}^{R / L}$ and their complex conjugates $V_{H}^{* R / L}$.

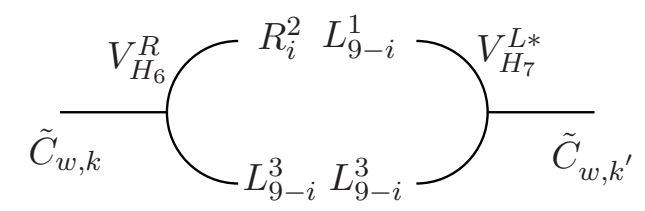

(a)

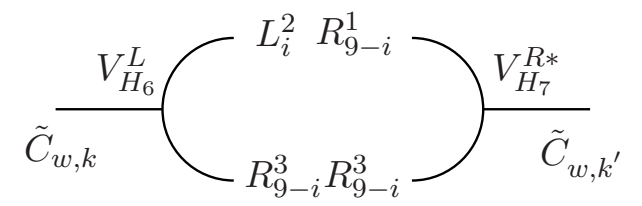

(b)

Figure 9. Feynman diagrams showing the cross terms in the amplitude with fermions in the loop.

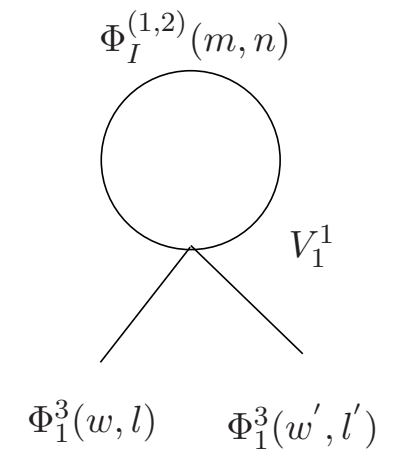

(a)

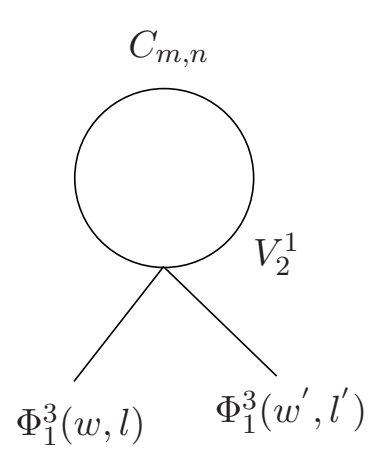

(b)

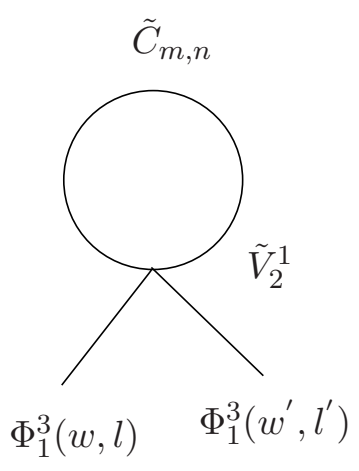

(c)

Figure 10. Feynman diagrams for the amplitudes with four-point bosonic vertices $V_{1}^{1}, V_{2}^{1}, \tilde{V}_{2}^{1}$. 


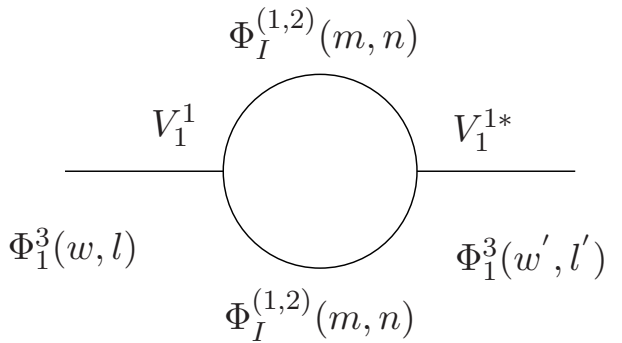

(a)

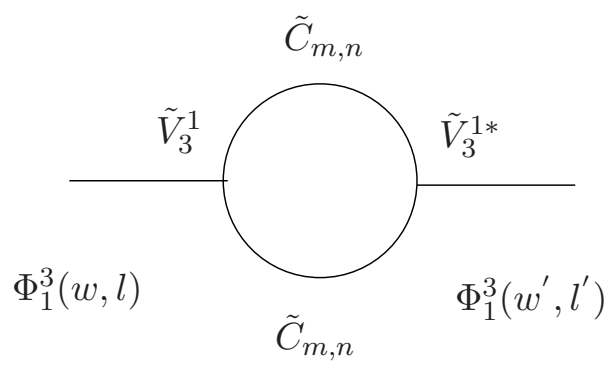

(c)

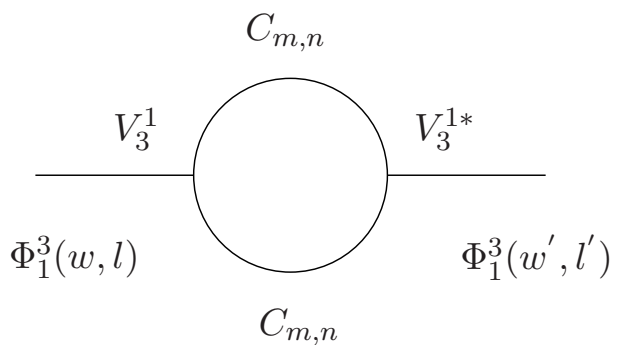

(b)

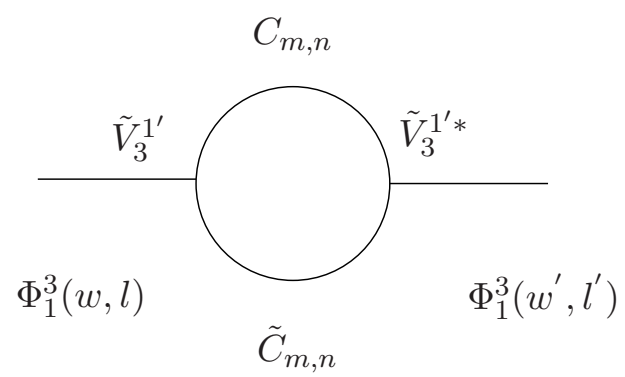

(d)

Figure 11. Feynman diagrams with three-point bosonic vertices $V_{1}^{1^{\prime}}, V_{3}^{1}, \tilde{V}_{3}^{1}, \tilde{V}_{3}^{1^{\prime}}$.

field $\Phi_{1}^{3}$ can be collected into

$$
\Sigma_{\Phi_{1}^{3}-\Phi_{1}^{3}}^{1}=\frac{1}{2} N \sum_{m, n}\left[(7 \times 2) \frac{G_{1}^{1}\left(l, l^{\prime}, n, n\right)}{\left(\omega_{m}^{2}+\gamma_{n}\right)}+(2) \frac{G_{2}^{1}\left(l, l^{\prime}, n, n\right)}{\left(\omega_{m}^{2}+\lambda_{n}\right)}+(2) \frac{\tilde{G}_{2}^{1}\left(l, l^{\prime}, n, n\right)}{\left(\omega_{m}^{2}\right)}\right] \delta_{w+w^{\prime}}
$$

The first term in (5.55) has contributions from the massive fields $\Phi_{I}^{(1,2)}, I \neq 1$ in the loop with four-point vertex and propagator given in (C.3) and the corresponding Feynman diagram is given by figure 10 (a). The second term bears contributions from the fields $C_{m, n}$ with propagator (C.1) and depicted in the Feynman diagram in figure 10(b). The third term involves the massless fields $\tilde{C}_{m, n}$ in the loop with propagator (C.2) and corresponding Feynman diagram in figure 10(c). Similarly the three-point bosonic interactions contributing to the finite temperature corrections to the massless field $\Phi_{1}^{3}(w, l)$ are collected in the mass-squared correction (5.56). The corresponding Feynman diagrams are given in figure 11.

$$
\begin{aligned}
\Sigma_{\Phi_{1}^{3}-\Phi_{1}^{3}}^{2}= & -\frac{1}{2} q N \sum_{m, n, n^{\prime}}\left[(7 \times 2) \frac{G_{1}^{1^{\prime}}\left(l, l^{\prime}, n, n\right)}{\left(\omega_{m}^{2}+\gamma_{n}\right)\left(\omega_{m^{\prime}}^{2}+\gamma_{n^{\prime}}^{\prime}\right)}+(2) \frac{G_{3}^{1}\left(l, n, n^{\prime}\right) G_{3}^{1}\left(l^{\prime}, n, n^{\prime}\right)}{\left(\omega_{m}^{2}+\lambda_{n}\right)\left(\omega_{m^{\prime}}^{2}+\lambda_{n^{\prime}}\right)}\right. \\
& \left.+(2) \frac{\tilde{G}_{3}^{1}\left(l, n, n^{\prime}\right) \tilde{G}_{3}^{1}\left(l^{\prime}, n, n^{\prime}\right)}{\left(\omega_{m}^{2}\right)\left(\omega_{m^{\prime}}^{2}\right)}+(2) \frac{\tilde{G}_{3}^{1^{\prime}}\left(l, n, n^{\prime}\right) \tilde{G}_{3}^{1^{\prime}}\left(l^{\prime}, n, n^{\prime}\right)}{\omega_{m}^{2}\left(\omega_{m^{\prime}}^{2}+\lambda_{n^{\prime}}\right)}\right] \delta_{w+w^{\prime}}
\end{aligned}
$$

where $w=m^{\prime}+m$. The first term in (5.56) comprising the three-point vertex $G_{1}^{1^{\prime}}\left(l, n, n^{\prime}\right)$ involves the fields $\Phi_{I}^{(1,2)}, I \neq 1$ in the loops and is represented by the Feynman diagram in 


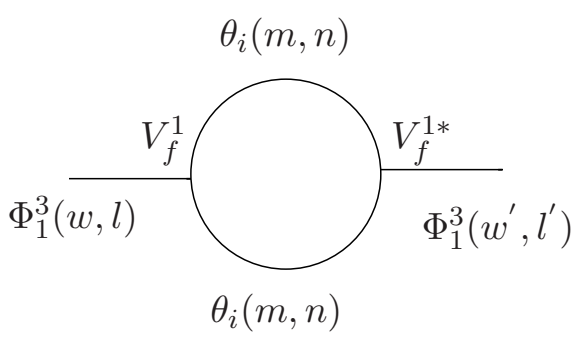

(a)

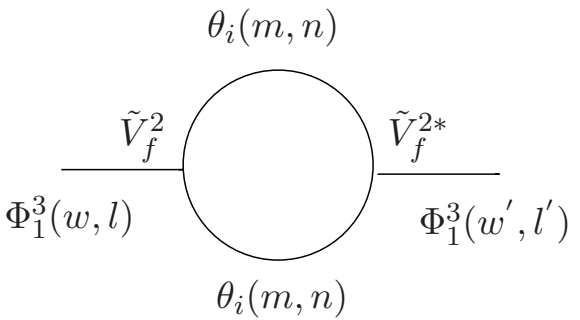

(b)

Figure 12. Feynman diagrams involving three-point vertices $V_{f}^{1}, V_{f}^{2}$.

figure 11(a). Similarly the second term in (5.56) involving the vertex $G_{3}^{1}\left(l, n, n^{\prime}\right)$ comprises of the fields $C_{m, n} \mathrm{~s}$ in the loop. The corresponding Feynman diagram is given in figure 10(b). The third term has contributions from $\tilde{C}_{m, n} \mathrm{~s}$ with Feynman diagram in figure 10(c), while the fourth term represented in figure 10(d) has contributions from the fields $C_{m, n}$ and $\tilde{C}_{m, n}$.

Figure 12 shows the amplitude involving the fermions in the loop. The corresponding expression is

$$
\begin{aligned}
\Sigma_{\Phi_{1}^{3}-\Phi_{1}^{3}}^{3}=\frac{1}{2} N \sum_{m, n, n^{\prime}} & {\left[(16 \times 2) \frac{G_{f}^{1}\left(l, n, n^{\prime}\right) G_{f}^{1 *}\left(l^{\prime}, n, n^{\prime}\right)}{\left(i \omega_{m}-\sqrt{\lambda_{n}^{\prime}}\right)\left(i \omega_{m^{\prime}}-\sqrt{\lambda_{n^{\prime}}^{\prime}}\right)}\right.} \\
& \left.-(16) \frac{G_{f}^{2}\left(l, n, n^{\prime}\right) G_{f}^{2 *}\left(l^{\prime}, n, n^{\prime}\right)}{\left(i \omega_{m}-\sqrt{\lambda_{n}^{\prime}}\right)\left(-i \omega_{m^{\prime}}-\sqrt{\lambda_{n^{\prime}}^{\prime}}\right)}\right] \delta_{w+w^{\prime}}
\end{aligned}
$$

with $w=m^{\prime}+m$. The first and the second term in (5.57) are depicted in the Feynman diagrams given in figures 12 (a) and 12(b) respectively. The various vertices given in section E.1 that are involved in the two-point functions realizing the mass-squared corrections to the tree-level massless field $\Phi_{1}^{3}$ can be exactly computed using the orthogonality relation for Hermite Polynomials. Setting the external momenta $l=l^{\prime}=w=w^{\prime}=0$, we can write down the various vertices as

$$
\begin{array}{rlrl}
G_{1}^{1}\left[0,0, n, n^{\prime}\right] & =\delta_{n, n^{\prime}}, & G_{2}^{1}\left[0,0, n, n^{\prime}\right] & =\frac{1}{2} \delta_{n, n^{\prime}}, \quad \tilde{G}_{2}^{1}\left[0,0, n, n^{\prime}\right]=\frac{1}{2} \delta_{n, n^{\prime}}, \\
G_{1}^{1^{\prime}}\left[0, n, n^{\prime}\right] & =\sqrt{2 n} \delta_{n-1, n^{\prime}}, & G_{3}^{1}\left[0, n, n^{\prime}\right] & =2 \sqrt{\frac{2 n(n-1)(n-2)}{(2 n-1)(2 n-3)}} \delta_{n-1, n^{\prime}}, \\
\tilde{G}_{3}^{1}\left[0, n, n^{\prime}\right] & =0 \\
\tilde{G}_{3}^{1^{\prime}}\left[0, n, n^{\prime}\right] & =-\left(\frac{\sqrt{2(n-1)}(n+1)}{\sqrt{(2 n-1)(2 n+1)}} \delta_{n+1, n^{\prime}}-\frac{\sqrt{2 n}(n-1)}{\sqrt{(2 n-1)(2 n-3)}} \delta_{n-1, n^{\prime}}\right) \\
G_{f}^{1}\left[0, n, n^{\prime}\right] & =-\frac{i}{4}\left(2 \delta_{n-1, n^{\prime}}\right), \quad G_{f}^{2}\left[0, n, n^{\prime}\right]=-\frac{i}{2}\left(\delta_{n+1, n},+\delta_{n-1, n^{\prime}}\right) .
\end{array}
$$

To prove the ultraviolet finiteness of the mass-squared corrections (5.55), (5.56) and (5.57), we set the external momenta $\left(l, l^{\prime}, w\right)=0$ and compute the vertices in the 
large $n$ limit. The vertices can be evaluated using the orthogonality condition for Hermite polynomials. The various four-point vertices given in (5.58) in the large $n$ limit assume the forms

$$
G_{1}^{1}(0,0, n, n)=1 ; \quad G_{2}^{1}(0,0, n, n)=\tilde{G}_{2}^{1}(0,0, n, n)=\frac{1}{2}
$$

where we have used the Kronecker delta's to set $n=n^{\prime}$. The three-point vertices given in (5.59) in the large $n$ limit become

$$
G_{1}^{1^{\prime}}\left(0, n, n^{\prime}\right) \sim \frac{\sqrt{2 n}}{2}\left[2 \delta_{n^{\prime}, n-1}\right] ; \quad G_{3}^{1}\left(0, n, n^{\prime}\right) \sim \frac{\sqrt{2 n}}{8}\left[8 \delta_{n^{\prime}, n-1}\right] .
$$

The three-point bosonic vertex $\tilde{G}_{3}^{1}\left(0, n, n^{\prime}\right)$ is found to be identically zero for all values $n$ in (5.60). Moreover the fermionic three-point vertices are exact for all values $n$. Hence the remaining three-point bosonic vertex (5.61) in the limit $n \rightarrow \infty$ can be written as

$$
\tilde{G}_{3}^{1^{\prime}}\left(0, n, n^{\prime}\right) \sim \frac{\sqrt{2 n}}{8}\left[4 \delta_{n^{\prime}, n-1}-4 \delta_{n^{\prime}, n+1}\right] .
$$

The ultraviolet contribution to the amplitude can now be written down by putting these asymptotic values of the vertices into (5.55), (5.56), (5.57). We get the following from the bosonic fields in the loop

$$
\begin{aligned}
& \Sigma_{\Phi_{1}^{3}-\Phi_{1}^{3}}^{1} \sim \frac{1}{2} N \sum_{m, n}\left[(7 \times 2) \frac{1}{\left(\omega_{m}^{2}+\gamma_{n}\right)}+(2) \frac{1 / 2}{\left(\omega_{m}^{2}+\lambda_{n}\right)}+(2) \frac{1 / 2}{\left(\omega_{m}^{2}\right)}\right] \\
& \Sigma_{\Phi_{1}^{3}-\Phi_{1}^{3}}^{2} \sim-\frac{1}{2} q N \sum_{m, n}\left[(7 \times 2) \frac{2 n}{\left(\omega_{m}^{2}+\gamma_{n}\right)^{2}}+(2) \frac{2 n}{\left(\omega_{m}^{2}+\lambda_{n}\right)^{2}}+(2) \frac{(2 n) / 2}{\omega_{m}^{2}\left(\omega_{m}^{2}+\lambda_{n}\right)}\right] .
\end{aligned}
$$

Noting that in the large $n$ limit, $\lambda_{n}=\gamma_{n} \sim 2 n q$,

$$
\begin{aligned}
\Sigma_{\Phi_{1}^{3}-\Phi_{1}^{3}}^{1}+\Sigma_{\Phi_{1}^{3}-\Phi_{1}^{3}}^{2} & \sim N \sum_{m, n}\left[(8) \frac{1}{\left(\omega_{m}^{2}+2 n q\right)}-(8) \frac{2 n q}{\left(\omega_{m}^{2}+2 n q\right)^{2}}\right] \\
& \sim \sum_{n} \frac{2}{\sqrt{2 n}} .
\end{aligned}
$$

In the last line we have done the sum over the Matsubara frequencies $m$ and omitted all the finite temperature dependent pieces. Similarly the asymptotic form of the fermionic contribution is,

$$
\Sigma_{\Phi_{1}^{3}-\Phi_{1}^{3}}^{3} \sim-N \sum_{m, n}\left[(4) \frac{1}{\left(\omega_{m}^{2}+\lambda_{n}^{\prime}\right)}-(4) \frac{1}{\left(i \omega_{m}-\sqrt{\lambda_{n}^{\prime}}\right)^{2}}\right] \sim-\sum_{n} \frac{2}{\sqrt{2 n}} .
$$

Thus the one-loop $\Phi_{1}^{3}-\Phi_{1}^{3}$ amplitude is ultraviolet finite.

One can exactly compute the various amplitudes in (5.55), (5.56) and (5.57) using the various vertices presented in (5.58)-(5.62) and their corresponding propagators (see appendix $(F)$ ). In particular one can write down the effective mass-squared for the field $\Phi_{1}^{3}$ as a function of $q$ and $\beta$ as

$$
m_{\Phi_{1}^{3}}^{2}(q, \beta)=m_{10}^{2}+m_{1}^{2}(q, \beta)
$$


where $m_{10}^{2}$ denotes the zero temperature quantum corrections which is made dimensionless by dividing the physical $m_{10}^{2}$ by $g^{2}$ and $m_{1}^{2}(q, \beta)$ denotes the temperature dependent masssquared corrections for the massless field $\Phi_{1}^{3}$. The zero temperature quantum corrections for all $n$ can be written as

$$
\begin{aligned}
m_{10}^{2}= & {\left[\sum_{n=0}^{\infty} \frac{7}{2 \sqrt{2 n+1}}+\sum_{n=2}^{\infty} \frac{1}{4 \sqrt{2 n-1}}-\frac{7}{2} \sum_{n=1}^{\infty}\left(\frac{n}{\sqrt{2 n-1}}-\frac{n}{\sqrt{2 n+1}}\right)\right.} \\
& \left.+\sum_{n=2}^{\infty}\left(\frac{(n-1)}{(2 n-1)^{\frac{5}{2}}}\left(\frac{(n+1)^{2}}{(2 n+1)}+\frac{n(n-1)}{(2 n-3)}\right)-2 \frac{n(n-1)(n-2)}{(2 n-1)(2 n-3)}\left(\frac{1}{\sqrt{2 n-3}}-\frac{1}{\sqrt{2 n-1}}\right)\right)\right] \\
& -\sum_{n=1}^{\infty} \frac{4}{\sqrt{2 n}+\sqrt{2 n-2}} .
\end{aligned}
$$

The zero temperature quantum correction given by (5.72) can be evaluated numerically. The convergent value is given by

$$
m_{10}^{2}=1.579
$$

The temperature dependent part in (5.71) can be written as

$$
\begin{aligned}
& m_{1}^{2}(q, \beta)=\left[\sum_{n=0}^{\infty} \frac{7}{\sqrt{2 n+1}} \frac{1}{\left(e^{\sqrt{(2 n+1) q} \beta}-1\right)}+\sum_{n=2}^{\infty} \frac{1}{2 \sqrt{2 n-1}} \frac{1}{\left(e^{\sqrt{(2 n-1) q} \beta}-1\right)}\right. \\
& -7 \sum_{n=1}^{\infty}\left(\frac{n}{\sqrt{2 n-1}} \frac{1}{\left(e^{\sqrt{(2 n-1) q} \beta}-1\right)}-\frac{n}{\sqrt{2 n+1}} \frac{1}{\left(e^{\sqrt{(2 n+1) q} \beta}-1\right)}\right) \\
& -4 \sum_{n=1}^{\infty} \frac{n(n-1)(n-2)}{(2 n-1)(2 n-3)}\left(\frac{1}{\sqrt{2 n-3}} \frac{1}{\left(e^{\sqrt{(2 n-3) q} \beta}-1\right)}-\frac{1}{\sqrt{2 n-1}} \frac{1}{\left(e^{\sqrt{(2 n-1) q} \beta}-1\right)}\right) \\
& +2 \sum_{n=2}^{\infty}\left(\frac{(n-1)}{(2 n-1)^{\frac{5}{2}}}\left(\frac{(n+1)^{2}}{(2 n+1)}+\frac{n(n-1)}{2 n-3}\right) \frac{1}{\left(e^{\sqrt{(2 n-1) q} \beta}-1\right)}\right) \\
& \left.+\left(\frac{1}{2}-2 \sum_{n=2}^{\infty}\left(\frac{(n-1)}{(2 n-1)}\left(\frac{(n+1)^{2}}{(2 n+1)^{2}}+\frac{n(n-1)}{(2 n-3)^{2}}\right)\right)\right) \sum_{m=-\infty}^{\infty} \frac{\sqrt{q} \beta}{4 \pi^{2} m^{2}}\right] \\
& +\left[\sum _ { n = 1 } ^ { \infty } \left(\left(4 \frac{1}{(\sqrt{2 n}+\sqrt{2(n-1)})}+2 \frac{(\sqrt{2(n+1)}+\sqrt{2(n-1)}-2 \sqrt{2 n})}{(\sqrt{2 n}-\sqrt{2(n-1)})(\sqrt{2(n+1)}-\sqrt{2 n})}\right) \frac{1}{\left(e^{\sqrt{2 n q} \beta}+1\right)}\right.\right. \\
& +\left(4 \frac{1}{(\sqrt{2 n}+\sqrt{2(n-1)})}-\frac{2}{\sqrt{2 n}-\sqrt{2(n-1)}}\right) \frac{1}{\left(e^{\sqrt{2(n-1) q}+1)}\right.} \\
& \left.\left.+\frac{2}{\sqrt{2(n+1)}-\sqrt{2 n}} \frac{1}{\left(e^{\sqrt{2(n+1) q} \beta}+1\right)}\right)\right] \text {. }
\end{aligned}
$$

\subsection{Two point function for $\Phi_{I}^{3}(I \neq 1)$}

Let us now write down all the amplitudes that constitute the finite temperature one-loop mass-squared corrections to the tree-level massless fields $\Phi_{I}^{3}(m, l)$, where $I \neq 1$. The computation of the vertices involving the fermions in this section has been done explicitly 


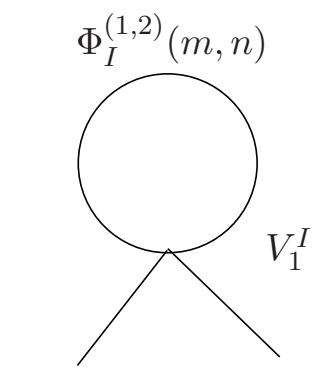

$\Phi_{I}^{3}(w, l) \quad \Phi_{I}^{3}\left(w^{\prime}, l^{\prime}\right)$

(a)

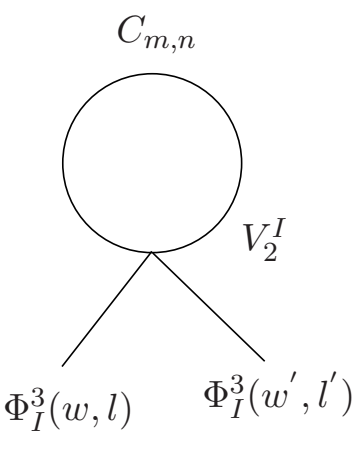

(b)

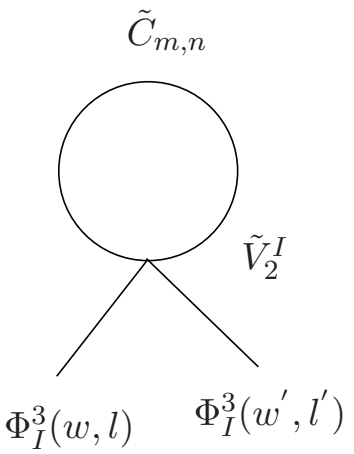

(c)

Figure 13. Feynman diagrams with four-point vertices $V_{1}^{I}, V_{2}^{I}, \tilde{V}_{2}^{I}$.

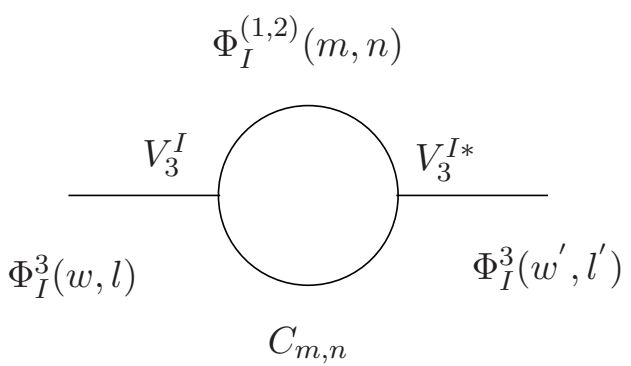

(a)

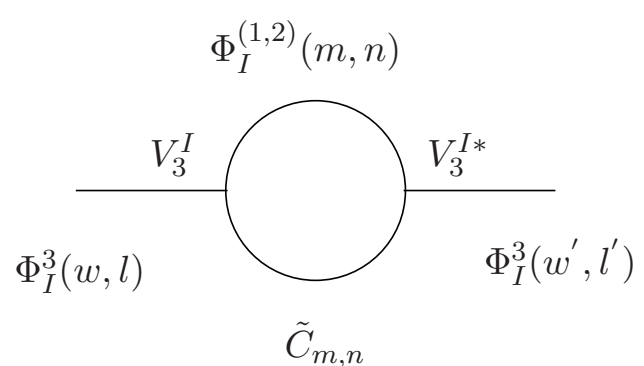

(b)

Figure 14. Feynman diagrams with the three-point vertices $V_{3}^{I}, \tilde{V}_{2}^{I}$.

for $I=2$. However the $\mathrm{SO}(7)$ invariance of the theory implies that the two point function is the same for all $I=2 \cdots 8$. Using the vertices listed in appendix E.2 we write below the expressions for the two point function. The Feynman diagrams involving the four-point bosonic interactions are depicted in the figure 13.

$$
\Sigma_{\Phi_{I}^{3}-\Phi_{I}^{3}}^{1}=\frac{1}{2} N \sum_{m, n}\left[(6 \times 2) \frac{G_{1}^{I}\left(l, l^{\prime}, n, n\right)}{\left(\omega_{m}^{2}+\gamma_{n}\right)}+(2) \frac{G_{2}^{I}\left(l, l^{\prime}, n, n\right)}{\left(\omega_{m}^{2}+\lambda_{n}\right)}+(2) \frac{\tilde{G}_{2}^{I}\left(l, l^{\prime}, n, n\right)}{\omega_{m}^{2}}\right] \delta_{w+w^{\prime}}
$$

The three terms in (5.75) are represented by the Feynman diagrams in figures 13(a), 13 (b) and 13(c) in the same order. The first term involves the fields $\Phi_{I}^{(1,2)}, I \neq 1$, the second term involves the fields $C_{m, n}$ and the third term comprises of the fields $\tilde{C}_{m, n}$. Similarly the three-point bosonic interactions of $\Phi_{I}^{3}(m, l)$ are represented in the Feynman diagrams in figure 14. The two-point function involving the bosonic three-point vertices and contributing to the one-loop finite temperature mass-corrections for the field $\Phi_{I}^{3}, I \neq 1$ is given by

$$
\Sigma_{\Phi_{I}^{3}-\Phi_{I}^{3}}^{2}=-\frac{1}{2} q N \sum_{m, n, n^{\prime}}\left[(2) \frac{G_{3}^{I}\left(l, n, n^{\prime}\right) G_{3}^{I}\left(l^{\prime}, n, n^{\prime}\right)}{\left(\omega_{m}^{2}+\gamma_{n}\right)\left(\omega_{m^{\prime}}^{2}+\lambda_{n^{\prime}}\right)}+(2) \frac{\tilde{G}_{3}^{I}\left(l, n, n^{\prime}\right) \tilde{G}_{3}^{I}\left(l^{\prime}, n, n^{\prime}\right)}{\left(\omega_{m}^{2}+\gamma_{n^{\prime}}\right) \omega_{m^{\prime}}^{2}}\right] \delta_{w+w^{\prime}}
$$




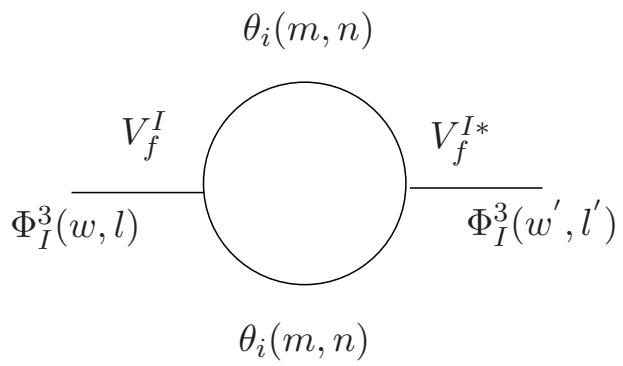

Figure 15. Feynman diagram with three-point vertex $V_{f}^{I}$ and its complex conjugate.

where $w=m^{\prime}+m$. The first term in (5.76) involving the three-point vertex $G_{3}^{I}\left(l, n, n^{\prime}\right)$ involves the fields $\Phi_{I}^{(1.2)}, I \neq 1$ and $C_{m, n}$ s. The corresponding Feynman diagram is shown in figure 14(a). Similarly the second term in (5.76) involving the three-point vertex $\tilde{G}_{3}^{I}\left(l, n, n^{\prime}\right)$ involves the fields $\Phi_{I}^{(1.2)}, I \neq 1$ and $\tilde{C}_{m, n}$ s. The relevant Feynman diagram is shown in figure 14(b). The Feynman diagram involving the three-point vertex with fermions is drawn in figure 15 .

The corresponding amplitude is

$$
\Sigma_{\Phi_{I}^{3}-\Phi_{I}^{3}}^{3}=N \sum_{m, n, n^{\prime}}\left[(8) \frac{G_{f}^{I}\left(l, n, n^{\prime}\right) G_{f}^{I *}\left(l^{\prime}, n, n^{\prime}\right)}{\left(i \omega_{m}-\sqrt{\lambda_{n}^{\prime}}\right)\left(i \omega_{m^{\prime}}-\sqrt{\lambda_{n^{\prime}}^{\prime}}\right)}\right] \delta_{w+w^{\prime}}
$$

where $w=m^{\prime}+m$. As in the case of $\Phi_{1}^{3}$, we can use the orthogonality relation for Hermite Polynomials to compute exactly, the various vertices given in section E.2 and constituting the mass-squared corrections (5.75), (5.76) and (5.77). We do these computations after setting the external momenta $l=l^{\prime}=w=w^{\prime}=0$.

$$
\begin{aligned}
G_{1}^{I}\left[0,0, n, n^{\prime}\right] & =\delta_{n, n^{\prime}} \\
G_{2}^{I}[0,0, n, n] & =\delta_{n, n^{\prime}} \\
\tilde{G}_{2}^{I}\left[0,0, n, n^{\prime}\right] & =\delta_{n, n^{\prime}} \\
G_{3}^{I}\left[0, n, n^{\prime}\right] & =0 \\
\tilde{G}_{3}^{I}\left[0, n, n^{\prime}\right] & =\sqrt{2 n-1} \delta_{n-1, n^{\prime}} \\
G_{f}^{I}\left[0, n, n^{\prime}\right] & =i \delta_{n, n^{\prime}} .
\end{aligned}
$$

We now proceed to establish the UV finiteness of finite temperature mass-squared corrections to $\Phi_{I}^{3}$. We first analyze the large $n$-behaviour of the various vertices for the one-loop mass-squared corrections (5.75), (5.76) and (5.77). The four-point vertices in the amplitudes constituting the two-point function (5.75) are associated with only one kind of propagator. In the large $n$ limit the four-point vertices computed in (5.78), (5.79) and (5.80) can be written as

$$
G_{1}^{I}(0,0, n, n)=G_{2}^{I}(0,0, n, n)=\tilde{G}_{2}^{I}(0,0, n, n)=1
$$

where we have used the Kronecker deltas to set $n=n^{\prime}$. Note that the vertex $G_{3}^{I}\left(0, n, n^{\prime}\right)$ is identically zero for all values of $n$ as shown in (5.81). Furthermore the fermionic vertex 
$G_{f}^{I}\left(0, n, n^{\prime}\right)$ (5.83) can be exactly computed for all $n$ and remains the same in the UV limit. The remaining three-point bosonic vertex $\tilde{G}_{3}^{I}\left(0, n, n^{\prime}\right)(5.82)$ in the UV limit becomes

$$
\tilde{G}_{3}^{I}\left(0, n, n^{\prime}\right) \sim \sqrt{2 n}\left[\delta_{n^{\prime}, n-1}\right] .
$$

This large $n$ behaviour of the vertices in turn gives rise to the following asymptotic forms of the two-point functions for the tree-level massless field $\Phi_{I}^{3}$.

$$
\begin{aligned}
& \Sigma_{\Phi_{I}^{3}-\Phi_{I}^{3}}^{1} \sim \frac{1}{2} N \sum_{m, n}\left[(6 \times 2) \frac{1}{\left(\omega_{m}^{2}+\gamma_{n}\right)}+(2) \frac{1}{\left(\omega_{m}^{2}+\lambda_{n}\right)}+(2) \frac{1}{\left(\omega_{m}^{2}\right)}\right] \\
& \Sigma_{\Phi_{I}^{3}-\Phi_{I}^{3}}^{2} \sim-\frac{1}{2} q N \sum_{m, n}\left[\frac{(2 n)}{\omega_{m}^{2}\left(\omega_{m}^{2}+\lambda_{n}\right)}\right] .
\end{aligned}
$$

Thus the total bosonic contribution in the limit $n \rightarrow \infty$ can be written as

$$
\Sigma_{\Phi_{I}^{3}-\Phi_{I}^{3}}^{1}+\Sigma_{\Phi_{I}^{3}-\Phi_{I}^{3}}^{2} \sim N \sum_{m, n}\left[(8) \frac{1}{\left(\omega_{m}^{2}+2 n q\right)}\right] \sim \sum_{n} \frac{4}{\sqrt{2 n}} .
$$

In this large $n$ limit, the contribution from the fermions coming from $\Sigma_{\Phi_{I}^{3}-\Phi_{I}^{3}}^{3}$ is same as the right hand side of eqn (5.88) with opposite sign.

Combining the vertices in (5.78)-(5.83) with their respective propagators (see appendix $(\mathrm{F})$ ), the effective mass-squared corrections for $\Phi_{I}^{3}, I \neq 1$, can be written down as a function of $q$ and $\beta$ in the following form;

$$
m_{\Phi_{I}^{3}}^{2}(q, \beta)=m_{I 0}^{2}+m_{I 1}^{2}(q, \beta),
$$

where $m_{I 0}^{2}$ and $m_{I 1}^{2}(q, \beta)$ denote the zero temperature quantum corrections and the finite temperature corrections respectively to the tree-level massless field $\Phi_{I}^{3}, I \neq 1$. The zero temperature quantum correction here is made dimensionless in the same way as in the case of $m_{10}^{2}$ in (5.71) and can be exactly computed as in the case of $\Phi_{1}^{3}$ and found to be

$$
m_{I 0}^{2}=\sum_{n=0}^{\infty} \frac{3}{\sqrt{2 n+1}}+\sum_{n=1}^{\infty} \frac{1}{\sqrt{2 n-1}}-\sum_{n=1}^{\infty} \frac{4}{\sqrt{2 n}}
$$

for all $n$, where the first two terms under summation come from the bosonic contributions and the last term comes from the fermionic contributions. The various sums in (5.90) can be reorganized and written in terms of the regularized Riemann Zeta function $\zeta\left(\frac{1}{2}\right)$. The dimensionless zero temperature quantum correction can be evaluated as

$$
m_{I 0}^{2}=\left(4(1-\sqrt{2}) \zeta\left(\frac{1}{2}\right)-1\right)=1.495
$$

Similarly the finite temperature part $m_{I 1}^{2}(q, \beta)$ can be written as,

$$
\begin{aligned}
m_{I 1}^{2}(q, \beta)= & \sum_{n=0}^{\infty} \frac{6}{\sqrt{2 n+1}} \frac{1}{e^{\sqrt{(2 n+1) q} \beta}-1}+\sum_{n=1}^{\infty} \frac{2}{\sqrt{2 n-1}} \frac{1}{e^{\sqrt{(2 n-1) q} \beta}-1} \\
& +\sum_{n=1}^{\infty} \frac{8}{\sqrt{2 n}} \frac{1}{e^{\sqrt{2 n q} \beta}+1} .
\end{aligned}
$$




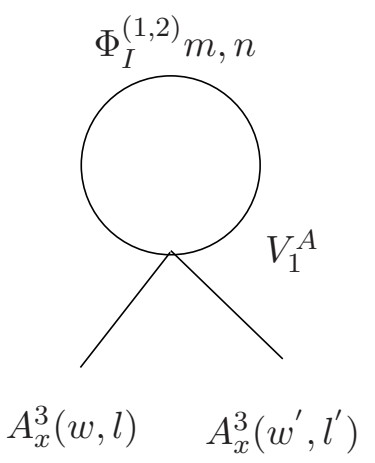

(a)

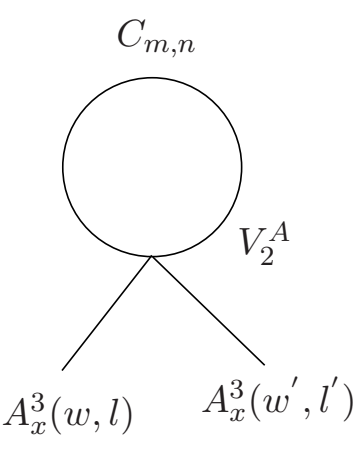

(b)

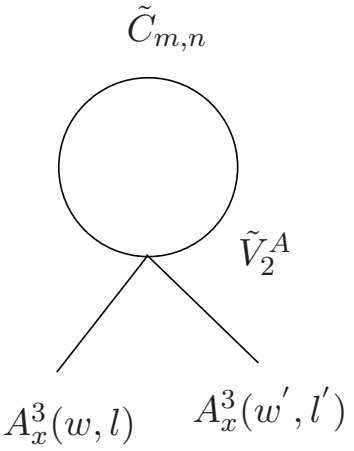

(c)

Figure 16. Feynman diagrams with four-point vertices $V_{1}^{A}, V_{2}^{A}, \tilde{V}_{2}^{A}$.

\subsection{Two point function for $A_{x}^{3}$}

We give below that expression for the two point one loop amplitude for $A_{x}^{3}$. The vertices are worked out in appendix E.3. The Feynman diagrams comprising the four-point bosonic interactions is given in figure 16 .

The amplitudes that are represented by the Feynman diagrams in figure 16 are collected together into the two-point finite temperature mass-squared corrections to the tree-level massless field $A_{x}^{3}$ in the following equation, namely

$$
\Sigma_{A_{x}^{3}-A_{x}^{3}}^{1}=\frac{1}{2} N \sum_{m, n}\left[(7 \times 2) \frac{G_{1}^{A}\left(n, n, l, l^{\prime}\right)}{\left(\omega_{m}^{2}+\gamma_{n}\right)}+(2) \frac{G_{2}^{A}\left(n, n, l, l^{\prime}\right)}{\left(\omega_{m}^{2}+\lambda_{n}\right)}+(2) \frac{\tilde{G}_{2}^{A}\left(n, n, l, l^{\prime}\right)}{\omega_{m}^{2}}\right] \delta_{w+w^{\prime}} .
$$

The first second and third terms in (5.93) are represented by the Feynman diagrams in figures 16(a), 16(b) and 16(c) respectively. The fields involved in four-point vertices in the first, second and third terms are $\Phi_{I}^{(1,2)}, I \neq 1, C_{m, n}$ and $\tilde{C}_{m, n}$ respectively. The Feynman diagrams depicting the various three-point bosonic interactions including $A_{x}^{3}$ are presented in figure 17 .

$$
\begin{aligned}
\Sigma_{A_{x}^{3}-A_{x}^{3}}^{2}= & -\frac{1}{2} q N \sum_{m, n, n^{\prime}}\left[\frac{G_{3}^{A}\left(n, n^{\prime}, l\right) G_{3}^{A}\left(n, n^{\prime}, l^{\prime}\right)}{\left(\omega_{m}^{2}+\lambda_{n}\right)\left(\omega_{m^{\prime}}^{2}+\lambda_{n^{\prime}}\right)}+\frac{\tilde{G}_{3}^{A}\left(n, n^{\prime}, l\right) \tilde{G}_{3}^{A}\left(n, n^{\prime}, l^{\prime}\right)}{\left(\omega_{m}^{2}\right)\left(\omega_{m^{\prime}}^{2}\right)}\right. \\
& \left.+(2) \frac{\tilde{G}_{3}^{A^{\prime}}\left(n, n^{\prime}, l\right) \tilde{G}_{3}^{A^{\prime}}\left(n, n^{\prime}, l^{\prime}\right)}{\left(\omega_{m}^{2}+\lambda_{n}\right)\left(\omega_{m^{\prime}}^{2}\right)}+(7) \frac{G_{4}^{A}\left(n, n^{\prime}, l\right) G_{4}^{A}\left(n, n^{\prime}, l^{\prime}\right)}{\left(\omega_{m}^{2}+\gamma_{n}\right)\left(\omega_{m^{\prime}}^{2}+\gamma_{n^{\prime}}\right)}\right] \delta_{w+w^{\prime}} .
\end{aligned}
$$

where $w=m^{\prime}+m$. The first term in (5.94) comprising the three-point vertex $G_{3}^{A}\left(l, n, n^{\prime}\right)$ involves the fields $C_{m, n}$ and $C_{m, n}^{\prime}$. in the loops and is represented by the Feynman diagram in figure $17(\mathrm{a})$. The second term in (5.94) involving the vertex $\tilde{G}_{3}^{A}\left(l, n, n^{\prime}\right)$ comprises of the fields $\tilde{C}_{m, n} \mathrm{~s}$ and $\tilde{C}_{m, n}^{\prime}$ in the loop. The corresponding Feynman diagram is given in figure $17(\mathrm{~b})$. The third term has contributions from $\tilde{C}_{m, n} \mathrm{~S}$ and $\tilde{C}_{m, n}$ in the loop with Feynman diagram shown in figure 17(c). Lastly the fourth term depicted in figure 17(d) has contributions from the fields $\Phi_{I}^{(1,2)}, I \neq 1$. 


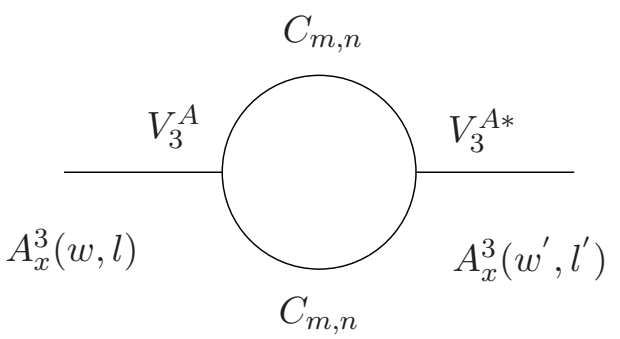

(a)

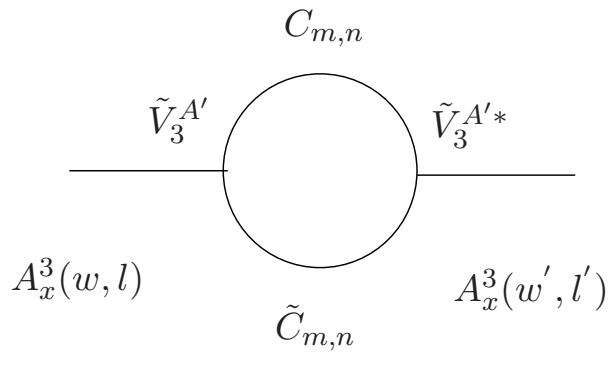

(c)

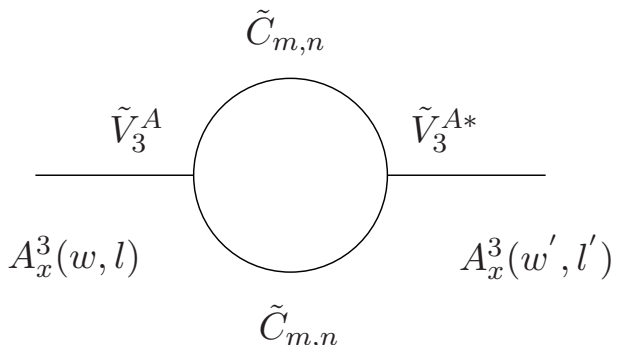

(b)

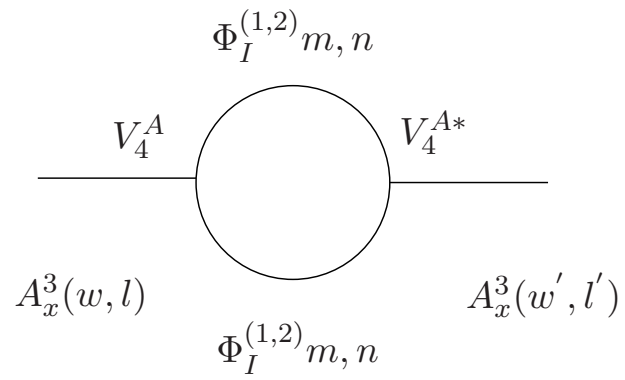

(d)

Figure 17. Feynman diagrams with three-point vertices $V_{3}^{A}, \tilde{V}_{3}^{A}, \tilde{V}_{3}^{A^{\prime}}$ and $V_{3}^{4}$.

Similarly the amplitude involving fermions in the loop is (5.95). The corresponding Feynman diagrams are presented in figure 18.

$$
\begin{aligned}
\Sigma_{A_{x}^{3}-A_{x}^{3}=\frac{1}{2} q N \sum_{m, n, n^{\prime}}}\left[\begin{array}{l}
(16) \\
\left(i \omega_{m}-\sqrt{\lambda_{n}^{\prime}}\right)\left(i \omega_{m^{\prime}}^{A 1}-\sqrt{\lambda_{n^{\prime}}^{\prime}}\right)
\end{array}\right. \\
\left.\left.\quad-(16) \frac{G_{f}^{A 2}\left(n, n^{\prime}, l\right) G_{f}^{A 2 *}\left(n, n^{\prime}, l^{\prime}\right)}{\left(i \omega_{m}-\sqrt{\lambda_{n}^{\prime}}\right)\left(-i \omega_{m^{\prime}}-\sqrt{\lambda_{n^{\prime}}^{\prime}}\right)}\right)\right] \delta_{w+w^{\prime}}^{A 1 *} .
\end{aligned}
$$

where $w=m^{\prime}+m$. The fermionic three-point vertex $G_{f}^{A 1}\left(n, n^{\prime}, l\right)$ constituting the first term in the two-point function (5.95) has contributions from the fermionic fields $\theta_{i}(m, n)$. The amplitude is represented in the Feynman diagram presented in figure 18(a). The second term in (5.95) on the other hand involves the fields $\theta_{i}(m, n)$ and their complex conjugate $\theta_{i}^{*}(m, n)$ in the vertex $G_{f}^{A 2}\left(n, n^{\prime}, l\right)$. The corresponding Feynman diagram is given in figure $18(\mathrm{~b})$.

The vertices given in section E.3 and participating in the two-point functions that produce the mass-squared corrections to the tree-level massless field $A_{x}^{3}$ can be exactly computed following the same procedure as discussed for $\Phi_{1}^{3}$ and $\Phi_{I}^{3}, I \neq 1$.

$$
G_{1}^{A}\left[n, n^{\prime}, 0,0\right]=\delta_{n, n^{\prime}}, \quad G_{2}^{A}\left[n, n^{\prime}, 0,0\right]=\frac{1}{2} \delta_{n, n^{\prime}}, \quad \tilde{G}_{2}^{A}\left[n, n^{\prime}, 0,0\right]=\frac{1}{2} \delta_{n, n^{\prime}}
$$




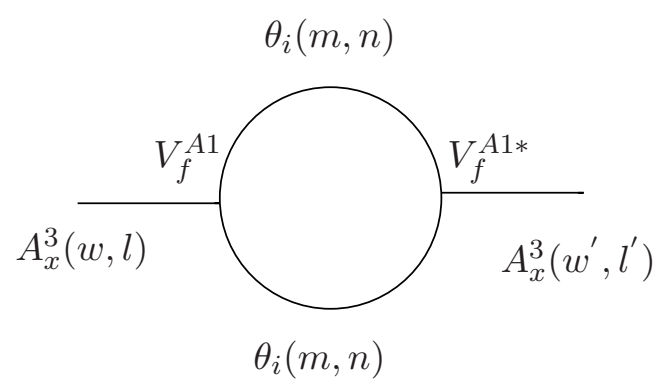

(a)

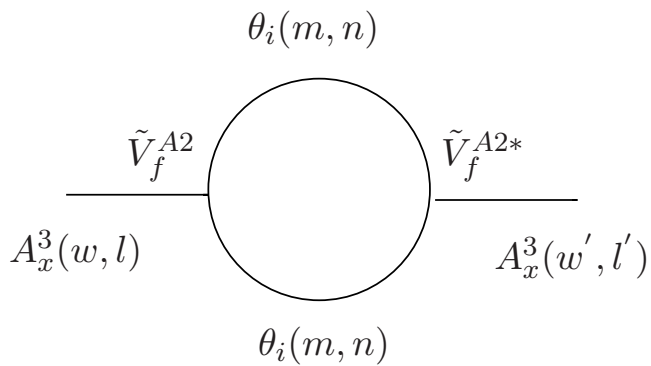

(b)

Figure 18. Feynman diagrams involving three-point vertices $V_{f}^{A 1}, V_{f}^{A 2}$.

$$
\begin{aligned}
G_{3}^{A}\left[n, n^{\prime}, 0\right] & =2\left(\sqrt{\frac{2 n(n+1)(n-1)}{(2 n-1)(2 n+1)}} \delta_{n+1, n^{\prime}}-\sqrt{\frac{2 n(n-1)(n-2)}{(2 n-1)(2 n-3)}} \delta_{n-1, n^{\prime}}\right), \\
\tilde{G}_{3}^{A}\left[n, n^{\prime}, 0\right] & =0 \\
\tilde{G}_{3}^{A^{\prime}}\left[n, n^{\prime}, 0\right] & =-\sqrt{2}\left(\frac{(n+1) \sqrt{n-1}}{\sqrt{(2 n-1)(2 n+1)}} \delta_{n+1, n^{\prime}}+\frac{\sqrt{n}(n-1)}{\sqrt{(2 n-1)(2 n-3)}} \delta_{n-1, n^{\prime}}\right), \\
G_{4}^{A}\left[n, n^{\prime}, 0\right] & =\left(\sqrt{2(n+1)} \delta_{n+1, n^{\prime}}-\sqrt{2 n} \delta_{n-1, n^{\prime}}\right) \\
G_{f}^{A 1}\left[n, n^{\prime}, 0\right] & =-\frac{i}{2}\left(\delta_{n+1, n},+\delta_{n-1, n^{\prime}}\right) \\
G_{f}^{A 2}\left[n, n^{\prime}, 0\right] & =-\frac{i}{2}\left(2 \delta_{n+1, n},-2 \delta_{n-1, n^{\prime}}\right) .
\end{aligned}
$$

In the same spirit as for $\Phi_{1}^{3}$ and $\Phi_{I}^{3}$ we now proceed to establish the UV finiteness for the one-loop two-point functions for the field $A_{x}^{3}$. In the large $n$ limit the various vertices in eqns (5.96)-(5.102) reduce to

$$
\begin{array}{llrl}
G_{1}^{A}(n, n, 0) & =1 ; & G_{2}^{A}(n, n, 0) & =\tilde{G}_{2}^{A}(n, n, 0,0)=\frac{1}{2} \\
G_{3}^{A}\left(n, n^{\prime}, 0\right) & \sim \frac{\sqrt{2 n}}{8}\left[8 \delta_{n^{\prime}, n-1}-8 \delta_{n^{\prime}, n+1}\right] ; & \tilde{G}_{3}^{A^{\prime}}\left(n, n^{\prime}, 0\right) \sim-\frac{\sqrt{2 n}}{8}\left[4 \delta_{n^{\prime}, n-1}+4 \delta_{n^{\prime}, n+1}\right] \\
\tilde{G}_{3}^{A}\left(n, n^{\prime}, 0\right)=0 ; & \tilde{G}_{4}^{A}\left(n, n^{\prime}, 0\right) \sim \sqrt{2 n}\left[\delta_{n^{\prime}, n+1}-\delta_{n^{\prime}, n-1}\right] .
\end{array}
$$

With these, the amplitudes in the ultraviolet limit is same as the right hand side of the equations (5.66), (5.66) and (5.70), thus showing that the one-loop $A_{x}^{3}-A_{x}^{3}$ amplitude is ultraviolet finite.

Once again we write down the effective mass-squared for the field $A_{x}^{3}$ as a function of $q$ and $\beta$ as

$$
m_{A_{x}^{3}}^{2}(q, \beta)=m_{x 0}^{2}+m_{x 1}^{2}(q, \beta)
$$

where $m_{x 0}^{2}$ denotes the dimensionless (same as $m_{10}^{2}$ and $m^{2} I 0$ ) zero temperature quantum corrections and $m_{x 1}^{2}(q, \beta)$ denotes the temperature dependent mass-squared corrections 
for the tree-level massless field $A_{x}^{3}$. The zero temperature quantum corrections can be written as

$$
\begin{aligned}
m_{x 0}^{2}= & {\left[\sum_{n=0}^{\infty} \frac{7}{2 \sqrt{2 n+1}}+\sum_{n=2}^{\infty} \frac{1}{4 \sqrt{2 n-1}}-\frac{7}{4} \sum_{n=0}^{\infty}\left(\frac{1}{\sqrt{2 n+1}}-\frac{n+1}{\sqrt{2 n+3}}\right)-\frac{7}{4} \sum_{n=1}^{\infty} \frac{n}{\sqrt{2 n-1}}\right.} \\
& -\sum_{n=2}^{\infty}\left(\frac{n(n-1)}{(2 n+1)(2 n-3)} \frac{1}{\sqrt{2 n-1}}-\frac{n(n+1)(n-1)}{(2 n-1)(2 n+1)} \frac{1}{\sqrt{2 n+1}}+\frac{n(n-1)(n-2)}{(2 n-1)(2 n-3)} \frac{1}{\sqrt{2 n-3}}\right) \\
& \left.+\sum_{n=2}^{\infty}\left(\frac{(n-1)(n+1)^{2}}{(2 n-1)^{\frac{5}{2}}(2 n+1)}+\frac{n(n-1)^{2}}{(2 n-1)^{\frac{5}{2}}(2 n-3)}\right)\right] \\
& -\sum_{n=1}^{\infty} \frac{2}{\sqrt{2 n}+\sqrt{2(n+1)}}+\frac{2}{\sqrt{2 n}+\sqrt{2(n-1)}} .
\end{aligned}
$$

We compute the dimensionless zero temperature quantum corrections given by (5.107) numerically. The convergent value is,

$$
m_{x 0}^{2}=1.514
$$

The temperature dependent part in (5.106) can be written as

$$
\begin{aligned}
& m_{x 1}^{2}(q, \beta)=\left[\sum_{n=0}^{\infty} \frac{7}{\sqrt{2 n+1}} \frac{1}{\left(e^{\sqrt{(2 n+1) q} \beta}-1\right)}+\sum_{n=2}^{\infty} \frac{1}{2 \sqrt{2 n-1}} \frac{1}{\left(e^{\sqrt{(2 n-1) q} \beta}-1\right)}\right. \\
& -\frac{7}{2} \sum_{n=1}^{\infty}\left(\frac{1}{\sqrt{2 n+1}} \frac{1}{\left(e^{\sqrt{(2 n+1) q} \beta}-1\right)}-\frac{n+1}{\sqrt{2 n+3}} \frac{1}{\left(e^{\sqrt{(2 n+3) q} \beta}-1\right)}\right) \\
& -\frac{7}{2} \sum_{n=1}^{\infty} \frac{n}{\sqrt{2 n-1}} \frac{1}{\left(e^{\sqrt{(2 n-1) q} \beta}-1\right)}-2 \sum_{n=2}^{\infty}\left(\frac{n(n-1)}{(2 n+1)(2 n-3)} \frac{1}{\sqrt{2 n-1}} \frac{1}{\left(e^{\sqrt{(2 n-1) q} \beta}-1\right)}\right. \\
& \left.-\frac{n(n+1)(n-1)}{(2 n-1)(2 n+1)^{\frac{3}{2}}} \frac{1}{\left(e^{\sqrt{(2 n+1) q} \beta}-1\right)}+\frac{n(n-1)(n-2)}{(2 n-1)(2 n-3)^{\frac{3}{2}}} \frac{1}{\left(e^{\sqrt{(2 n-3) q} \beta}-1\right)}\right) \\
& +2 \sum_{n=2}^{\infty}\left(\frac{(n-1)(n+1)^{2}}{(2 n-1)^{\frac{5}{2}}(2 n+1)}+\frac{n(n-1)^{2}}{(2 n-1)^{\frac{5}{2}}(2 n-3)}\right) \frac{1}{\left(e^{\sqrt{(2 n-1) q} \beta}-1\right)} \\
& \left.+\left(\frac{1}{2}-2 \sum_{n=2}^{\infty}\left(\frac{(n-1)(n+1)^{2}}{(2 n-1)(2 n+1)^{2}}+\frac{n(n-1)^{2}}{(2 n-1)(2 n-3)^{2}}\right)\right) \sum_{m=-\infty}^{\infty} \frac{\sqrt{q} \beta}{4 \pi^{2} m^{2}}\right] \\
& +\left[\sum _ { n = 1 } ^ { \infty } \left(\left(2 \frac{(2 \sqrt{2 n}+\sqrt{2(n-1)}+\sqrt{2(n+1)})}{(\sqrt{2 n}+\sqrt{2(n-1)})(\sqrt{2 n}+\sqrt{2(n+1)})}\right.\right.\right. \\
& \left.+8 \frac{(\sqrt{2(n+1)}+\sqrt{2(n-1)}-2 \sqrt{2 n})}{(\sqrt{2 n}-\sqrt{2(n-1)})(\sqrt{2(n+1)}-\sqrt{2 n})}\right) \frac{1}{\left(e^{\sqrt{2 n q} \beta}+1\right)} \\
& +\left(\frac{2}{\sqrt{2(n-1)}+\sqrt{2 n}}-\frac{8}{\sqrt{2 n}-\sqrt{2(n-1)}}\right) \frac{1}{\left(e^{\sqrt{2(n-1) q} \beta}+1\right)} \\
& \left.\left.+\left(\frac{2}{\sqrt{2(n+1)}+\sqrt{2 n}}+\frac{8}{\sqrt{2(n+1)}-\sqrt{2 n}}\right) \frac{1}{\left(e^{\sqrt{2(n+1)} \beta}+1\right)}\right)\right] \text {. }
\end{aligned}
$$


In the end the effective masses-squared for the massless fields namely $m_{\Phi_{1}^{3}}^{2}, m_{\Phi_{I}^{3}}^{2}$ and $m_{A_{x}^{3}}^{2}$ depend only on the parameter $q$ and temperature. Later in section 6.1, we present the behaviour of the effective masses-squared with temperature for different values of $q$.

\section{$6 \quad$ Finite part of effective tachyon mass}

Having computed the temperature corrected one-loop mass-squared for the various massless fields, we can now proceed to compute the mass-squared corrections for the tree-level tachyons. We have already established the UV finiteness of the tachyonic amplitudes by demonstrating the cancellation of leading order divergences from the zero temperature bosonic and fermionic quantum corrections to the tree-level tachyon mass-squared. However given the fairly complicated mathematical form of the various corrections given in (4.5), (4.6) and (4.7), extracting the finite part of the amplitudes appears to be very difficult. Hence we are unable to give analytical expressions for the finite part of the zero temperature quantum corrections to the tree-level tachyon mass-squared. This complications also prevents us from computing the transition temperature analytically. Given these handicaps we are compelled to resort to numerical means. In the following section we present a numerical computation of the transition temperature.

\subsection{Numerical results}

The computation of the one-loop finite temperature mass-squared for all the tree-level massless degrees of freedom is crucial because these temperature dependent masses modify the corresponding propagators of the massless fields thereby ensuring infra-red finiteness of the one-loop effective masses-squared of the tachyons. All the finite temperature corrections to the tree-level masses-squared are now shown to be UV finite. The tachyonic instability in the bulk is proposed to give rise to BCS Cooper-pairing instability in the boundary theory [1]. In this section we demonstrate that the instability is removed by finite temperature effects. The tree-level tachyon mass-squared is $-\frac{q}{g^{2}}$, where $g$ is the dimensionfull Yang-Mills coupling in $(1+1)$-dimensions. The finite temperature one-loop correction including the zero temperature quantum corrections is $\mathcal{O}(1)$. The temperature-dependent mass-squared corrections is always increasing and there exists a critical temperature where the effective mass-squared of the tachyonic fields become zero. Beyond the critical temperature the effective mass-squared of the tachyon is found to be positive and increasing. This bears hallmark of a phase-transition from the unstable phase to the stable phase. In the boundary theory this is proposed in [1] to correspond to a superconducting phase-transition. As mentioned in sections 1 and 6 , the critical temperature of phase transition cannot be computed analytically. We therefore tread a different path. We demonstrate numerically the behaviour of the masses-squared with varying $\beta$ as well as $T$ due to zero temperature quantum corrections + the finite temperature effects without computing them separately. In all the mass-squared corrections, the UV divergent pieces in the zero temperature corrections from the bosonic side cancel with that from the fermionic side. At large values of the momenta $n$ and $l$ the finite part of the quantum corrections fall off very sharply and eventually only the finite temperature corrections dominate. The parameter $q$ provides a 
scale for supersymmetry breaking in the present brane-configuration under study and has the dimension of mass-squared. It is also related to the angle between the branes as

$$
q=\frac{1}{\pi \alpha^{\prime}} \tan \left(\frac{\theta}{2}\right)
$$

The two-point functions for the tachyons have infra-red problem due to the presence of massless fields in the loops. As mentioned in section 5.2 we need to modify the propagators of the tree-level massless fields by introducing a mass-squared shift provided by the finite temperature corrections to their tree-level masses-squared. The leading order behaviour of the temperature dependent part of the mass-squared corrections in $1+1$-dimensions is linear with increasing temperature at high temperatures. The finite temperature effective mass-squared of the tree-level tachyon in $(1+1)$-dimensions can thus be estimated within perturbation theory to be (in dimensionless variables)

$$
m_{\mathrm{eff}}^{2}\left(C_{0,0}\right)=-\frac{q}{g^{2}}+[m_{0}^{2}+\frac{T}{\sqrt{q}} \underbrace{\left(\sum_{n} \frac{1}{\lambda_{n}}+\cdots\right)}_{=x}]+\mathcal{O}\left(\frac{g^{2}}{q}\right) .
$$

The physical mass is thus $m_{\text {eff }}^{2} g^{2}$ and $m_{0}^{2}$ represents the dimensionless zero temperature quantum corrections independent of $q$ and $g^{2}$ and collected from all the amplitudes in equations (4.7), (4.8) and (4.36). In equation (6.2), $x$ is a dimensionless number (independent of $g^{2}$, and $q$ ) specifying the temperature dependent contribution. In equation (6.2), $\mathcal{O}\left(\frac{g^{2}}{q}\right)$ represents the next higher order in quantum corrections given by two-loop Feynman diagrams. The dimensionless zero temperature quantum correction to the tree-level tachyon mass-squared and is found numerically to be approximately equal to $m_{0}^{2}=1.6$. The behaviour of the finite temperature masses-squared of the massless fields as well as the tachyonic fields are depicted pictorially by plotting the masses-squared against temperature $T$ and $\beta=\frac{1}{T}$. We proceed to present the plots.

In all the plots we have displayed the one-loop effective masses-squared as multiplied by $g^{2}$. The figures 19, 21 and 23 depict the behaviour of the masses-squared namely $m_{\phi_{1}^{3}}^{2}$, $m_{\phi_{I}^{3}}^{2}$ and $m_{A_{x}^{3}}^{2}$ with varying $\beta$. The mass-squared decreases with increasing $\beta$ as expected. In the figures 20, 22 and $24, m_{\phi_{1}^{3}}^{2}, m_{\phi_{I}^{3}}^{2}$ and $m_{A_{x}^{3}}^{2}$ are shown to increase almost linearly with increasing temperature. This behaviour is expected from finite temperature field theory as finite temperature corrections are always known to be positive and increasing.

As discussed in section 5.3, we also calculate the mass-matrix for the massless modes $\tilde{C}_{w, k}$ numerically and diagonalize the matrix. Using the temperature dependent massessquared of the various massless fields discussed above the effective mass-squared for the fields $C_{0,0}$ can be evaluated numerically as a function of $\beta$ (or $T$ ). The effective masssquared for the tree-level tachyon are plotted against $\beta$ in the figure 25 . for three values of $q$, namely $q=0.1$ and $q=0.2$ and $q=0.3$ and $g^{2}=0.01$. The plots of the masssquared against temperature $T$ for $q=0.1$ and $q=0.2$ and $q=0.3$ are given in figure 26 and figure 27. As expected the finite temperature corrections dominate with increasing 


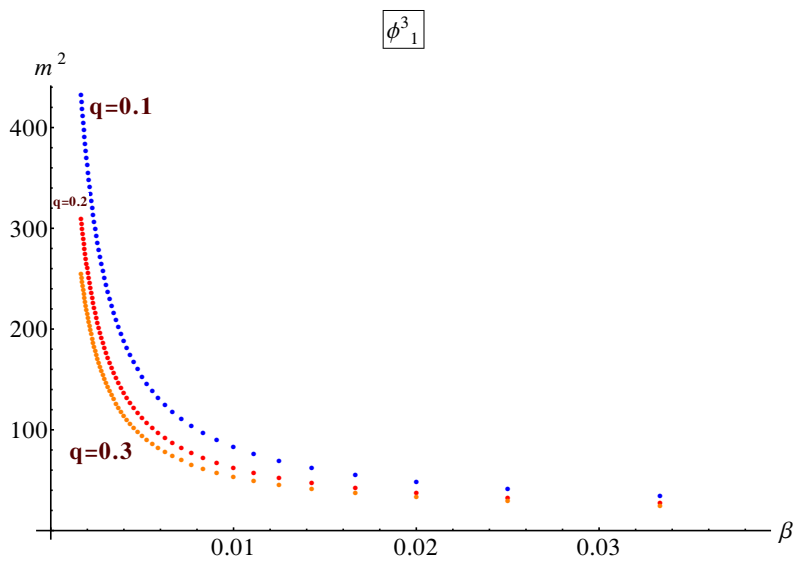

Figure 19. Plots of the mass-squared correction to the massless field $\phi_{1}^{3}$ against $\beta=\frac{1}{T}$ for $g^{2}=0.01$ and $q=0.1,0.2,0.3$.

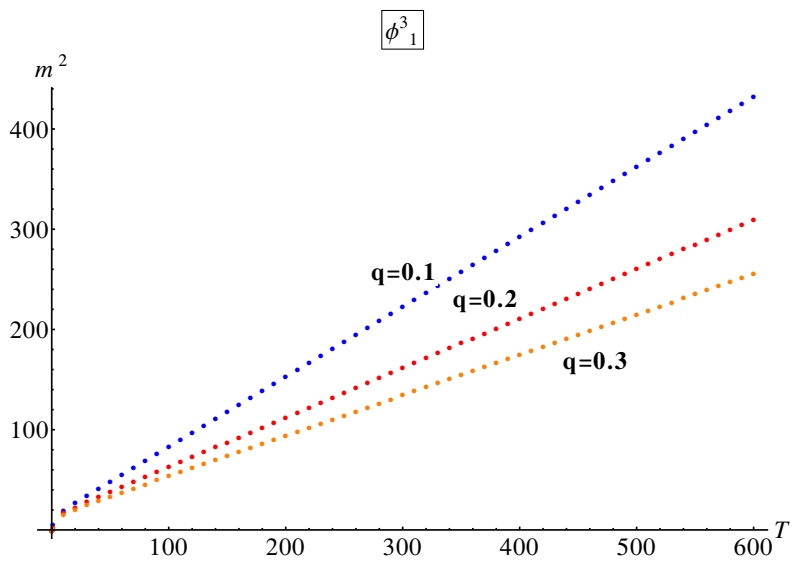

Figure 20. Plot of the mass-squared correction to the massless field $\phi_{1}^{3}$ against $T$ for $g^{2}=0.01$ and $q=0.1,0.2,0.3$.

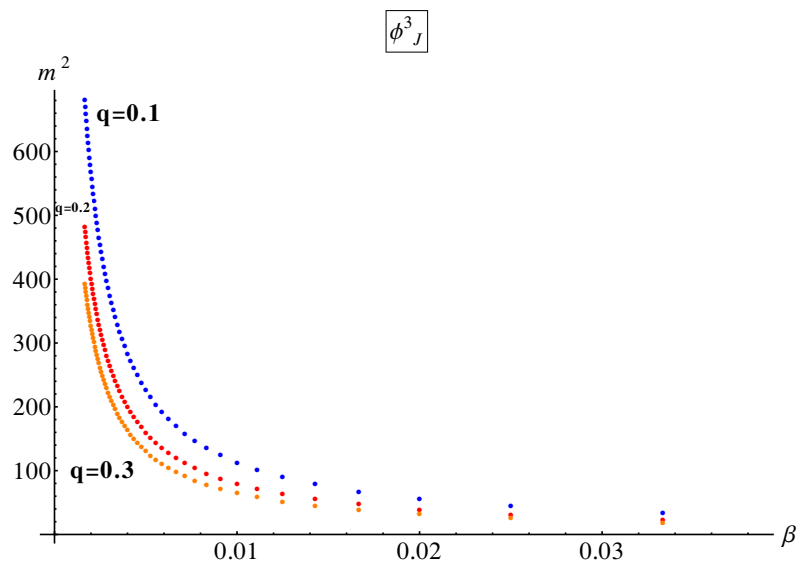

Figure 21. Plot of the mass-squared correction to the massless field $\phi_{I}^{3}$ against $\beta=\frac{1}{T}$ for $g^{2}=0.01$ and $q=0.1,0.2,0.3$. 


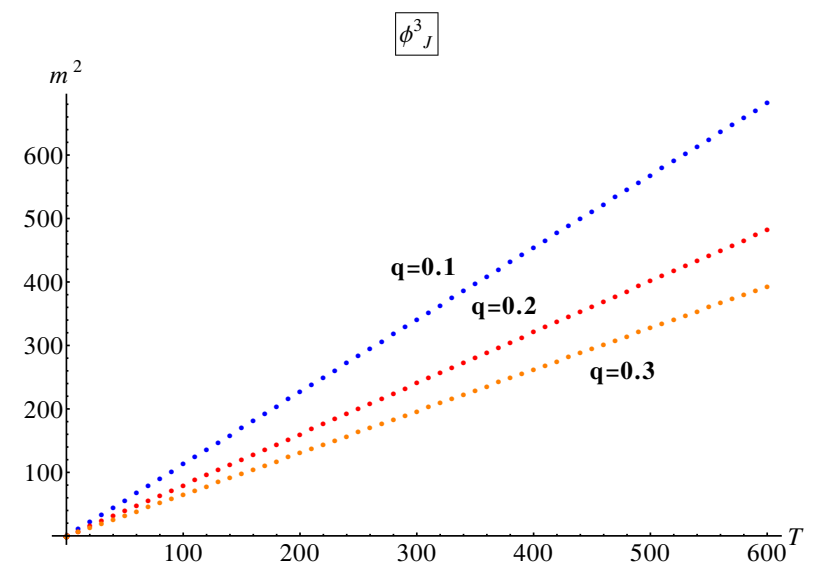

Figure 22. Plot of the mass-squared correction to the massless field $\phi_{I}^{3}$ against $T$ for $g^{2}=0.01$ and $q=0.1,0.2,0.3$.

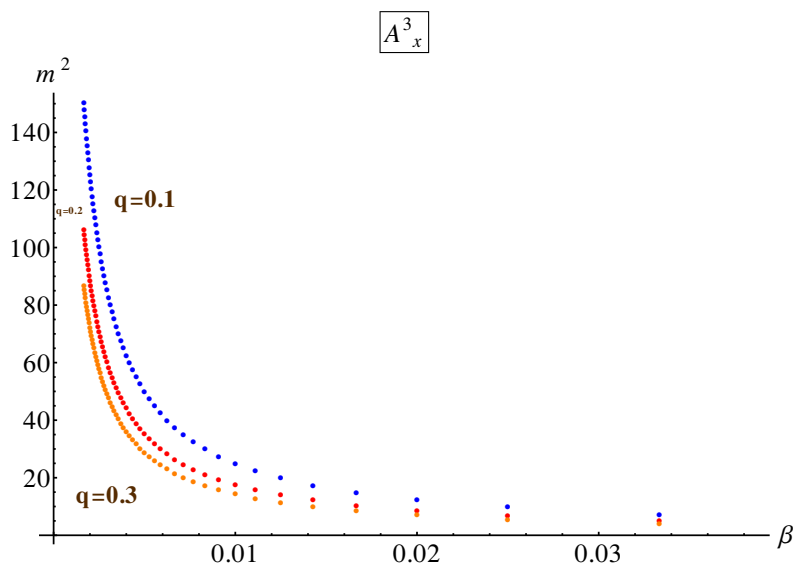

Figure 23. Plot of the mass-squared correction to the massless field $A_{x}^{3}$ against $\beta=\frac{1}{T}$ for $g^{2}=0.01$ and $q=0.1,0.2,0.3$.

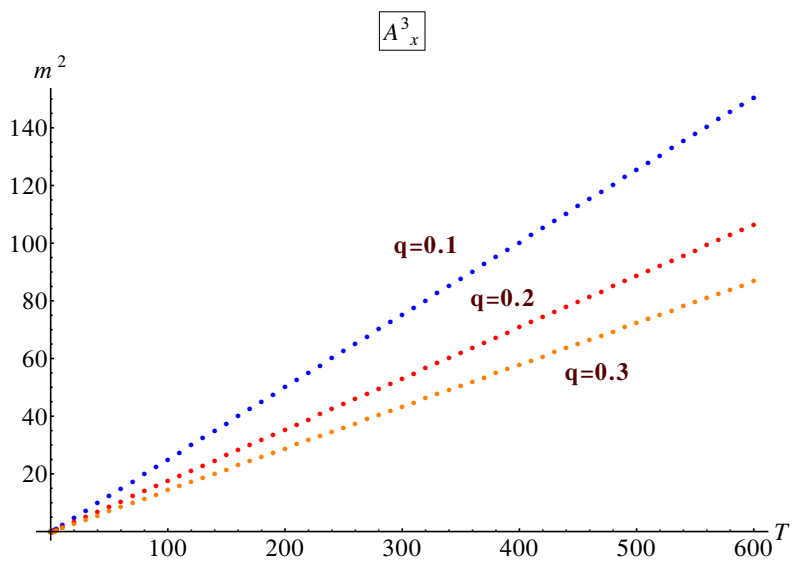

Figure 24. Plot of the mass-squared correction to the massless field $A_{x}^{3}$ against $T$ for $g^{2}=0.01$ and $q=0.1,0.2,0.3$. 


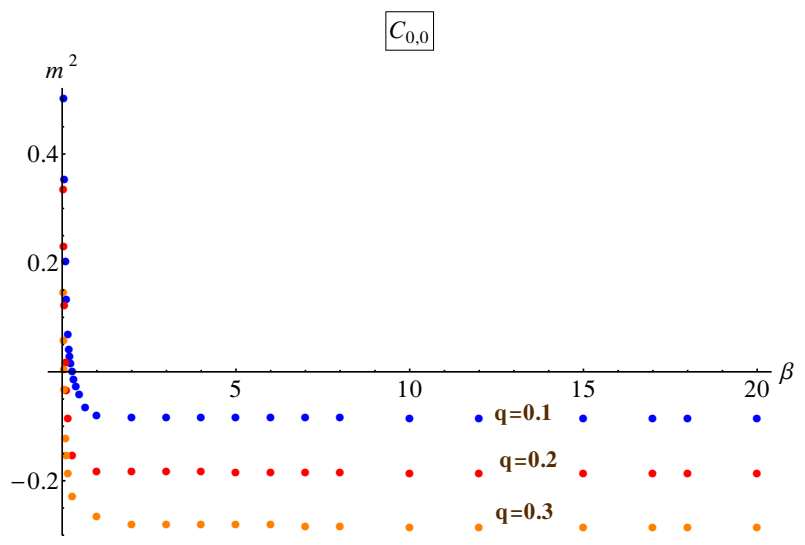

Figure 25. Plot of the mass-squared correction to the tree-level tachyon against $\beta=\frac{1}{T}$ for $g^{2}=0.01, q=0.1,0.2$ and 0.3 .

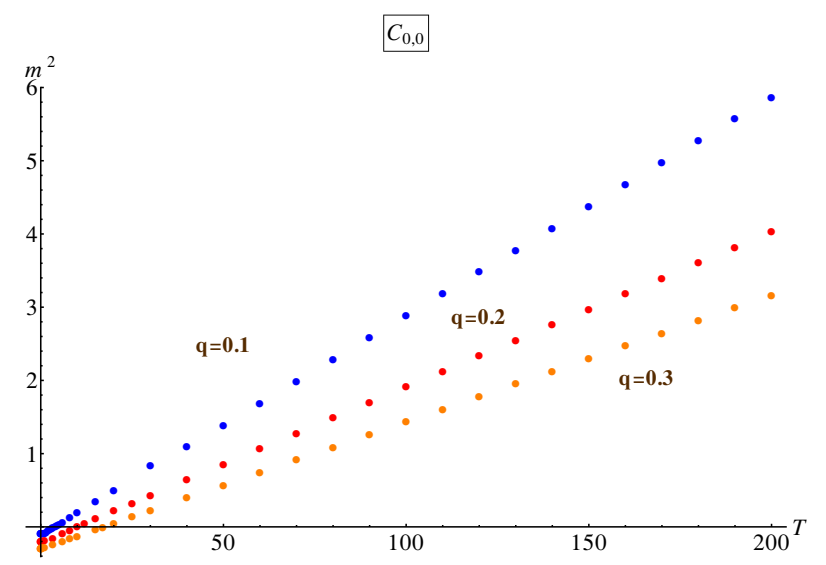

Figure 26. Plot of the mass-squared correction to the tree-level tachyons against $T$ for $g^{2}=0.01$, $q=0.1,0.2$ and 0.3 .

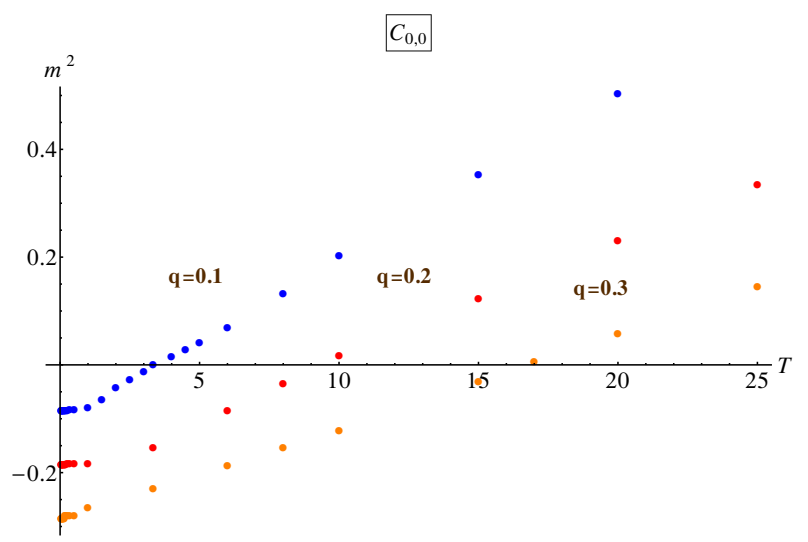

Figure 27. Plot of the mass-squared correction to the tree-level tachyons against $T$ for $g^{2}=0.01$, $q=0.1,0.2$ and 0.3 . 
temperature which is clear from the $m_{\text {eff }}^{2}\left(C_{0,0}\right)$ vs $T$ plots in figure 26 where the behaviour of the mass-squared appears to be almost linear at higher temperatures. The effective masssquared $m_{\text {eff }}^{2}\left(C_{0,0}\right)$ is equal to zero at a value of temperature (the transition temperature $T_{c}$ ) where the graphs intersect the $\beta$ and $T$-axes in the plots given in figure 25 , figure 26 and figure 27. The plots in figure 27 are drawn for smaller range of $T$ in order to show the critical points $\left(m_{\text {eff }}^{2}\left(C_{0,0}\right)=0\right)$ more clearly. Putting $m_{\text {eff }}^{2}\left(C_{0,0}\right)=0$ in equation (6.2) we get

$$
T_{c}=\frac{1}{x}\left[q^{\frac{1}{2}}\left(\frac{q}{g^{2}}-m_{0}^{2}\right)\right]
$$

where $T_{c}$ is the dimensionfull transition temperature (having dimension of mass). One can also define a dimensionless transition temperature by $T_{c}=\tilde{T}_{c} q^{\frac{3}{2}} / g^{2}$. One should note that the dimensionless tree-level mass-squared of the tachyon for $q=0.1,0.2$ and 0.3 are $q / g^{2}=10,20$ and 30 respectively for $g^{2}=0.01$, whereas the quantum correction $m_{0}^{2}$ is much smaller. Now if $m_{0}^{2}=0, \tilde{T}_{c}=\frac{1}{x}$ and is thus independent of $q, g^{2}$. Thus to a good approximation one expects that $\tilde{T}_{c}$ will be independent of $q, g^{2}$. One has to note that this scaling relation for the transition temperature is the dominant term at the level of one-loop. At the level of higher loops the dependence of the effective mass-squared on temperature will be much more complicated. However at least in weak coupling, the quantum corrections at successive higher loops will be smaller and smaller, and the dominant term in $\tilde{T}_{c}$ will still be given by this one loop relation. From the plots given in figure 25 and figure 27 , the numerical values of $T_{c}$ for $g^{2}=0.01$ and $q=0.1, q=0.2$ and $q=0.3$ are $T_{c}=3.34$, $T_{c}=9.48$ and $T_{c}=16.73$ respectively. This gives $\tilde{T}_{c}=1.0562,1.0599$ and 1.0182 for $q=0.1,0.2$ and 0.3 and $g^{2}=0.01$ respectively, confirming our expectation that when $m_{0}^{2}$ is small $\tilde{T}_{c}$ is approximately independent of $g, q$.

\section{Discussion and outlook}

We have computed the one-loop finite temperature corrections to the tree-level tachyon mass-squared in intersecting D1-branes in a self consistent manner. We have shown the UV finiteness (at one loop). We have seen that at high temperatures the tachyonic field becomes massive as expected and we have computed this critical temperature in a one loop approximation - improved by incorporating mass-squared corrections to the massless fields in the spirit of the RG. This takes care of the IR divergences. Thus our calculation has no UV or IR divergences.

This model resembles the holographic BCS superconductor model discussed in [1]. We expect that with the techniques developed in this paper it should be possible to tackle the model in [1] involving higher branes. These techniques should also be useful in other contexts where D brane constructions are used and supersymmetry spontaneously broken.

The entire computation is done in temporal gauge, $A_{0}^{a}=0$. This gauge choice helps to avoid ghosts in the theory. The original theory describing the world-volume of two D1branes is a (1+1)-dimensional supersymmetric SU(2) Yang-Mills theory which is a UV finite theory. The choice of the background $\left\langle\phi_{B}^{3}\right\rangle=q x$ breaks supersymmetry without tampering with the other degrees of freedom. Hence we find that the UV behaviour of the amplitudes 
in a broken supersymmetry scenario remains the same as in the supersymmetric case. In order to establish the UV finiteness of the one-loop corrections we rely on asymptotic expansion of the vertices and find that the leading order divergent pieces from the bosonic and fermionic loops cancel among themselves. The effective mass-squared of the tachyon is found to grow linearly with temperature in accordance to the expected behaviour in $(1+1)$ dimensions. The kinetic terms for the bosons are scale independent in $(1+1)$-dimensions. Hence the zero-temperature one loop quantum corrections are found to be independent of the supersymmetry breaking scale. The crossing of the $m_{\text {eff }}^{2}\left(C_{0,0}\right)$ vs $T$ curves from negative to positive values indicates two distinct phases. This bears the signature of a phase transition. We also find that the dimensionless critical temperature $\left(T_{c} g^{2} / q^{3 / 2}\right)$ for this phase transition has very closely placed values namely $\tilde{T}_{c}=1.0562,1.0599$ and 1.0182. In order to do the complete stability analysis of the intersecting $D 1$-branes at finite temperature one has to compute the full tachyon effective action at finite temperature which rely on higher loop calculations. The results demonstrated in this paper can be generalized to higher dimensional branes without much difficulty. In particular for two intersecting $D p$-branes the effective mass-squared for the tree-level tachyons is expected to grow as $T^{p-1}$.

\section{Acknowledgments}

The authors would like to acknowledge the Institute of Mathematical Sciences, Chennai for providing a free and vibrant academic atmosphere and the Department of Atomic Energy for providing funds for this project. S.P.C. would like to thank colleagues and compatriots Swastik Bhattacharya and Shankhadeep Chakrabortty, Saurabh Gupta and Akhilesh Nautiyal and T Geetha for fruitful as well as refreshing lighthearted discussions. S.S. would like to thank Institute of Mathematical Sciences for the kind hospitality during various stages of this work. The work of S.S. is partially supported by the Research and Development Grant (2013-2014), University of Delhi.

\section{A Dimensional reduction of $D=10, \mathcal{N}=1, \mathrm{SU}(2) \mathrm{SYM}$ to $D=2$}

We first write down the action for $D=10, \mathcal{N}=1 \mathrm{SYM},{ }^{2}$

$$
\begin{aligned}
S_{9+1} & =\frac{1}{g^{2}} \operatorname{tr} \int d^{10} x\left[-\frac{1}{2} F_{M N} F^{M N}+i \bar{\Psi} \Gamma^{M} D_{M} \Psi\right] \\
F_{M N} & =\partial_{M} A_{N}-\partial_{N} A_{M}-i\left[A_{M}, A_{N}\right] \\
D_{M} \Psi & =\partial_{M} \Psi-i\left[A_{M}, \Psi\right]
\end{aligned}
$$

where $M, N=0, \cdots 9$ with $A_{M}=\frac{\sigma^{a}}{2} A_{M}^{a}, \Psi=\frac{\sigma^{a}}{2} \Psi_{M}^{a}$ and,

$$
\left[\frac{\sigma^{a}}{2}, \frac{\sigma^{b}}{2}\right]=i \epsilon^{a b c} \frac{\sigma^{c}}{2} ; \quad \frac{1}{2} \operatorname{tr}\left(\sigma^{a} \sigma^{b}\right)=\delta^{a b}
$$

\footnotetext{
${ }^{2}$ We will use the metric diagonal $(+1,-1, \cdots,-1)$.
} 
$\Gamma^{M}$ are $32 \times 32$ imaginary matrices and. In $(9+1)$-dimensions the gamma-matrix which anti commutes with all other gamma matrices is

$$
\gamma^{11}=\left(\begin{array}{cc}
\mathbb{I}_{16 \times 16} & 0 \\
0 & -\mathbb{I}_{16 \times 16}
\end{array}\right)
$$

The chiral projection operator in $(9+1)$-dimensions giving rise to left and right-moving chiral fermions is given by

$$
\mathcal{P}=\frac{1 \pm \gamma^{11}}{2}
$$

Under the chiral projection (A.6), the 32-component Dirac fermions become

$$
\Psi=\left(\begin{array}{c}
\Psi_{L} \\
0
\end{array}\right)
$$

$\Psi$ is a 32 component Majorana-Weyl spinor with 16 non-zero components.

The 32 dimensional $\Gamma$ matrices satisfy the Dirac algebra $\left\{\Gamma^{M}, \Gamma^{N}\right\}=2 \eta^{M N}$. Under the decomposition $\mathrm{SO}(9,1) \rightarrow \mathrm{SO}(1,1) \times \mathrm{SO}(8)$, they have to be written in terms of the 16 dimensional spin(8) matrices. For $M$ corresponding to the $\mathrm{SO}(8)$ directions (that we label by $I$ ) we call the 16 dimensional $\operatorname{spin}(8)$ matrices as $\gamma^{I},(I=1, \ldots, 8)$. The $\gamma^{I}$ 's thus satisfy the $\operatorname{spin}(8)$ algebra $\left\{\gamma^{I}, \gamma^{J}\right\}=2 \delta^{I J}$. They are however reducible and can be written in terms of the 8 dimensional representations $\alpha^{I}$ as,

$$
\gamma^{I}=\left(\begin{array}{cc}
0 & \alpha^{I} \\
\alpha^{I T} & 0
\end{array}\right)
$$

where the $\alpha^{I}$ 's now satisfy $\left\{\alpha^{I}, \alpha^{J}\right\}=2 \delta^{I J}$. A representation of the $\alpha^{I}$ 's can be written follows [41],

$$
\begin{aligned}
& \alpha^{1}=\tau \otimes \tau \otimes \tau \quad \alpha^{2}=1 \otimes \sigma^{1} \otimes \tau \\
& \alpha^{3}=1 \otimes \sigma^{3} \otimes \tau \quad \alpha^{4}=\sigma^{1} \otimes \tau \otimes 1 \\
& \alpha^{5}=\sigma^{3} \otimes \tau \otimes 1 \quad \alpha^{6}=\tau \otimes 1 \otimes \sigma^{1} \\
& \alpha^{7}=\tau \otimes 1 \otimes \sigma^{3} \quad \alpha^{8}=1 \otimes 1 \otimes 1
\end{aligned}
$$

where $\tau=i \sigma^{2}$. We can construct one more $\gamma$ matrix that anti commutes with all the other $\gamma^{I}$ 's. It is given by $\gamma^{9}=\gamma^{1} \gamma^{2} \cdots \gamma^{8}$. In matrix form,

$$
\gamma^{9}=\left(\begin{array}{cc}
1_{8} & 0 \\
0 & -1_{8}
\end{array}\right) \text {. }
$$

The nine 32 dimensional $\Gamma^{M}(M=1, \ldots 9)$ matrices can thus be formed out of the nine $\gamma$ matrices. Since there is no tenth $\gamma$ matrix, we need to construct a tenth $\Gamma$ matrix that anti commutes with all the others. Ultimately we can write,

$$
\begin{aligned}
& \Gamma^{0}=\sigma^{2} \otimes 1_{16} \\
& \Gamma^{I}=i \sigma^{1} \otimes \gamma^{I} \\
& \Gamma^{9}=i \sigma^{1} \otimes \gamma^{9} .
\end{aligned}
$$


With this, the sixteen dimensional $\Psi_{L}$ in equation (A.7) can further be written as,

$$
\Psi_{L}=\left(\begin{array}{c}
\psi_{L} \\
\psi_{R}
\end{array}\right)
$$

where $\psi_{L}$ and $\psi_{R}$ are now 8 component fermions. In other words, the sixteen component $\Psi_{L}$ decomposes as $16=(1,8)+(\overline{1}, \overline{8})$. Thus in $1+1$ dimensions we have 8 one-component left-moving plus 8 one-component right-moving fermions.

We can now write down the dimensionally reduced action,

$$
\begin{aligned}
S_{1+1}= & S_{1+1}^{1}+S_{1+1}^{2} \\
S_{1+1}^{1}= & \frac{1}{g^{2}} \operatorname{tr} \int d^{2} x\left[-\frac{1}{2} F_{\mu \nu} F^{\mu \nu}+D_{\mu} \Phi_{I} D^{\mu} \Phi_{I}+\frac{1}{2}\left[\Phi_{I}, \Phi_{J}\right]^{2}\right] \\
S_{1+1}^{2}= & \frac{i}{g^{2}} \operatorname{tr} \int d^{2} x\left[\psi_{L}^{T} D_{0} \psi_{L}+\psi_{R}^{T} D_{0} \psi_{R}+\psi_{L}^{T} D_{1} \psi_{L}-\psi_{R}^{T} D_{1} \psi_{R}\right. \\
& \left.+2 i \psi_{R}^{T} \alpha_{I}^{T}\left[\Phi_{I}, \psi_{L}\right]\right]
\end{aligned}
$$

where, $D_{\mu}=\partial_{\mu}-i\left[A_{\mu}, \star\right]$ and all the fermions, $\psi_{L}$ and $\psi_{R}$ are anti commuting.

\section{B Tables of fields, eigenfunctions and normalizations}

\begin{tabular}{|c|c|}
\hline \multicolumn{2}{|c|}{ Dimensionfull constants } \\
\hline$q$ & Slope of intersecting brane configuration \\
\hline$\beta$ & Inverse of temperature $T$ \\
\hline$g^{2}$ & Yang-Mills coupling constant \\
\hline \multicolumn{2}{|c|}{ Normalizations } \\
\hline$N$ & $\sqrt{q} / \beta$ \\
\hline $\mathcal{N}(n)$ & $\frac{1}{\sqrt{\pi 2^{n}\left(4 n^{2}-2\right)(n-2) !}}$ \\
\hline $\mathcal{N}(n)$ & $\frac{1}{\sqrt{\pi 2^{n}(4 n-2)(n-1) !}}$ \\
\hline $\mathcal{N}^{\prime}(n)$ & $\frac{1}{\sqrt{\sqrt{\pi} 2^{n} n !}}$ \\
\hline $\mathcal{N}_{F}(n)$ & $\frac{1}{\sqrt{\sqrt{\pi} 2^{n+1}(n-1) !}}$ \\
\hline
\end{tabular}

Table 1. Dimensionfull constants and normalizations.

The various fields together with their eigenfunctions, momenta and momentum modes are tabulated below. 


\begin{tabular}{|c|c|c|c|}
\hline Field & Eigenfunction & $\begin{array}{c}\text { Tree-level } \\
\text { mass }\end{array}$ & $\begin{array}{c}\text { Momentum } \\
\text { mode }\end{array}$ \\
\hline \multicolumn{4}{|c|}{ Bosons } \\
\hline$\left(\begin{array}{c}A_{x}^{2} \\
\phi_{1}^{1}\end{array}\right)$ & $\left(\begin{array}{l}A_{n}(x) \\
\phi_{n}(x)\end{array}\right)=\left(\begin{array}{c}\mathcal{N}(n) e^{-q x^{2} / 2}\left(H_{n}(\sqrt{q} x)+2 n H_{n-2}(\sqrt{q} x)\right) \\
\mathcal{N}(n) e^{-q x^{2} / 2}\left(H_{n}(\sqrt{q} x)-2 n H_{n-2}(\sqrt{q} x)\right)\end{array}\right)$ & $\frac{\lambda_{n}}{g^{2}}=\frac{(2 n-1) q}{g^{2}}$ & $C_{w, n}$ \\
\hline$\left(\begin{array}{l}A_{x}^{2} \\
\phi_{1}^{1}\end{array}\right)$ & $\left(\begin{array}{c}\tilde{A}_{n}(x) \\
\tilde{\phi}_{n}(x)\end{array}\right)=\left(\begin{array}{l}\tilde{\mathcal{N}}(n) e^{-q x^{2} / 2}\left(H_{n}(\sqrt{q} x)-2(n-1) H_{n-2}(\sqrt{q} x)\right) \\
\tilde{\mathcal{N}}(n) e^{-q x^{2} / 2}\left(H_{n}(\sqrt{q} x)+2(n-1) H_{n-2}(\sqrt{q} x)\right)\end{array}\right)$ & $\frac{\tilde{\lambda}_{n}}{g^{2}}=0$ & $\tilde{C}_{w, n}$ \\
\hline$\left(\begin{array}{c}A_{x}^{1} \\
\phi_{1}^{2}\end{array}\right)$ & $\left(\begin{array}{c}-A_{n}(x) \\
\phi_{n}(x)\end{array}\right)=\left(\begin{array}{c}-\mathcal{N}(n) e^{-q x^{2} / 2}\left(H_{n}(\sqrt{q} x)+2 n H_{n-2}(\sqrt{q} x)\right) \\
\mathcal{N}(n) e^{-q x^{2} / 2}\left(H_{n}(\sqrt{q} x)-2 n H_{n-2}(\sqrt{q} x)\right)\end{array}\right)$ & $\frac{\lambda_{n}}{g^{2}}=\frac{(2 n-1) q}{g^{2}}$ & $C_{w, n}^{\prime}$ \\
\hline$\left(\begin{array}{c}A_{x}^{1} \\
\phi_{1}^{2}\end{array}\right)$ & $\left(\begin{array}{c}-\tilde{A}_{n}(x) \\
\tilde{\phi}_{n}(x)\end{array}\right)=\left(\begin{array}{c}-\tilde{\mathcal{N}}(n) e^{-q x^{2} / 2}\left(H_{n}(\sqrt{q} x)-2(n-1) H_{n-2}(\sqrt{q} x)\right) \\
\tilde{\mathcal{N}}(n) e^{-q x^{2} / 2}\left(H_{n}(\sqrt{q} x)+2(n-1) H_{n-2}(\sqrt{q} x)\right)\end{array}\right)$ & $\frac{\tilde{\lambda}_{n}}{g^{2}}=0$ & $\tilde{C}_{w, n}^{\prime}$ \\
\hline $\begin{array}{c}\Phi_{I}^{a},(a=1,2) \\
(I=2 \cdots 8)\end{array}$ & $\mathcal{N}^{\prime}(n) e^{-q x^{2} / 2}\left(H_{n}(\sqrt{q} x)\right)$ & $\frac{\gamma_{n}}{g^{2}}=\frac{(2 n+1) q}{g^{2}}$ & $\Phi_{I}^{a}(w, n)$ \\
\hline $\begin{array}{c}\Phi_{I}^{3} \\
(I=1 \cdots 8)\end{array}$ & $e^{i l x}$ & 0 & $\Phi_{I}^{3}(w, l)$ \\
\hline$A_{x}^{3}$ & $e^{i l x}$ & 0 & $A_{x}^{3}(w, l)$ \\
\hline \multicolumn{4}{|c|}{ Fermions } \\
\hline $\begin{array}{l}\qquad\left(\begin{array}{c}L_{1}^{1} \\
R_{8}^{2}\end{array}\right) \\
\text { and for } 8 \text { other sets }\end{array}$ & $\left(\begin{array}{l}L_{n}(x) \\
R_{n}(x)\end{array}\right)=\left(\begin{array}{l}\mathcal{N}_{F}(n) e^{-q x^{2} / 2}\left(-\frac{i}{\sqrt{2 n}} H_{n}(\sqrt{q} x)+H_{n-1}(\sqrt{q} x)\right) \\
\mathcal{N}_{F}(n) e^{-q x^{2} / 2}\left(-\frac{i}{\sqrt{2 n}} H_{n}(\sqrt{q} x)-H_{n-1}(\sqrt{q} x)\right)\end{array}\right)$ & $\frac{\lambda_{n}^{\prime}}{g^{2}}=\frac{2 n q}{g^{2}}$ & $\theta_{i}(w, n)$ \\
\hline $\begin{array}{l}\qquad\left(\begin{array}{c}L_{1}^{1} \\
R_{8}^{2}\end{array}\right) \\
\text { and for } 8 \text { other sets }\end{array}$ & $\left(\begin{array}{c}L_{n}^{*}(x) \\
R_{n}^{*}(x)\end{array}\right)=\left(\begin{array}{l}\mathcal{N}_{F}(n) e^{-q x^{2} / 2}\left(\frac{i}{\sqrt{2 n}} H_{n}(\sqrt{q} x)+H_{n-1}(\sqrt{q} x)\right) \\
\mathcal{N}_{F}(n) e^{-q x^{2} / 2}\left(\frac{i}{\sqrt{2 n}} H_{n}(\sqrt{q} x)-H_{n-1}(\sqrt{q} x)\right)\end{array}\right)$ & $\frac{\lambda_{n}^{\prime}}{g^{2}}=\frac{2 n q}{g^{2}}$ & $\theta_{i}^{*}(w, n)$ \\
\hline $\begin{array}{l}\qquad\left(\begin{array}{c}L_{1}^{2} \\
R_{8}^{1}\end{array}\right) \\
\text { and for } 8 \text { other sets }\end{array}$ & $\left(\begin{array}{c}L_{n}(x) \\
-R_{n}(x)\end{array}\right)=\left(\begin{array}{c}\mathcal{N}_{F}(n) e^{-q x^{2} / 2}\left(-\frac{i}{\sqrt{2 n}} H_{n}(\sqrt{q} x)+H_{n-1}(\sqrt{q} x)\right) \\
-\mathcal{N}_{F}(n) e^{-q x^{2} / 2}\left(-\frac{i}{\sqrt{2 n}} H_{n}(\sqrt{q} x)-H_{n-1}(\sqrt{q} x)\right)\end{array}\right)$ & $\frac{\lambda_{n}^{\prime}}{g^{2}}=\frac{2 n q}{g^{2}}$ & $\theta_{j}(w, n)$ \\
\hline $\begin{array}{l}\qquad\left(\begin{array}{c}L_{1}^{2} \\
R_{8}^{1}\end{array}\right) \\
\text { and for } 8 \text { other sets }\end{array}$ & $\left(\begin{array}{c}L_{n}^{*}(x) \\
-R_{n}^{*}(x)\end{array}\right)=\left(\begin{array}{c}\mathcal{N}_{F}(n) e^{-q x^{2} / 2}\left(\frac{i}{\sqrt{2 n}} H_{n}(\sqrt{q} x)+H_{n-1}(\sqrt{q} x)\right) \\
-\mathcal{N}_{F}(n) e^{-q x^{2} / 2}\left(\frac{i}{\sqrt{2 n}} H_{n}(\sqrt{q} x)-H_{n-1}(\sqrt{q} x)\right)\end{array}\right)$ & $\frac{\lambda_{n}^{\prime}}{g^{2}}=\frac{2 n q}{g^{2}}$ & $\theta_{j}^{*}(w, n)$ \\
\hline $\begin{array}{c}\quad L_{i}^{3} \\
(i=1 \cdots 8)\end{array}$ & $e^{i l x}$ & 0 & $L_{i}^{3}(w, l)$ \\
\hline $\begin{array}{c}R_{i}^{3} \\
(i=1 \cdots 8)\end{array}$ & $e^{i l x}$ & 0 & $R_{i}^{3}(w, l)$ \\
\hline
\end{tabular}

Table 2. Eigenfunctions, Tree-level masses and momentum modes. 

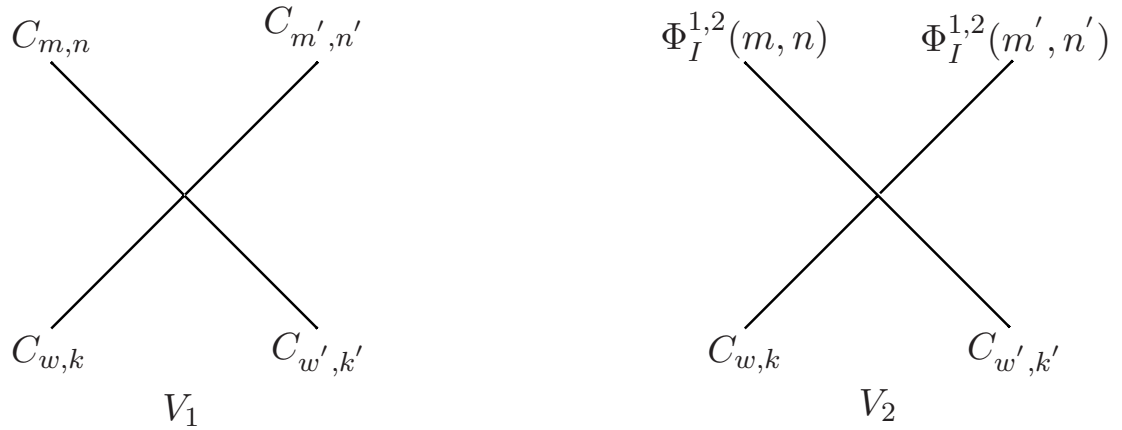

Figure 28. $V_{1}, V_{2}$ vertices.

\section{Propagators and vertices for computation of two-point $C_{w, k}$ ampli- tudes}

\section{C.1 Bosons}

Propagator for $C_{m, n}$

$$
\left\langle C_{m, n} C_{m^{\prime}, n^{\prime}}\right\rangle=g^{2} \frac{\delta_{m,-m^{\prime}} \delta_{n, n^{\prime}}}{\omega_{m}^{2}+\lambda_{n}}
$$

Propagator for $\tilde{C}_{m, n}$

$$
\left\langle\tilde{C}_{m, n} \tilde{C}_{m^{\prime}, n^{\prime}}\right\rangle=g^{2} \frac{\delta_{m,-m^{\prime}} \delta_{n, n^{\prime}}}{\omega_{m}^{2}}
$$

Propagator for the $\Phi_{I}^{1}$ and $\Phi_{I}^{2}$ fluctuations $(I \neq 1)$ are same and is given by

$$
\left\langle\Phi_{I}^{1}(m, n) \Phi_{I}^{1}\left(m^{\prime}, n^{\prime}\right)\right\rangle=g^{2} \frac{\delta_{m,-m^{\prime}} \delta_{n, n^{\prime}}}{\omega_{m}^{2}+\gamma_{n}} .
$$

Propagator for the $\Phi_{I}^{3}$ fluctuations for all $I$ is given by

$$
\left\langle\Phi_{I}^{3}(m, l) \Phi_{I}^{3}\left(m^{\prime}, l^{\prime}\right)\right\rangle=g^{2} \frac{\delta_{m,-m^{\prime}} 2 \pi \delta\left(l+l^{\prime}\right)}{\omega_{m}^{2}+l^{2}} .
$$

Propagator for the $A_{x}^{3}$ fluctuations assumes the form because there is no term in the Lagrangian (2.4) with spatial derivatives on $A_{x}^{3}$.

$$
\begin{aligned}
& \left\langle A_{x}^{3}(m, l) A_{x}^{3}\left(m^{\prime}, l^{\prime}\right)\right\rangle=g^{2} \frac{\delta_{m,-m^{\prime}} 2 \pi \delta\left(l+l^{\prime}\right)}{\omega_{m}^{2}} . \\
& V_{1}=-\frac{N}{2 g^{2}} F_{1}\left(k, k^{\prime}, n, n^{\prime}\right) \delta_{\omega+\omega^{\prime}+m+m^{\prime}} \quad \text { (figure 28) } \\
& F_{1}\left(k, k^{\prime}, n, n^{\prime}\right)=\sqrt{q} \int d x\left[\phi_{k}(x) \phi_{k^{\prime}}(x) A_{n}(x) A_{n^{\prime}}(x)+2 A_{k}(x) \phi_{k^{\prime}}(x) \phi_{n}(x) A_{n^{\prime}}(x)\right. \\
& \left.+2 \phi_{k}(x) A_{k^{\prime}}(x) \phi_{n}(x) A_{n^{\prime}}(x)+A_{k}(x) A_{k^{\prime}}(x) \phi_{n}(x) \phi_{n^{\prime}}(x)\right]
\end{aligned}
$$




$$
\begin{aligned}
& V_{2}=-\frac{N}{2 g^{2}}\left[F_{2}\left(k, k^{\prime}, n, n^{\prime}\right)\right] \delta_{\omega+\omega^{\prime}+m+m^{\prime}} \quad \text { (figure 28) } \\
& F_{2}\left(k, k^{\prime}, n, n^{\prime}\right)=\sqrt{q} \int d x e^{-q x^{2}}\left[A_{k}(x) A_{k^{\prime}}(x)+\phi_{k}(x) \phi_{k^{\prime}}(x)\right]\left[H_{n}(x) H_{n^{\prime}}(x)\right] \\
& \tilde{V}_{1}=-\frac{N}{2 g^{2}} \tilde{F}_{1}\left(k, k^{\prime}, n, n^{\prime}\right) \delta_{\omega+\omega^{\prime}+m+m^{\prime}} \quad \text { (figure 29) } \\
& \tilde{F}_{1}\left(k, k^{\prime}, n, n^{\prime}\right)=\sqrt{q} \int d x\left[\phi_{k}(x) \phi_{k^{\prime}}(x) \tilde{A}_{n}(x) \tilde{A}_{n^{\prime}}(x)+2 A_{k} \phi_{k^{\prime}}(x) \tilde{\phi}_{n}(x) \tilde{A}_{n^{\prime}}(x)\right. \\
& \left.+2 \phi_{k}(x) A_{k^{\prime}}(x) \tilde{\phi}_{n}(x) \tilde{A}_{n^{\prime}}(x)+A_{k}(x) A_{k^{\prime}}(x) \tilde{\phi}_{n}(x) \tilde{\phi}_{n^{\prime}}(x)\right] \\
& V_{2}^{\prime}=-\frac{N}{2 g^{2}}\left[F_{2}^{\prime}\left(k, k^{\prime}, l, l^{\prime}\right)\right] \delta_{\omega+\omega^{\prime}+m+m^{\prime}} \quad \text { (figure 29) } \\
& F_{2}^{\prime}\left(k, k^{\prime}, l, l^{\prime}\right)=\sqrt{q} \int d x\left[A_{k}(x) A_{k^{\prime}}(x)+\phi_{k}(x) \phi_{k^{\prime}}(x)\right]\left[e^{i l x} e^{i l^{\prime} x}\right] \\
& V_{3}=-\frac{N}{2 g^{2}} F_{3}\left(k, k^{\prime}, l, l^{\prime}\right) \delta_{\omega+\omega^{\prime}+m+m^{\prime}} \quad \text { (figure 30) } \\
& F_{3}\left(k, k^{\prime}, l, l^{\prime}\right)=\sqrt{q} \int d x\left[\phi_{k}(x) \phi_{k^{\prime}(x)}\right] e^{i\left(l+l^{\prime}\right) x} \\
& V_{3}^{\prime}=-\frac{N}{2 g^{2}} F_{3}^{\prime}\left(k, k^{\prime}, l, l^{\prime}\right) \delta_{\omega+\omega^{\prime}+m+m^{\prime}} \quad \text { (figure 30) } \\
& F_{3}^{\prime}\left(k, k^{\prime}, l, l^{\prime}\right)=\sqrt{q} \int d x\left[A_{k}(x) A_{k^{\prime}(x)} e^{i l x} e^{i l^{\prime} x}\right] \\
& V_{4}=-\frac{N^{3 / 2}}{g^{2}} F_{4}(k, l, n) \beta \delta_{\omega+m+m^{\prime}} \quad \text { (figure 31) } \\
& F_{4}(k, l, n)=\int d x\left[-\phi_{n}(x) \partial_{x} \phi_{k}(x)+\phi_{k}(x) \partial_{x} \phi_{n}(x)-\phi_{n}(x) A_{k}(x)(q x)\right. \\
& \left.+A_{n}(x) \phi_{k}(x)(q x)\right] e^{i l x} \\
& \tilde{V}_{4}=-\frac{N^{3 / 2}}{g^{2}} \tilde{F}_{4}(k, l, n) \beta \delta_{\omega+m+m^{\prime}} \quad \text { (figure 31) } \\
& \tilde{F}_{4}(k, l, n)=\int d x\left[-\tilde{\phi}_{n}(x) \partial_{x} \phi_{k}(x)+\phi_{k}(x) \partial_{x} \tilde{\phi}_{n}(x)-\tilde{\phi}_{n}(x) A_{k}(x)(q x)\right. \\
& \left.+\tilde{A}_{n}(x) \phi_{k}(x)(q x)\right] e^{i l x} \\
& V_{5}=-\frac{N^{3 / 2}}{2 g^{2}} F_{5}(k, l, n) \beta \delta_{\omega+m+m^{\prime}} \quad \text { (figure 32) } \\
& F_{5}(k, l, n)=\int d x e^{-q x^{2} / 2}\left[e^{i l x} A_{k}(x) \partial_{x} H_{n}(x)-(q x) e^{i l x} A_{k}(x) H_{n}(x)\right. \\
& \left.-(i l) e^{i l x} A_{k}(x) H_{n}(x)+(q x) e^{i l x} \phi_{k}(x) H_{n}(x)\right] \\
& V_{5}^{\prime}=-\frac{N^{3 / 2}}{g^{2}} F_{5}^{\prime}(k, l, n) \beta \delta_{\omega+m+m^{\prime}} \quad \text { (figure 33) } \\
& F_{5}^{\prime}(k, l, n)=\int d x\left[(i l) e^{i l x} \phi_{k}(x) A_{n}(x)+(i l) e^{i l x} A_{k}(x) \phi_{n}(x)\right. \\
& \left.-e^{i l x} \partial_{x} \phi_{k}(x) A_{n}(x)-e^{i l x} \partial_{x} \phi_{n}(x) A_{k}(x)\right]
\end{aligned}
$$



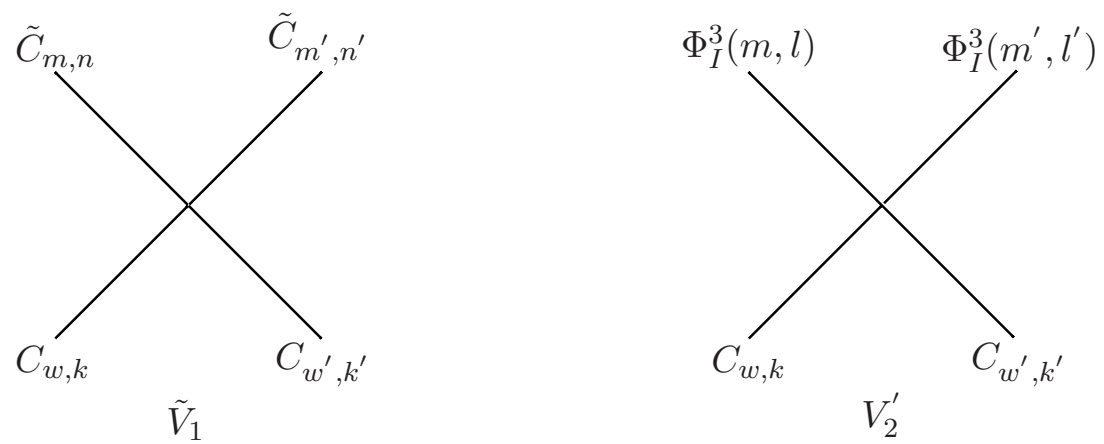

Figure 29. $\tilde{V}_{1}, V_{2}^{\prime}$ vertices.

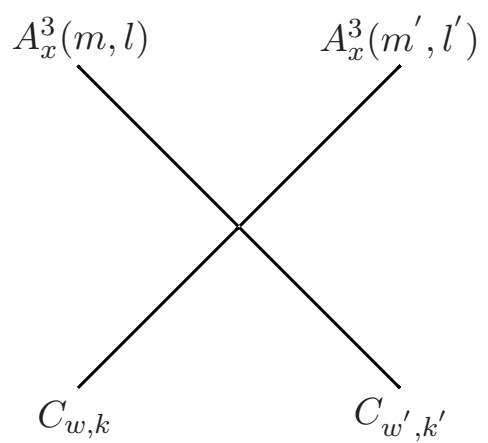

$V_{3}$

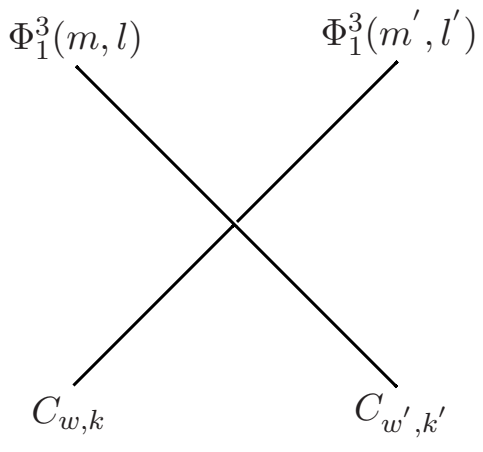

$V_{3}^{\prime}$

Figure 30. $V_{3}$ and $V_{3}^{\prime}$ vertices.
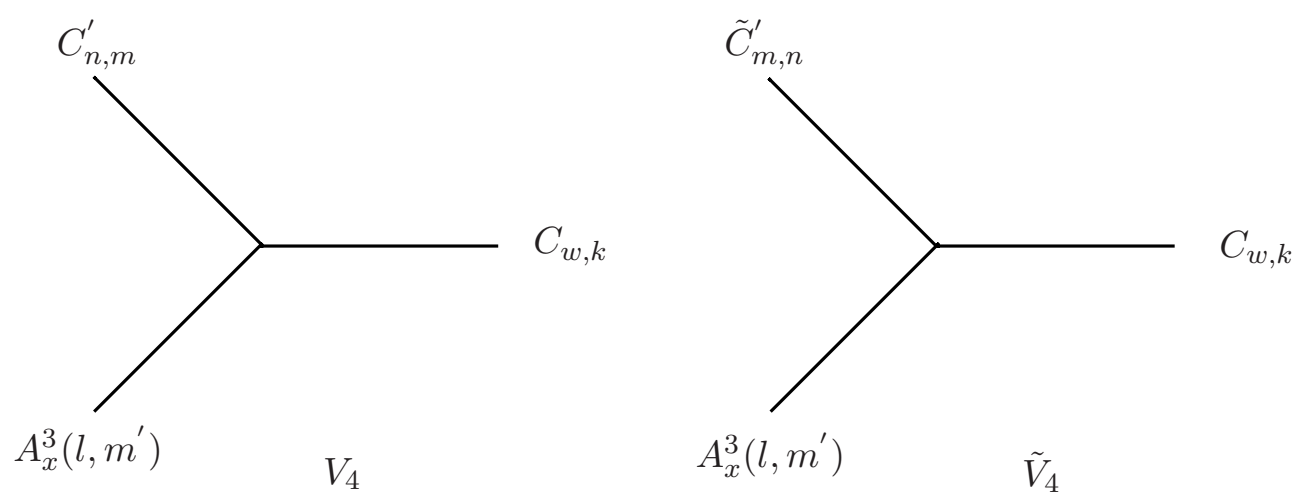

Figure 31. $V_{4}$ and $\tilde{V}_{4}$ vertices.

$$
\begin{aligned}
\tilde{V}_{5}^{\prime}= & -\frac{N^{3 / 2}}{g^{2}} \tilde{F}_{5}^{\prime}(k, l, n) \beta \delta_{\omega+m+m^{\prime}} \quad \text { (figure 33) } \\
\tilde{F}_{5}^{\prime}(k, l, n)= & \int d x\left[(i l) e^{i l x} \phi_{k}(x) \tilde{A}_{n}(x)+(i l) e^{i l x} A_{k}(x) \tilde{\phi}_{n}(x)\right. \\
& \left.-e^{i l x} \partial_{x} \phi_{k}(x) \tilde{A}_{n}(x)-e^{i l x} \partial_{x} \tilde{\phi}_{n}(x) A_{k}(x)\right]
\end{aligned}
$$




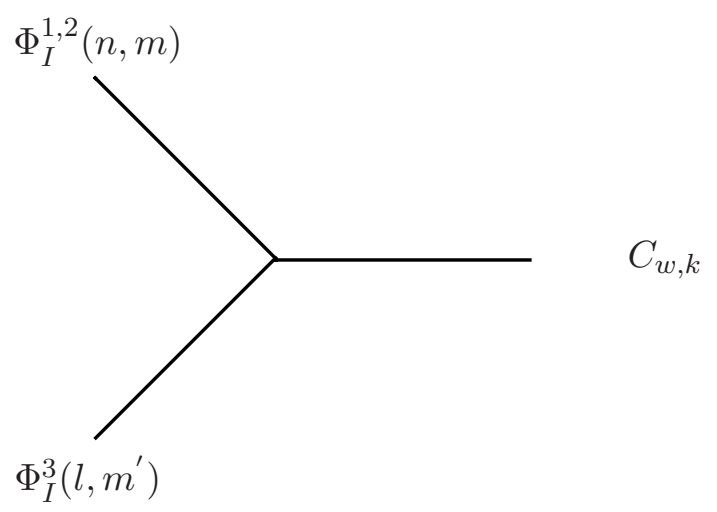

$V_{5}$

Figure 32. $V_{5}$ vertex.
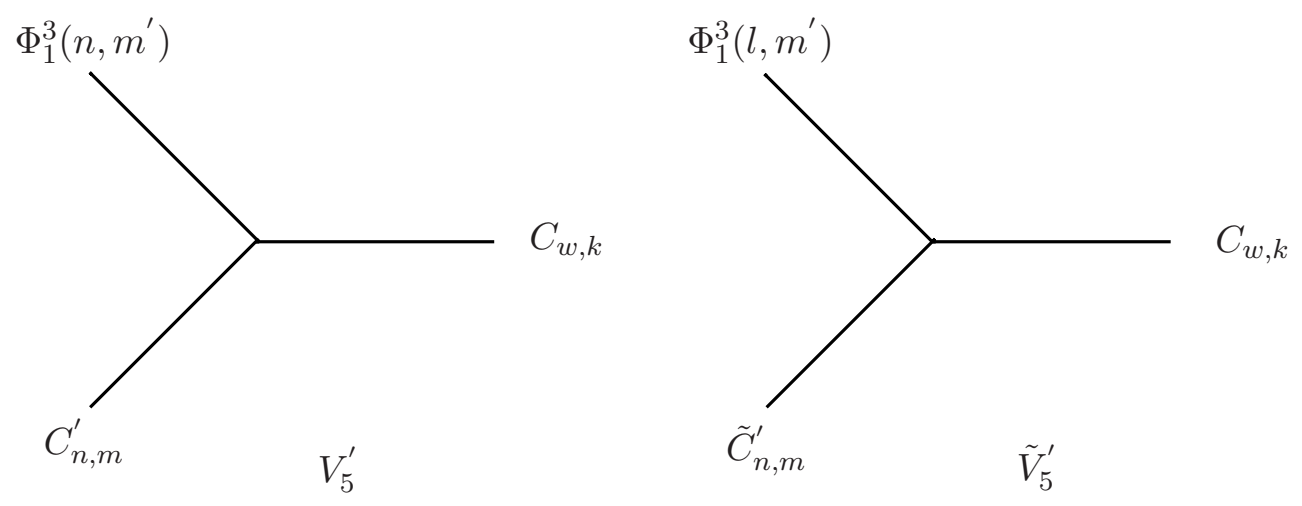

Figure 33. $V_{5}^{\prime}$ and $\tilde{V}_{5}^{\prime}$ vertices.

\section{C.2 Fermions}

Propagator for the $L_{i}^{a}$ and $R_{i}^{a}$ modes $(a=1,2)$,

$$
\left\langle\theta_{j}(m, n) \theta_{k}^{*}\left(m^{\prime}, n^{\prime}\right)\right\rangle=\frac{g^{2}}{N^{1 / 2}} \frac{\delta_{j k} \delta_{m, m^{\prime}} \delta_{n^{\prime} n^{\prime}}}{i \omega_{m}+\sqrt{\lambda_{n}^{\prime}}}
$$

$\lambda_{n}^{\prime}=2 n q$.

Propagator for the $L_{i}^{3}$ and $R_{i}^{3}$ modes.

$$
\begin{aligned}
\left\langle L_{i}^{3}(m, l) L_{k}^{3}\left(m^{\prime}, l^{\prime}\right)\right\rangle & =\frac{g^{2}}{N^{1 / 2}} \frac{\delta_{i k} \delta_{m,-m^{\prime}} 2 \pi \delta\left(l+l^{\prime}\right)}{i \omega_{m}+l} \\
\left\langle R_{i}^{3}(m, l) R_{k}^{3}\left(m^{\prime}, l^{\prime}\right)\right\rangle & =\frac{g^{2}}{N^{1 / 2}} \frac{\delta_{i k} \delta_{m,-m^{\prime}} 2 \pi \delta\left(l+l^{\prime}\right)}{i \omega_{m}-l} \\
V_{6}^{R / L} & =i \frac{N}{g^{2}} F_{6}^{R / L}(k, n, l) \delta_{w+m+m^{\prime}} \quad \text { (figure 34). }
\end{aligned}
$$

Where,

$$
F_{6}^{R}(k, n, l)=\sqrt{q} \int d x \phi_{k}(x) R_{n}(x) e^{i l x}
$$



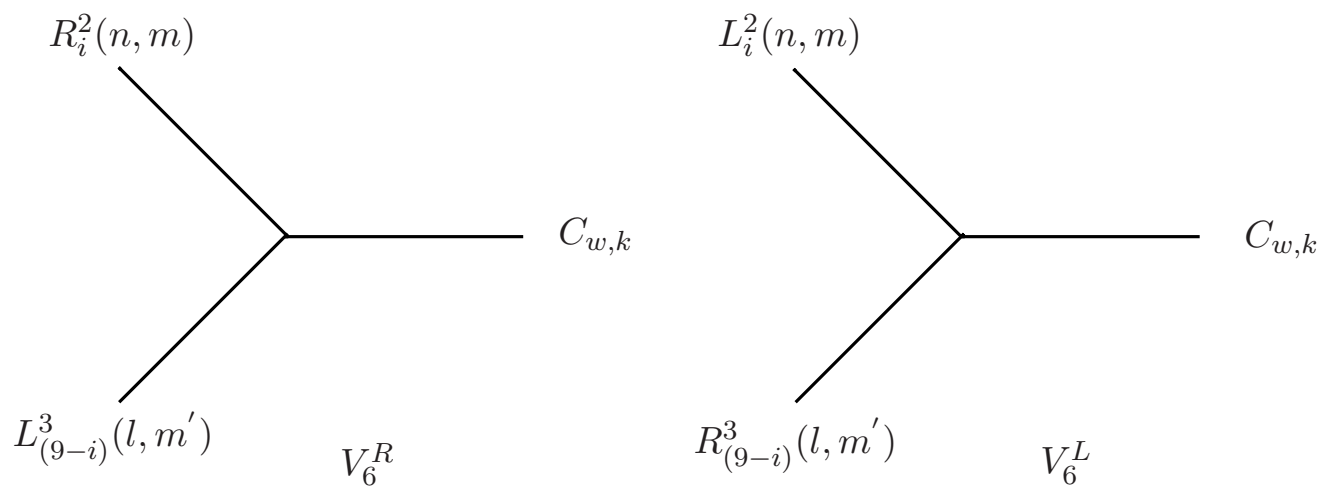

Figure 34. $V_{6}^{R}$ and $V_{6}^{L}$ vertices.
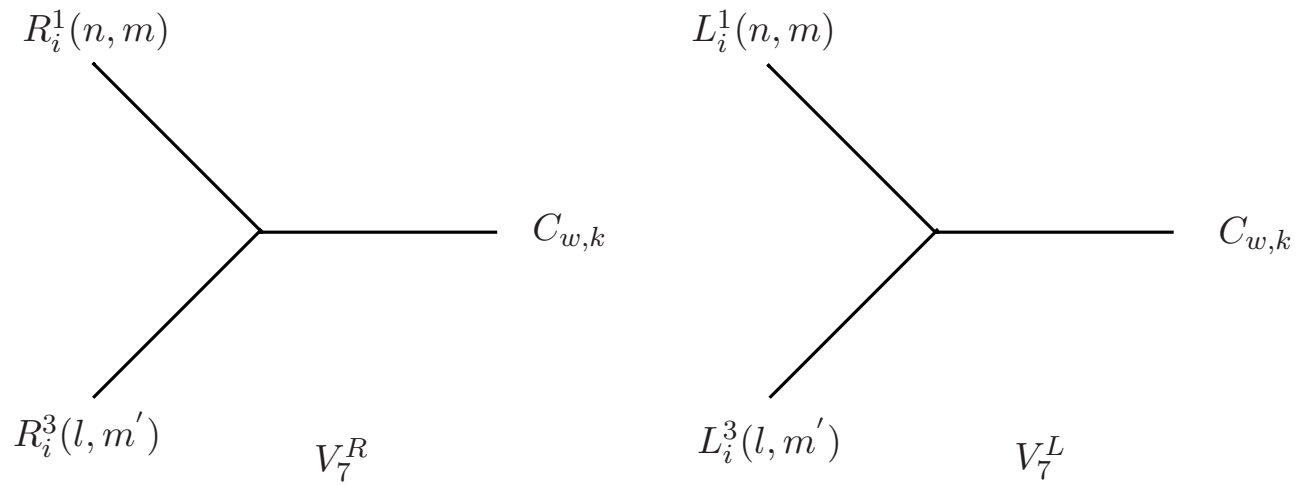

Figure 35. $V_{7}^{R}$ and $V_{7}^{L}$ vertices.

There are eight such vertices involving $R_{i}^{2}$ and $L_{(9-i)}^{3}(i=1 \cdots 8)$. We also have eight vertices involving $L_{i}^{2}$ and $R_{(9-i)}^{3}(i=1 \cdots 8)$. The corresponding vertices would be given by replacing $R_{n}(x)$ with $L_{n}(x)$. This vertex is thus,

$$
F_{6}^{L}(k, n, l)=\mp \sqrt{q} \int d x \phi_{k}(x) L_{n}(x) e^{i l x} .
$$

The $\mp$ is due to the fact that half of the above vertices come with sign opposite to that of the other half in the Lagrangian. There is no $\mp$ in (C.31) as the minus sign coming from the vertices in the Lagrangian is compensated by the ones from the eigenfunctions.

Similarly the other eight vertices involving both $R_{i}^{3}$ and $R_{i}^{a}$ with $(a=1,2)$ (or the $L$ legs) have the same structure.

$$
\begin{aligned}
V_{7}^{R / L} & =i \frac{N}{g^{2}} F_{7}^{R / L}(k, n, l) \delta_{w+m+m^{\prime}} \quad \text { (figure 35) } \\
F_{7}^{R}(k, n, l) & =\mp \sqrt{q} \int d x A_{k}(x) R_{n}(x) e^{i l x} \\
F_{7}^{L}(k, n, l) & =\sqrt{q} \int d x A_{k}(x) L_{n}(x) e^{i l x} .
\end{aligned}
$$

Here the $\mp$ in the expression for $F_{7}^{R}(k, n, l)$ is due to the fact that half of the eigenfunctions come with a sign opposite to the other half and in the Lagrangian all the terms come with the same sign. 

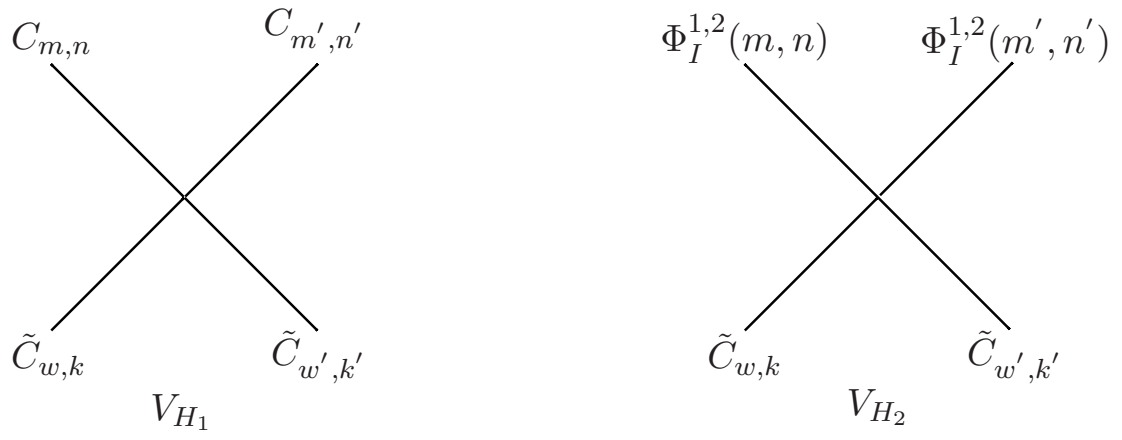

Figure 36. $V_{H_{1}}, V_{H_{2}}$ vertices.

\section{Vertices for computation of two-point $\tilde{C}_{w, k}$ amplitudes}

Since the zero-eigenfunctions are also massless fields we compute their two-point finite temperature amplitudes. The amplitudes are given in section (5.3). Here we present the various four-point and three-point vertices that occur in the calculation for the amplitudes of the zero-eigenfunctions.

\section{D.1 Bosonic vertices}

$$
\begin{aligned}
V_{H_{1}}= & -\frac{N}{2 g^{2}} H_{1}\left(k, k^{\prime}, n, n^{\prime}\right) \delta_{\omega+\omega^{\prime}+m+m^{\prime}} \quad(\text { figure } 36) \\
H_{1}\left(k, k^{\prime}, n, n^{\prime}\right)= & \sqrt{q} \int d x\left[\tilde{\phi}_{k}(x) \tilde{\phi}_{k^{\prime}}(x) A_{n}(x) A_{n^{\prime}}(x)+2 \tilde{A}_{k}(x) \tilde{\phi}_{k^{\prime}}(x) \phi_{n}(x) A_{n^{\prime}}(x)\right. \\
& \left.+2 \tilde{\phi}_{k}(x) \tilde{A}_{k^{\prime}}(x) \phi_{n}(x) A_{n^{\prime}}(x)+\tilde{A}_{k}(x) \tilde{A}_{k^{\prime}}(x) \phi_{n}(x) \phi_{n^{\prime}}(x)\right] \\
V_{H_{2}}= & -\frac{N}{2 g^{2}}\left[H_{2}\left(k, k^{\prime}, n, n^{\prime}\right)\right] \delta_{\omega+\omega^{\prime}+m+m^{\prime}} \\
H_{2}\left(k, k^{\prime}, n, n^{\prime}\right)= & \sqrt{q} \int d x e^{-q x^{2}}\left[\tilde{A}_{k}(x) \tilde{A}_{k^{\prime}}(x)+\tilde{\phi}_{k}(x) \tilde{\phi}_{k^{\prime}}(x)\right] \\
& \times\left[H_{n}(\sqrt{q} x) H_{n^{\prime}}(\sqrt{q} x)\right] \\
\tilde{H}_{1}\left(k, k^{\prime}, n, n^{\prime}\right)= & \sqrt{q} \int d x\left[\tilde{\phi}_{k}(x) \tilde{\phi}_{k^{\prime}}(x) \tilde{A}_{n}(x) \tilde{A}_{n^{\prime}}(x)+2 \tilde{A}_{k}(x) \tilde{\phi}_{k^{\prime}}(x) \tilde{\phi}_{n}(x) \tilde{A}_{n^{\prime}}(x)\right. \\
& \left.+2 \tilde{\phi}_{k}(x) \tilde{A}_{k^{\prime}}(x) \tilde{\phi}_{n}(x) \tilde{A}_{n^{\prime}}(x)+\tilde{A}_{k}(x) \tilde{A}_{k^{\prime}}(x) \tilde{\phi}_{n}(x) \tilde{\phi}_{n^{\prime}}(x)\right] \\
V_{H_{2}}^{\prime}= & -\frac{N}{2 g^{2}}\left[H_{2}^{\prime}\left(k, k^{\prime}, l, l^{\prime}\right)\right] \delta_{\omega+\omega^{\prime}+m+m^{\prime}} \quad(\text { figure } 37) \\
H_{2}^{\prime}\left(k, k^{\prime}, l, l^{\prime}\right)= & \sqrt{q} \int d x\left[\tilde{A}_{k}(x) \tilde{A}_{k^{\prime}}(x)+\tilde{\phi}_{k}(x) \tilde{\phi}_{k^{\prime}}(x)\right]\left[e^{i l x} e^{i l^{\prime} x}\right] \\
V_{H_{3}}= & -\frac{N}{2 g^{2}} H_{3}\left(k, k^{\prime}\right) \delta_{\omega+\omega^{\prime}+m+m^{\prime}} \quad(\text { figure } 38) \\
H_{3}\left(k, k^{\prime} l, l^{\prime}\right)= & \sqrt{q} \int d x\left[\tilde{\phi}_{k}(x) \tilde{\phi}_{k^{\prime}(x)}\right] e^{i l x} e^{i l}{ }^{\prime} x
\end{aligned}
$$




$$
\begin{aligned}
& V_{H_{3}}^{\prime}=-\frac{N}{2 g^{2}} H_{3}^{\prime}\left(k, k^{\prime}, l, l^{\prime}\right) \delta_{\omega+\omega^{\prime}+m+m^{\prime}} \quad \text { (figure 38) } \\
& H_{3}^{\prime}\left(k, k^{\prime}, l, l^{\prime}\right)= \sqrt{q} \int d x\left[\tilde{A}_{k}(x) \tilde{A}_{k^{\prime}(x)} e^{i l x} e^{i l^{\prime} x}\right] \\
& V_{H_{4}}=-\frac{N^{3 / 2}}{g^{2}} H_{4}(k, l, n) \beta \delta_{\omega+m+m^{\prime}} \quad \text { (figure 39) } \\
& H_{4}(k, l, n)= \int d x\left[-\phi_{n}(x) \partial_{x} \tilde{\phi}_{k}(x)+\tilde{\phi}_{k}(x) \partial_{x} \phi_{n}(x)-\phi_{n}(x) \tilde{A}_{k}(x)(q x)\right. \\
&\left.+\tilde{\phi}_{k}(x) A_{n}(x)(q x)\right] e^{i l x} \\
& \tilde{V}_{H_{4}}=-\frac{N^{3 / 2}}{g^{2}} \tilde{H}_{4}(k, l, n) \beta \delta_{\omega+m+m^{\prime}} \quad(\text { figure } 39) \\
& \tilde{H}_{4}(k, l, n)= \int d x\left[-\tilde{\phi}_{n}(x) \partial_{x} \tilde{\phi}_{k}(x)+\tilde{\phi}_{k}(x) \partial_{x} \tilde{\phi}_{n}(x)-\tilde{\phi}_{n}(x) \tilde{A}_{k}(x)(q x)\right. \\
&\left.+\tilde{\phi}_{k}(x) \tilde{A}_{n}(x)(q x)\right] e^{i l x} \\
& V_{H_{5}}=-\frac{N^{3 / 2}}{2 g^{2}} H_{5}(k, l, n) \beta \delta_{\omega+m+m^{\prime}} \quad(\text { figure } 40) \\
& H_{5}(k, l, n)= \int d x e^{-q x^{2} / 2}\left[e^{i l x} \tilde{A}_{k}(x) \partial_{x} H_{n}(\sqrt{q} x)-(q x) e^{i l x} \tilde{A}_{k}(x) H_{n}(\sqrt{q} x)\right. \\
&\left.-(i l) e^{i l x} \tilde{A}_{k}(x) H_{n}(\sqrt{q} x)+(q x) e^{i l x} \tilde{\phi}_{k}(x) H_{n}(\sqrt{q} x)\right] \\
& \tilde{V}_{H_{5}}{ }^{\prime}=-\frac{N^{3 / 2}}{g^{2}} \tilde{H}_{5}^{\prime}(k, l, n) \beta \delta_{\omega+m+m^{\prime}} \quad(\text { figure } 41) \\
& \tilde{H}_{5}^{\prime}(k, l, n)= \int d x\left[(i l) e^{i l x} \tilde{\phi}_{k}(x) \tilde{A}_{n}(x)+(i l) e^{i l x} \tilde{A}_{k}(x) \tilde{\phi}_{n}(x)\right. \\
& V_{H_{5}}^{\prime}(k, l, n)=-\frac{N^{3 / 2}}{g^{2}} H_{5}^{\prime}(k, l, n) \beta \delta_{\omega+m+m^{\prime}} \quad(\text { figure } 41) \\
& \int(i l) e_{x}^{i l x} \tilde{\phi}_{k}(x) A_{n}(x)+(i l) e^{i l x} \tilde{A}_{k}(x) \phi_{n}(x) \\
&\left.\tilde{\phi}_{k}(x) \tilde{A}_{n}(x)-e^{i l x} \partial_{x} \tilde{\phi}_{n}(x) \tilde{A}_{k}(x)\right]
\end{aligned}
$$

\section{D.2 Fermionic vertices}

The fermionic three-point vertices for the $\tilde{C}_{w, k}-\tilde{C}_{w^{\prime}, k^{\prime}}$ two-point functions at finite temperature participate in the cancellation of UV divergence as in the case of the tachyonic amplitudes. The propagators for the fermionic loops are given in appendix (C.2). The various three-point vertices are given below.

$$
\begin{aligned}
\tilde{V}_{H_{6}}^{R / L} & =i \frac{N}{g^{2}} \tilde{H}_{6}^{R / L}(k, n, l) \delta_{w+m+m^{\prime}} \quad \text { (figure 42) } \\
\tilde{H}_{6}^{R}(k, n, l) & =\sqrt{q} \int d x \tilde{\phi}_{k}(x) R_{n}(x) e^{i l x}
\end{aligned}
$$



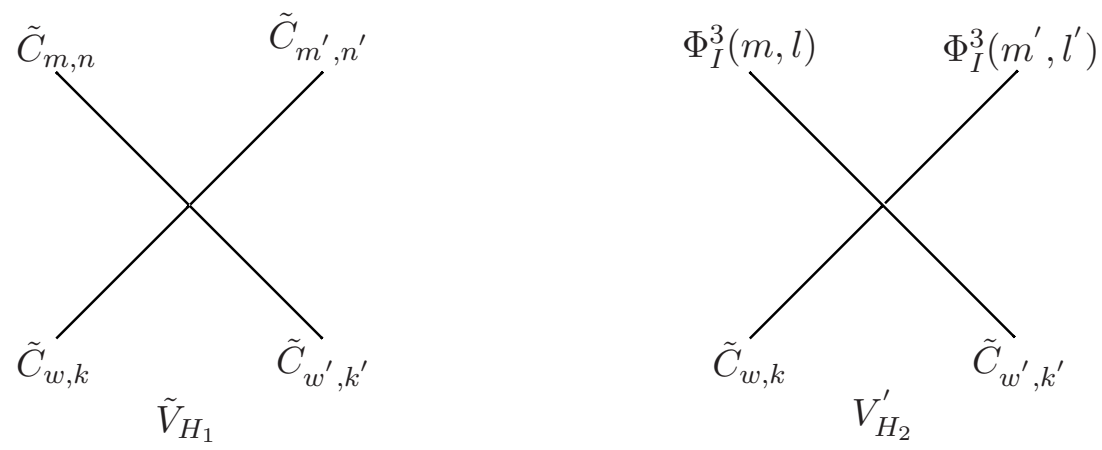

Figure 37. $\tilde{V}_{H_{1}}, V_{H_{2}}^{\prime}$ vertices.

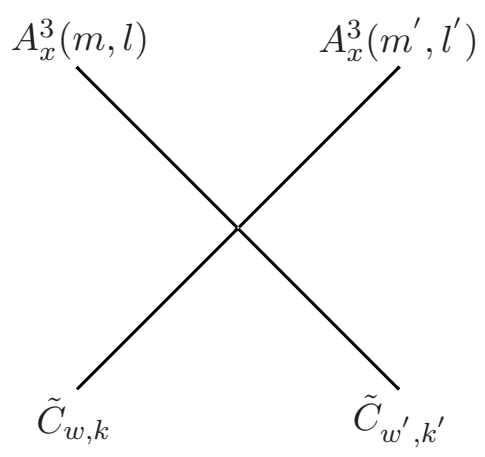

$V_{H_{3}}$

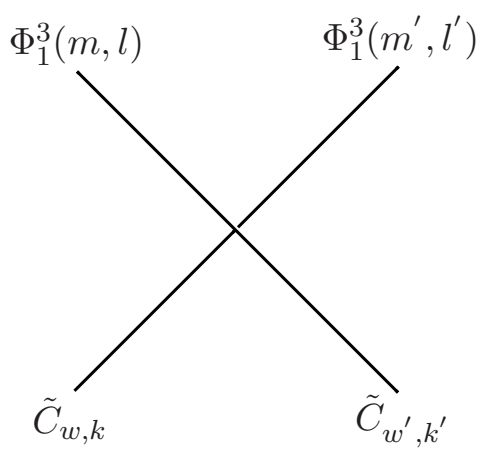

$V_{H_{3}}^{\prime}$

Figure 38. $V_{H_{3}}$ and $V_{H_{3}}^{\prime}$ vertices.
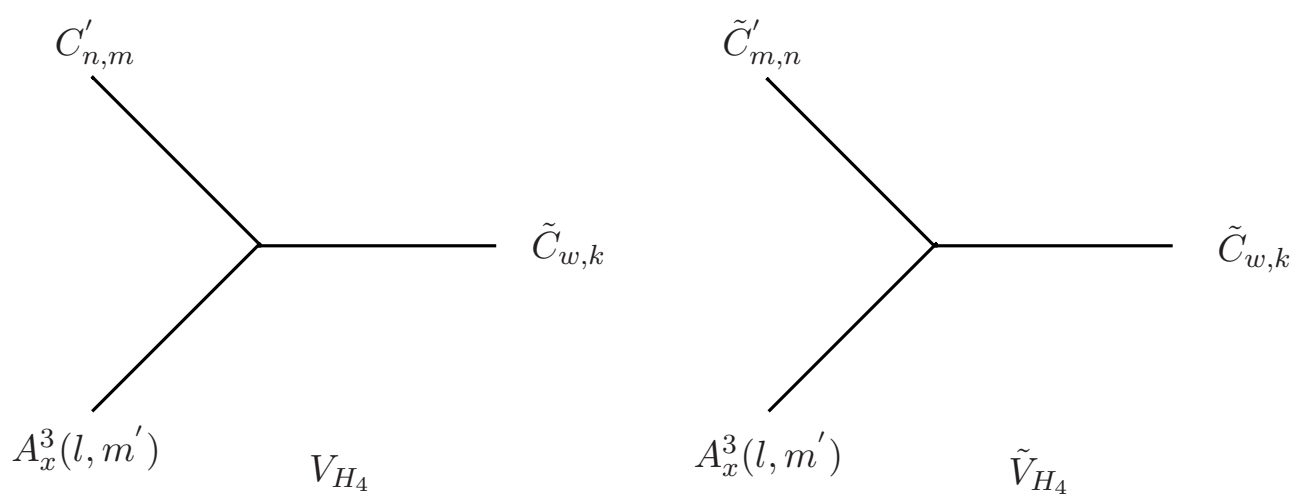

Figure 39. $V_{H_{4}}$ and $\tilde{V}_{H_{4}}$ vertices.

$$
\begin{aligned}
\tilde{H}_{6}^{L}(k, n, l) & =\mp \sqrt{q} \int d x \tilde{\phi}_{k}(x) L_{n}(x) e^{i l x} \\
\tilde{V}_{H_{7}}^{R / L} & =i \frac{N}{g^{2}} \tilde{H}_{7}^{R / L}(k, n, l) \delta_{w+m+m^{\prime}} \quad \text { (figure 43) } \\
\tilde{H}_{7}^{R}(k, n, l) & =\mp \sqrt{q} \int d x \tilde{A}_{k}(x) R_{n}(x) e^{i l x} \\
\tilde{H}_{7}^{L}(k, n, l) & =\sqrt{q} \int d x \tilde{A}_{k}(x) L_{n}(x) e^{i l x} .
\end{aligned}
$$




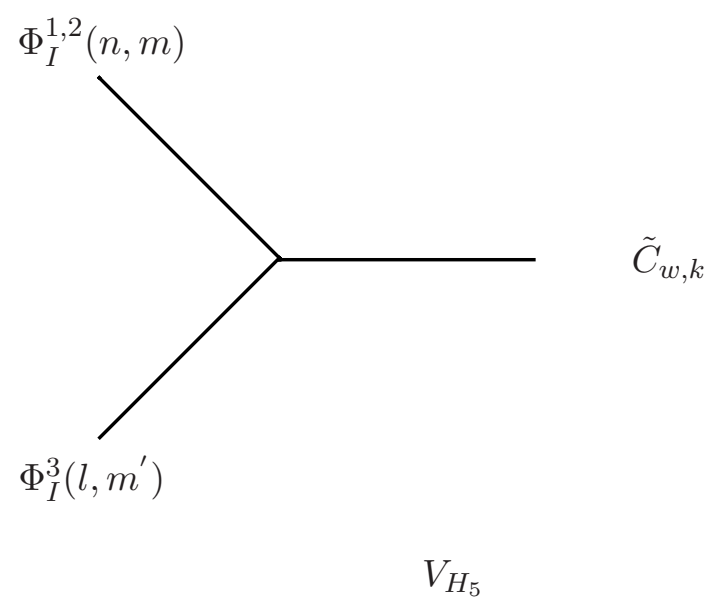

Figure 40. $V_{H_{5}}$ vertex.
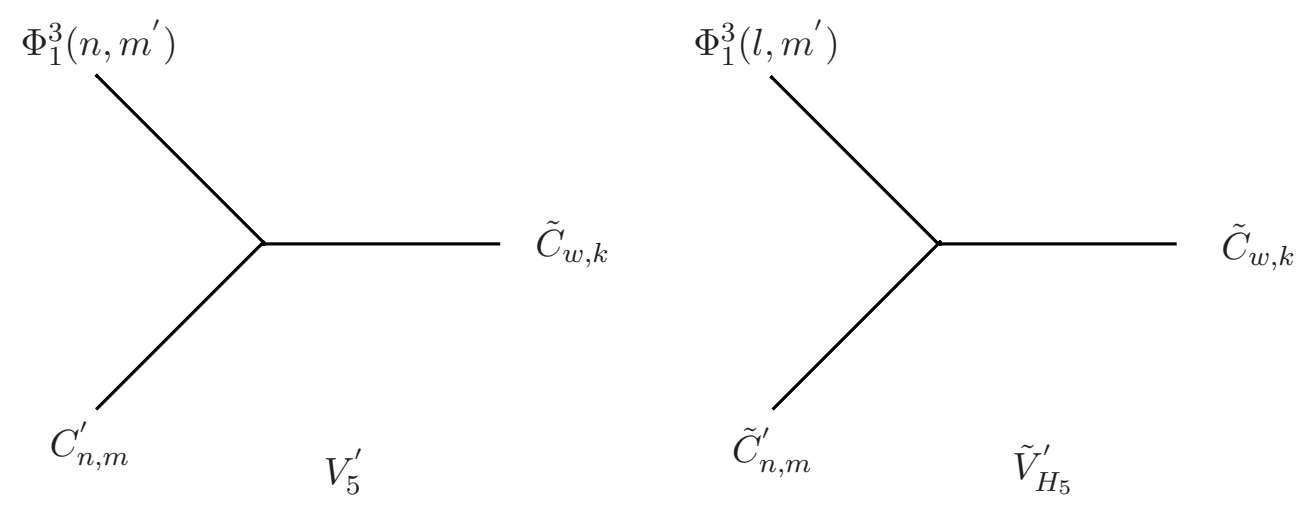

Figure 41. $V_{H_{5}}^{\prime}$ and $\tilde{V}_{H_{5}}^{\prime}$ vertices.
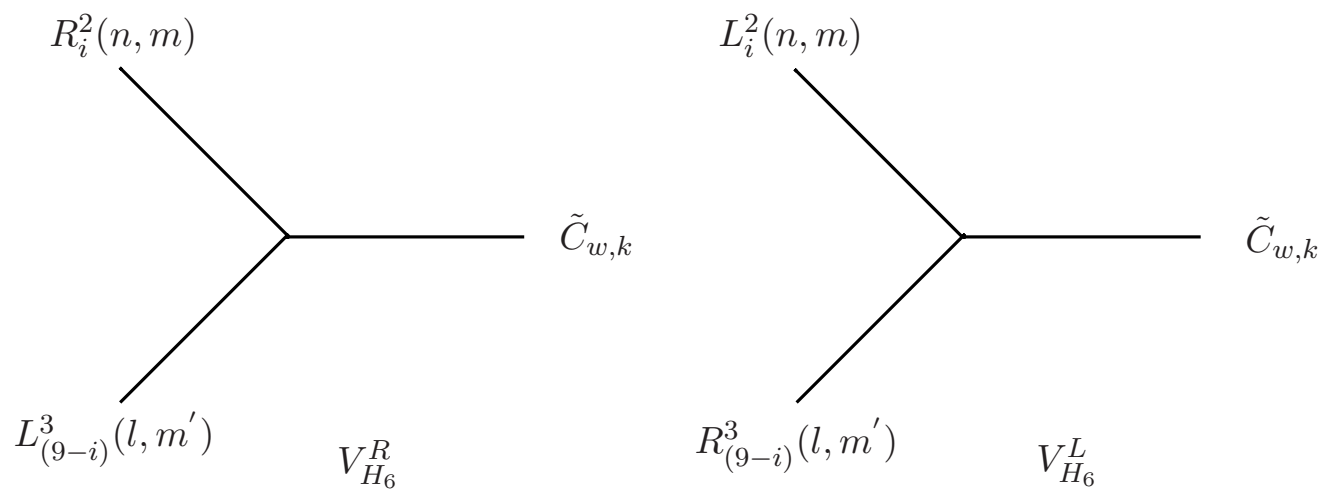

Figure 42. $V_{H_{6}}^{R}$ and $V_{H_{6}}^{L}$ vertices. 

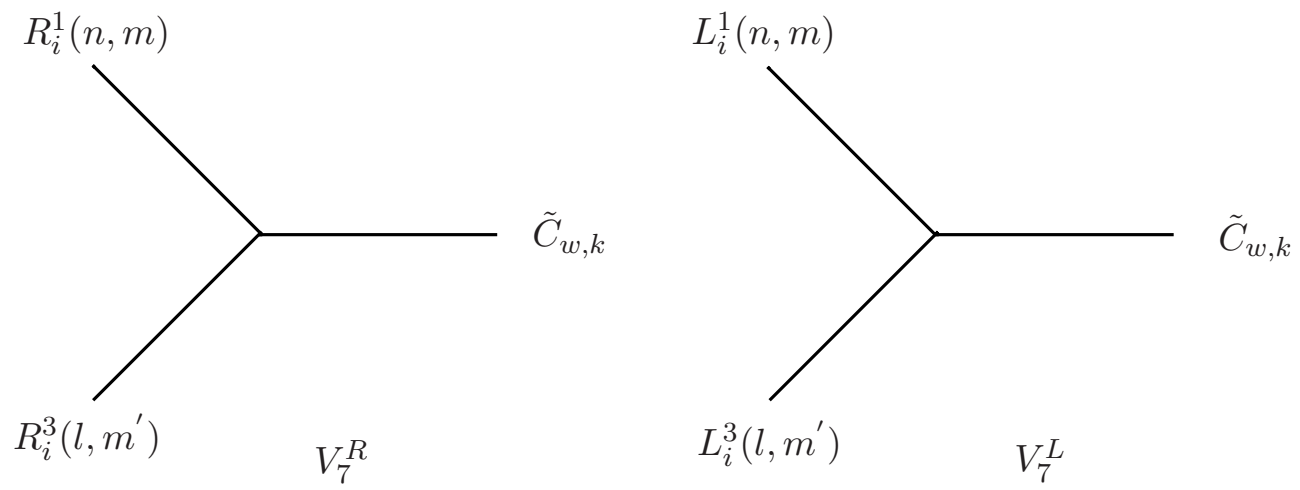

Figure 43. $V_{H_{7}}^{R}$ and $V_{H_{7}}^{L}$ vertices.
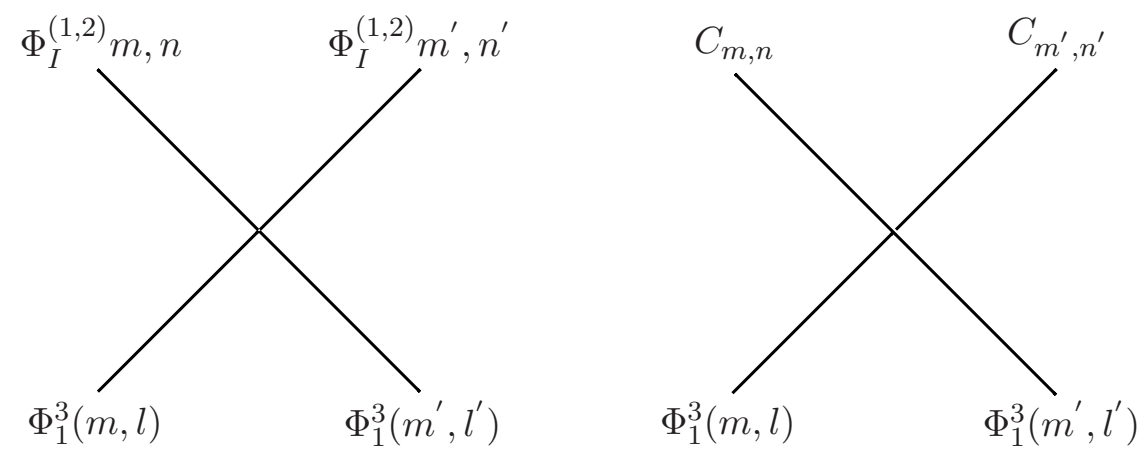

$V_{1}^{1}$

$V_{2}^{1}$

Figure 44. $V_{1}^{1}$ and $V_{2}^{1}$ vertices.

The origin of the $\mp$ sign is explained in appendix C.2.

\section{E $\quad$ Vertices for computation of two point amplitudes for $\Phi_{I}^{3}$ and $A_{x}^{3}$}

\section{E.1 $\Phi_{1}^{3}$ vertices}

We list here all the four-point and three-point bosonic and three-point fermionic vertices that constitute the two-point functions for $\Phi_{1}^{3}-\Phi_{1}^{3}$.

$$
\begin{array}{rlr}
V_{1}^{1} & =-\frac{N}{2 g^{2}} G_{1}^{1}\left(l, l^{\prime}, n, n^{\prime}\right) \delta_{w+w^{\prime}+m+m^{\prime}} & \text { (figure 44) } \\
G_{1}^{1}\left(l, l^{\prime}, n, n^{\prime}\right) & =\sqrt{q} \int d x e^{-q x^{2}} H_{n}(x) H_{n^{\prime}}(x) e^{i l x} e^{i l^{\prime} x} & \\
V_{2}^{1} & =-\frac{N}{2 g^{2}} G_{2}^{1}\left(l, l^{\prime}, n, n^{\prime}\right) \delta_{w+w^{\prime}+m+m^{\prime}} \quad \text { (figure 44) } \\
G_{2}^{1}\left(l, l^{\prime}, n, n^{\prime}\right) & =\sqrt{q} \int d x A_{n}(x) A_{n^{\prime}}(x) e^{i l x} e^{i l^{\prime} x} & \\
\tilde{V}_{2}^{1} & =-\frac{N}{2 g^{2}} \tilde{G}_{2}^{1}\left(l, l^{\prime}, n, n^{\prime}\right) \delta_{w+w^{\prime}+m+m^{\prime}} & \text { (figure 45) } \\
\tilde{G}_{2}^{1}\left(l, l^{\prime}, n, n^{\prime}\right) & =\sqrt{q} \int d x \tilde{A}_{n}(x) \tilde{A}_{n^{\prime}}(x) e^{i l x} e^{i l^{\prime} x} &
\end{array}
$$




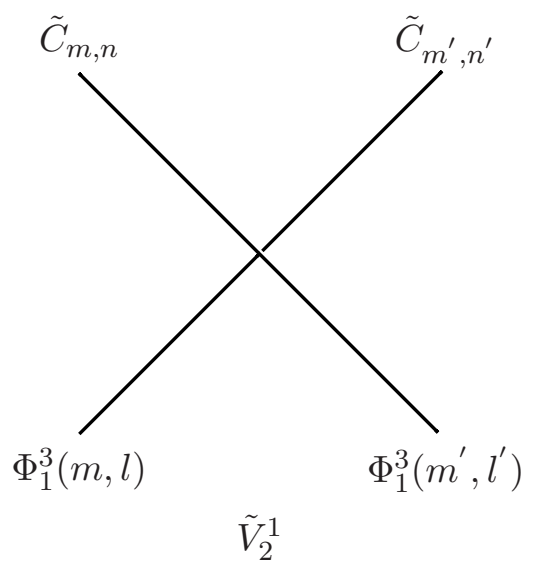

Figure 45. $\tilde{V}_{2}^{1}$ vertex.

$$
\begin{aligned}
V_{1}^{1^{\prime}}= & -\frac{N}{2 g^{2}} G_{1}^{1^{\prime}}\left(l, n, n^{\prime}\right) \delta_{w+m+m^{\prime}} \quad \text { (figure 46) } \\
G_{1}^{1^{\prime}}\left(l, n, n^{\prime}\right)= & \sqrt{q} \int d x\left[q x e^{-q x^{2}} H_{n}(x) H_{n^{\prime}}(x) e^{i l x}\right] \\
V_{3}^{1}= & -\frac{N^{3 / 2}}{g^{2}} G_{3}^{1}\left(l, n, n^{\prime}\right) \beta \delta_{w+m+m^{\prime}} \quad \text { (figure 46) } \\
G_{3}^{1}\left(l, n, n^{\prime}\right)= & \int d x e^{i l x}\left[q x A_{n}(x) A_{n^{\prime}}(x)+\partial_{x} \phi_{n^{\prime}}(x) A_{n}(x)-i l A_{n}(x) \phi_{n^{\prime}}(x)\right] \\
\tilde{V}_{3}^{1}= & -\frac{N^{3 / 2}}{g^{2}} \tilde{G}_{3}^{1}\left(l, n, n^{\prime}\right) \beta \delta_{w+m+m^{\prime}} \quad(\text { figure } 47) \\
\tilde{G}_{3}^{1}\left(l, n, n^{\prime}\right)= & \int d x e^{i l x}\left[q x \tilde{A}_{n}(x) \tilde{A}_{n^{\prime}}(x)+\partial_{x} \tilde{\phi}_{n^{\prime}}(x) \tilde{A}_{n}(x)-i l \tilde{A}_{n}(x) \tilde{\phi}_{n^{\prime}}(x)\right] \\
\tilde{V}_{3}^{1^{\prime}}= & -\frac{N^{3 / 2}}{g^{2}} \tilde{G}_{3}^{1^{\prime}}\left(l, n, n^{\prime}\right) \beta \delta_{w+m+m^{\prime}} \quad(\text { figure } 47) \\
\tilde{G}_{3}^{1^{\prime}}\left(l, n, n^{\prime}\right)= & \int d x e^{i l x}\left[2 q x A_{n}(x) \tilde{A}_{n^{\prime}}(x)+\partial_{x} \tilde{\phi}_{n^{\prime}}(x) A_{n}(x)-i l A_{n}(x) \tilde{\phi}_{n^{\prime}}(x)\right. \\
& \left.+\partial_{x} \phi_{n}(x) \tilde{A}_{n^{\prime}}(x) e^{i l x}-i l \tilde{A}_{n^{\prime}}(x) \phi_{n}(x)\right] \\
V_{f}^{1}= & i \frac{N}{g^{2}} G_{f}^{1}\left(l, n, n^{\prime}\right) \delta_{w+m+m^{\prime}} \quad(\text { figure } 48) \\
G_{f}^{2}\left(l, n, n^{\prime}\right)= & \sqrt{q} \int d x e^{i l x}\left[R_{n}(x) L_{n^{\prime}}^{*}-L_{n}(x) R_{n^{\prime}}^{*}(x)\right] \\
V_{f}^{2}= & i \frac{N}{g^{2}} G_{f}^{2}\left(l, n, n^{\prime}\right) \delta_{w+m+m^{\prime}} \quad(\text { figure } 48) \\
\left.G^{\prime}\right)= & \sqrt{q} \int d x e^{i l x} R_{n}(x) L_{n^{\prime}}(x)
\end{aligned}
$$



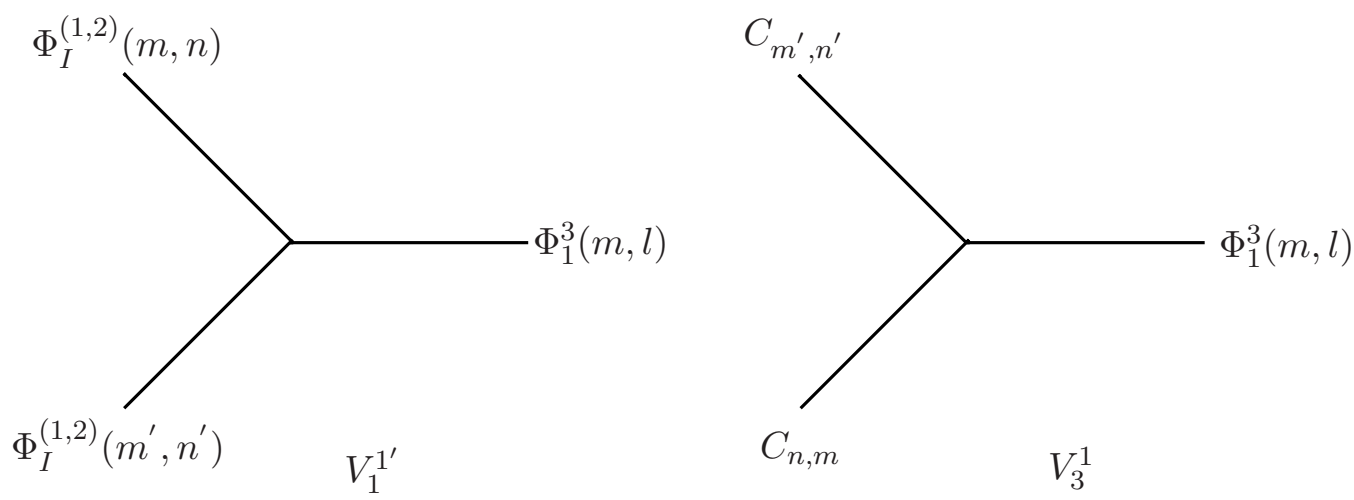

Figure 46. $V_{1}^{1^{\prime}}$ and $V_{3}^{1}$ vertices.
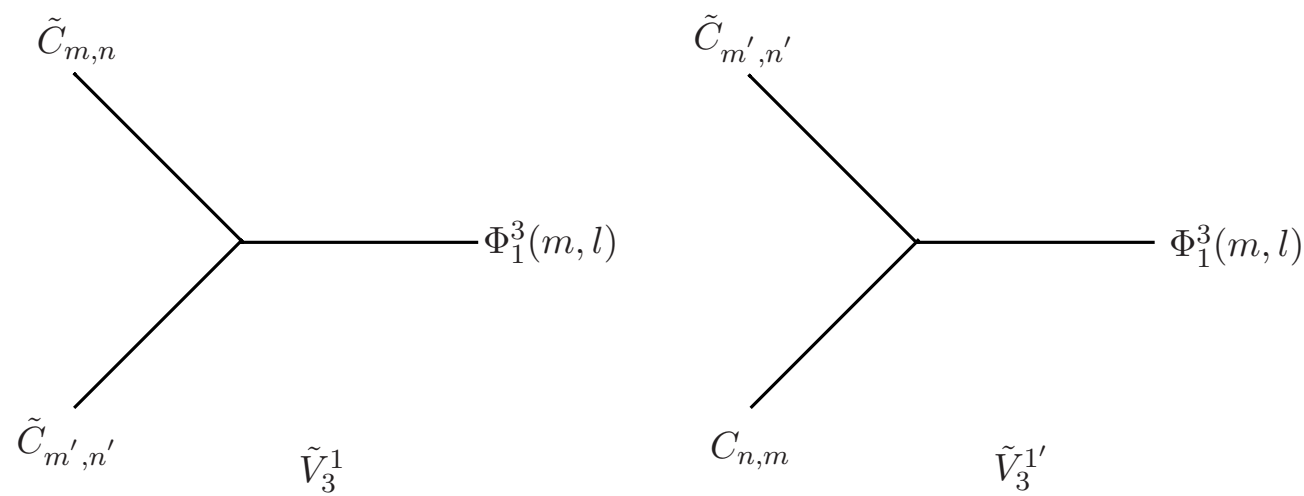

Figure 47. $\tilde{V}_{3}^{1}$ and $\tilde{V}_{3}^{1^{\prime}}$ vertices.
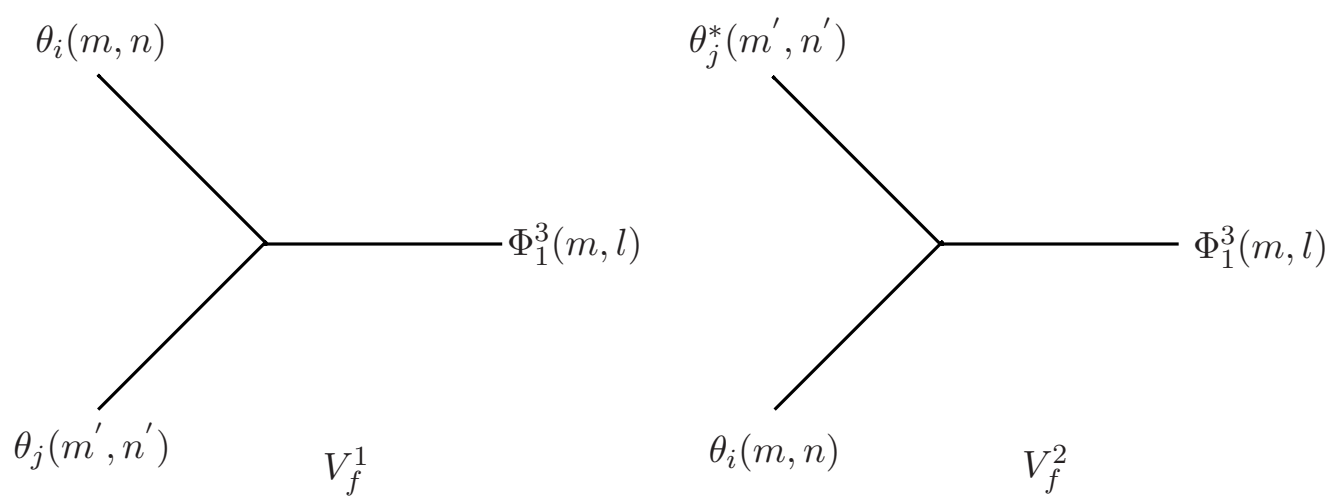

Figure 48. The fermionic vertices $V_{f}^{1}$ and $V_{f}^{2}$ for $\Phi_{1}^{3}-\Phi_{1}^{3}$ amplitudes.

\section{E.2 $\Phi_{I}^{3}, I \neq 1$ vertices}

We list here the vertices for the one-loop finite temperature mass-squared corrections for the massless field $\Phi_{I}^{3}(m, l), I \neq 1$.

$$
\begin{aligned}
V_{1}^{I} & =-\frac{N}{2 g^{2}} G_{1}^{I}\left(l, l^{\prime}, n, n^{\prime}\right) \delta_{w+w^{\prime}+m+m^{\prime}} \quad \text { (figure 49) } \\
G_{1}^{I}\left(l, l^{\prime}, n, n^{\prime}\right) & =\sqrt{q} \int d x e^{-q x^{2}} H_{n}(x) H_{n^{\prime}}(x) e^{i l x} e^{i l^{\prime} x}
\end{aligned}
$$




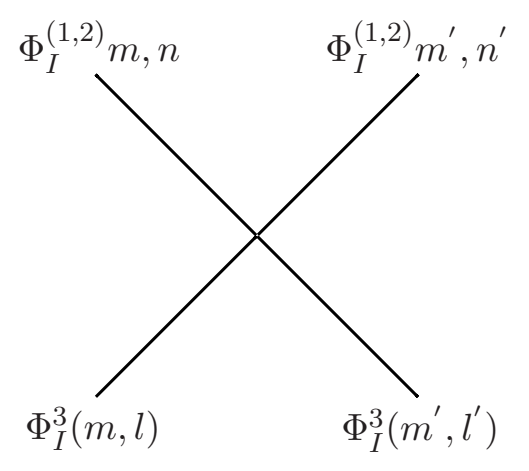

$V_{1}^{I}$

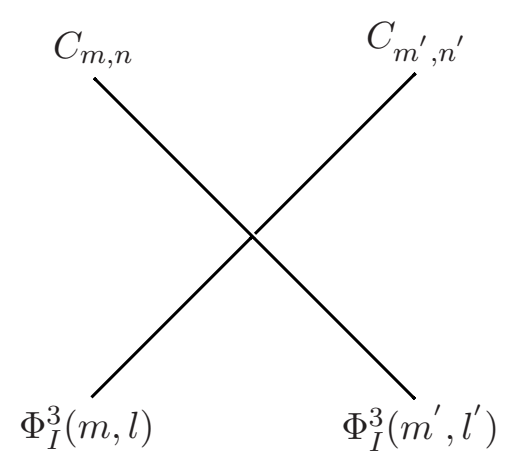

$V_{2}^{I}$

Figure 49. $V_{1}^{I}$ and $V_{2}^{I}$ vertices.

$$
\begin{aligned}
& V_{2}^{I}=-\frac{N}{2 g^{2}} G_{2}^{I}\left(l, l^{\prime}, n, n^{\prime}\right) \delta_{w+w^{\prime}+m+m^{\prime}} \quad \text { (figure 49) } \\
& G_{2}^{I}\left(l, l^{\prime}, n, n^{\prime}\right)=\sqrt{q} \int d x\left[A_{n}(x) A_{n^{\prime}}(x)+\phi_{n}(x) \phi_{n^{\prime}}(x)\right] e^{i l x} e^{i l^{\prime} x} \\
& \tilde{V}_{2}^{I}=-\frac{N}{2 g^{2}} \tilde{G}_{2}^{I}\left(l, l^{\prime}, n, n^{\prime}\right) \delta_{w+w^{\prime}+m+m^{\prime}} \quad \text { (figure 50) } \\
& \tilde{G}_{2}^{I}\left(l, l^{\prime}, n, n^{\prime}\right)=\sqrt{q} \int d x\left[\tilde{A}_{n}(x) \tilde{A}_{n^{\prime}}(x)+\tilde{\phi}_{n}(x) \tilde{\phi}_{n^{\prime}}(x)\right] e^{i l x} e^{i l^{\prime} x} \\
& V_{3}^{I}=-\frac{N^{3 / 2}}{g^{2}} G_{3}^{I}\left(l, n, n^{\prime}\right) \beta \delta_{w+m+m^{\prime}} \quad \text { (figure 51) } \\
& G_{3}^{I}\left(l, n, n^{\prime}\right)=\int d x e^{i l x}\left[\partial_{x}\left(e^{-q x^{2} / 2} H_{n^{\prime}}(x)\right) A_{n}(x)-i l e^{-q x^{2} / 2} A_{n}(x) H_{n^{\prime}}(x)\right. \\
& \left.-q x \phi_{n}(x) e^{-q x^{2} / 2} H_{n^{\prime}}(x)\right] \\
& \tilde{V}_{3}^{I}=-\frac{N^{3 / 2}}{g^{2}} \tilde{G}_{3}^{I}\left(l, n, n^{\prime}\right) \beta \delta_{w+m+m^{\prime}} \quad \text { (figure 51) } \\
& \tilde{G}_{3}^{I}\left(l, n, n^{\prime}\right)=\int d x e^{i l x}\left[\partial_{x}\left(e^{-q x^{2} / 2} H_{n^{\prime}}(x)\right) \tilde{A}_{n}(x)-i l e^{-q x^{2} / 2} \tilde{A}_{n}(x) H_{n^{\prime}}(x)\right. \\
& \left.-q x \tilde{\phi}_{n}(x) e^{-q x^{2} / 2} H_{n^{\prime}}(x)\right] \\
& V_{f}^{I}=i \frac{N}{g^{2}} G_{f}^{I}\left(l, n, n^{\prime}\right) \delta_{w+m+m^{\prime}} \quad \text { (figure 52) } \\
& G_{f}^{I}\left(l, n, n^{\prime}\right)=\sqrt{q} \int d x e^{i l x}\left[R_{n}(x) L_{n^{\prime}}+L_{n}(x) R_{n^{\prime}}(x)\right]
\end{aligned}
$$

\section{E.3 $\quad A_{x}^{3}$ vertices}

In this section we write down the various vertices needed for computing the two point $A_{x}^{3}$ amplitude.

$$
V_{1}^{A}=-\frac{N}{2 g^{2}} G_{1}^{A}\left(n, n^{\prime}, l, l^{\prime}\right) \delta_{w+w^{\prime}+m+m^{\prime}} \quad \text { (figure 53) }
$$




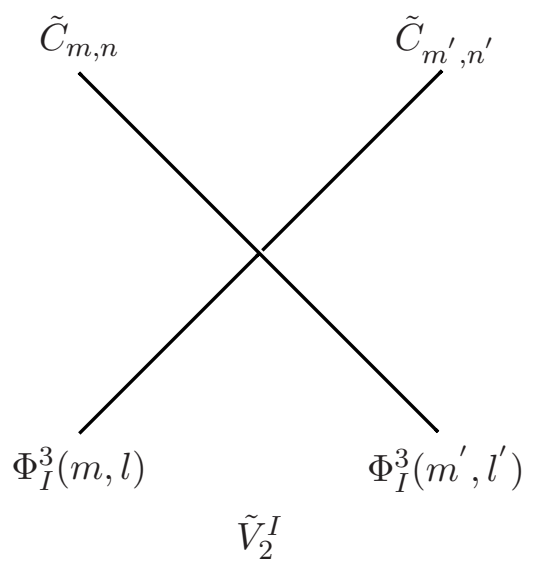

Figure 50. $\tilde{V}_{2}^{I}$ vertex.

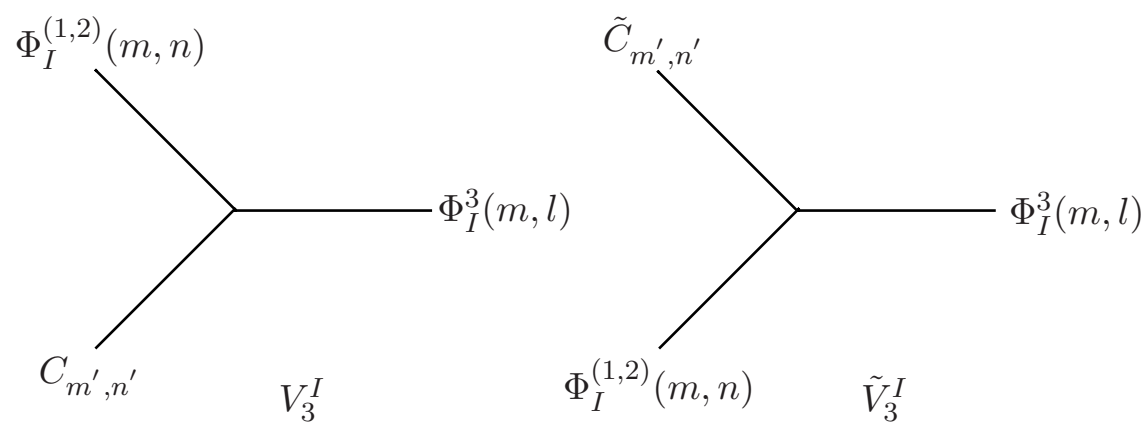

Figure 51. $V_{3}^{I}$ and $\tilde{V}_{3}^{I}$ vertices.

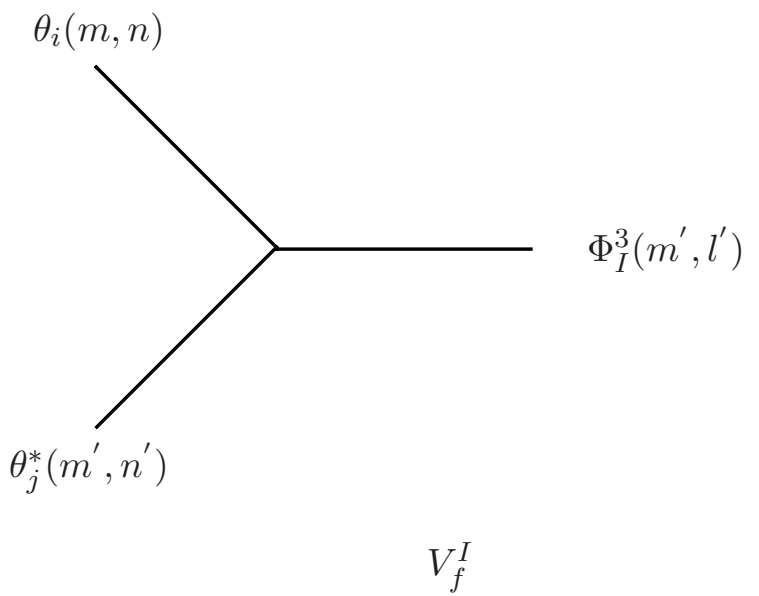

Figure 52. $V_{f}^{I}$ vertex. 


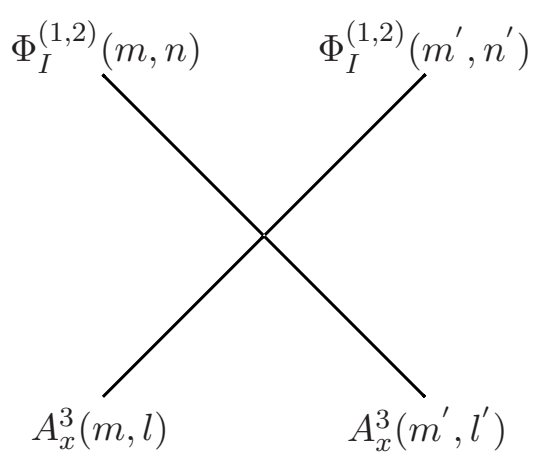

$V_{1}^{A}$

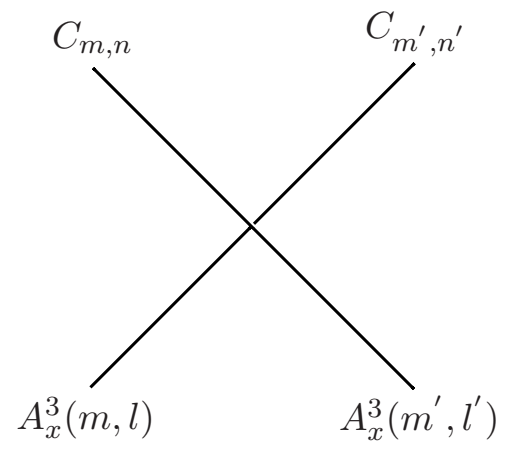

$V_{2}^{A}$

Figure 53. $V_{1}^{A}$ and $V_{2}^{A}$ vertices.

$$
\begin{aligned}
G_{1}^{A}\left(n, n^{\prime} l, l^{\prime}\right)= & \sqrt{q} \int d x e^{-q x^{2}} H_{n}(\sqrt{q} x) H_{n^{\prime}}(\sqrt{q} x) e^{i\left(l+l^{\prime}\right) x} \\
V_{2}^{A}= & -\frac{N}{2 g^{2}} G_{2}^{A}\left(n, n^{\prime}, l, l^{\prime}\right) \delta_{w+w^{\prime}+m+m^{\prime}} \quad \text { (figure 53) } \\
G_{2}^{A}\left(n, n^{\prime}, l, l^{\prime}\right)= & \sqrt{q} \int d x \phi_{n}(x) \phi_{n^{\prime}}(x) e^{i\left(l+l^{\prime}\right) x} \\
\tilde{V}_{2}^{A}= & -\frac{N}{2 g^{2}} \tilde{G}_{2}^{A}\left(n, n^{\prime}, l, l^{\prime}\right) \delta_{w+w^{\prime}+m+m^{\prime}} \quad \text { (figure 54) } \\
\tilde{G}_{2}^{A}\left(n, n^{\prime}, l, l^{\prime}\right)= & \sqrt{q} \int d x \tilde{\phi}_{n}(x) \tilde{\phi}_{n^{\prime}}(x) e^{i\left(l+l^{\prime}\right) x} \\
V_{3}^{A}= & -\frac{N^{3 / 2}}{g^{2}} G_{3}^{A}\left(n, n^{\prime}, l\right) \beta \delta_{w+m+m^{\prime}} \quad(\text { figure 55) } \\
G_{3}^{A}\left(n, n^{\prime}, l\right)= & \int d x\left[\partial_{x}\left(\phi_{n^{\prime}}(x)\right) \phi_{n}(x)-\partial_{x}\left(\phi_{n}(x)\right) \phi_{n^{\prime}}(x)\right. \\
& \left.+q x A_{n^{\prime}}(x) \phi_{n}(x)-q x A_{n}(x) \phi_{n^{\prime}}(x)\right] e^{i l x} \\
\tilde{V}_{3}^{A}= & -\frac{N^{3 / 2}}{g^{2}} \tilde{G}_{3}^{A}\left(n, n^{\prime}, l\right) \beta \delta_{w+m+m^{\prime}} \quad(\text { figure 55) } \\
\tilde{G}_{3}^{A}\left(n, n^{\prime}, l\right)= & \int d x\left[\partial_{x}\left(\tilde{\phi}_{n^{\prime}}(x)\right) \tilde{\phi}_{n}(x)-\partial_{x}\left(\tilde{\phi}_{n}(x)\right) \tilde{\phi}_{n^{\prime}}(x)\right. \\
& \left.+q x \tilde{A}_{n^{\prime}}(x) \tilde{\phi}_{n}(x)-q x \tilde{A}_{n}(x) \tilde{\phi}_{n^{\prime}}(x)\right] e^{i l x} \\
V_{4}^{A}= & -\frac{N^{3 / 2}}{g^{2}} G_{4}^{A}\left(n, n^{\prime}, l\right) \beta \delta_{w+m+m^{\prime}} \quad(\text { figure 56) } \\
\tilde{G}_{3}^{A^{\prime}}\left(n, n^{\prime}, l\right)= & \int d x\left[\partial_{x}\left(\tilde{\phi}_{n^{\prime}}(x)\right) \phi_{n}(x)-\partial_{x}\left(\phi_{n}(x)\right) \tilde{\phi}_{n^{\prime}}(x)\right. \\
\tilde{V}_{3}^{A^{\prime}}= & -\frac{N^{3 / 2}}{g^{2}} \tilde{G}_{3}^{A^{\prime}}\left(n, n^{\prime}, l\right) \beta \delta_{w+m+m^{\prime}} \quad(\text { figure 56) } \\
& \left.+q x \tilde{A}_{n^{\prime}}(x) \phi_{n}(x)-\tilde{\phi}_{n^{\prime}}(x) A_{n}(x)\right] e^{i l x} \\
&
\end{aligned}
$$




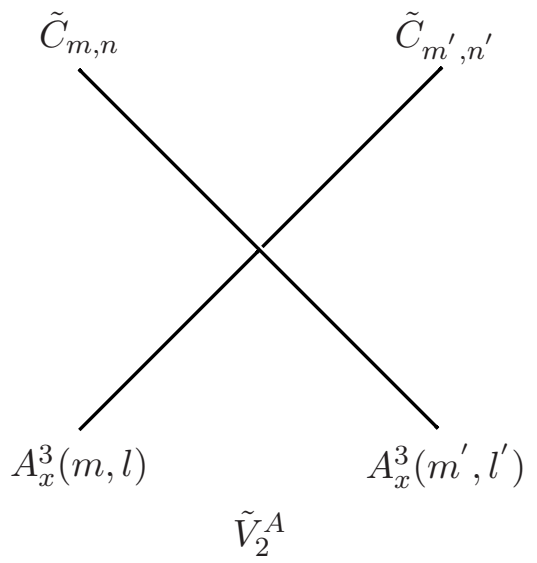

Figure 54. $\tilde{V}_{2}^{A}$ vertex.
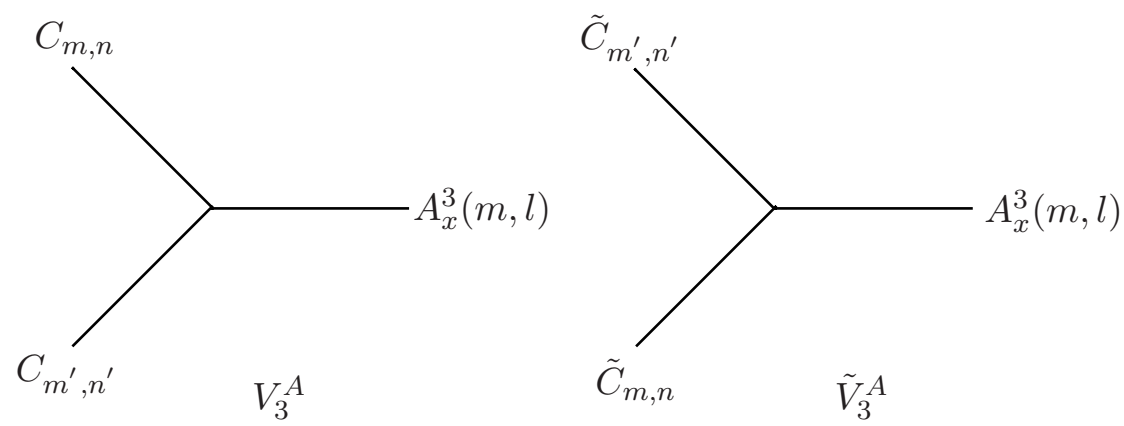

Figure 55. $V_{3}^{A}$ and $\tilde{V}_{3}^{A}$ vertices.

$$
\begin{aligned}
G_{4}^{A}\left(n, n^{\prime}, l\right)= & \int d x e^{-q x^{2} / 2}\left[\partial_{x}\left(e^{-q x^{2} / 2} H_{n^{\prime}}(x)\right) H_{n}(x)\right. \\
& \left.-\partial_{x}\left(e^{-q x^{2} / 2} H_{n}(x)\right) H_{n^{\prime}}(x)\right] e^{i l x} \\
V_{f}^{A 1}= & i \frac{N}{g^{2}} G_{4}^{A 1}\left(n, n^{\prime}, l\right) \beta \delta_{w+m+m^{\prime}} \quad \text { (figure 57) } \\
G_{f}^{A 1}\left(n, n^{\prime}, l\right)= & \sqrt{q} \int d x\left[L_{n}(x) L_{n^{\prime}}(x)-R_{n}(x) R_{n^{\prime}}(x)\right] e^{i l x} \\
V_{f}^{A 2}= & i \frac{N}{g^{2}} G_{4}^{A 2}\left(n, n^{\prime}, l\right) \beta \delta_{w+m+m^{\prime}} \quad \text { (figure 57) } \\
G_{f}^{A 2}\left(n, n^{\prime}, l\right)= & \sqrt{q} \int d x\left[L_{n}(x) L_{n^{\prime}}^{*}(x)-R_{n}(x) R_{n^{\prime}}^{*}(x)\right] e^{i l x}
\end{aligned}
$$

\section{F Matsubara sums}

In this appendix we show some sample computations showing sums over Matsubara frequencies. Let us evaluate the sum over $m$ in the propagator

$$
\frac{1}{\beta} \sum_{n=2, m=-\infty}^{\infty} \frac{1}{\omega_{m}^{2}+\lambda_{n}}
$$

where $\omega_{m}=2 m \pi / \beta$. 


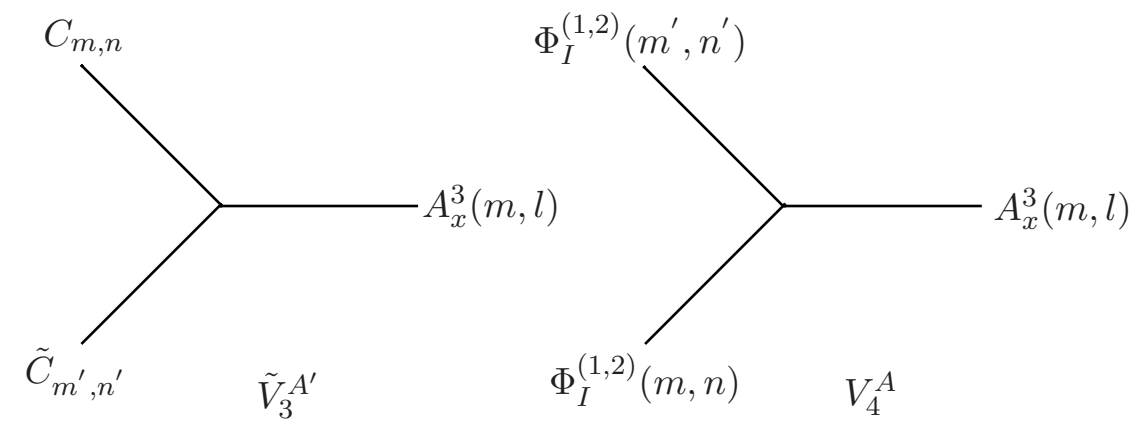

Figure 56. $\tilde{V}_{3}^{A^{\prime}}$ and $V_{4}^{A}$ vertices.
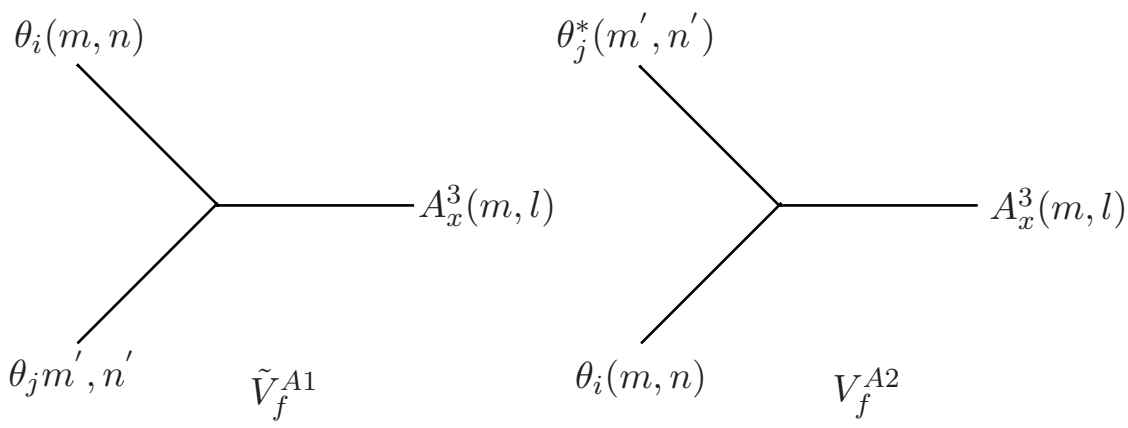

Figure 57. $V_{f}^{A 1}$ and $V_{f}^{A 2}$ vertices.

Following [42] we convert the sum over $m$ into a contour integral as follows. By writing $p_{0}=i \omega_{m}$, define the function

$$
f\left(p_{0}\right)=-\frac{1}{p_{0}^{2}-\lambda_{n}} .
$$

The function $f\left(p_{0}\right)$ does not have poles on the imaginary axis. We multiply it by a function with simple poles on the imaginary axis at values $p_{0}=\frac{2 i m \pi}{\beta}$ and analytic and bounded otherwise. A function with this property is $\operatorname{coth}\left(p_{0} \beta / 2\right)$. The sum over $m$ in (F.1) can now be reproduced from the contour integral

$$
\frac{1}{2 \pi i \beta} \oint d p_{0}\left(\frac{\beta}{2}\right) \operatorname{coth}\left(\frac{p_{0} \beta}{2}\right) f\left(p_{0}\right)
$$

where the contour is the sum over the contours $C_{n}$ shown in figure 58(a). The $C_{n}$ 's can now be deformed into the contour $\Gamma$. The contour integral can then be written in terms of line integrals as

$$
\frac{1}{2 \pi i} \int_{i \infty-\epsilon}^{-i \infty-\epsilon} d p_{0} f\left(p_{0}\right)\left(-\frac{1}{2}-\frac{1}{e^{-p_{0} \beta}-1}\right)+\frac{1}{2 \pi i} \int_{-i \infty+\epsilon}^{i \infty+\epsilon} d p_{0} f\left(p_{0}\right)\left(\frac{1}{2}+\frac{1}{e^{p_{0} \beta}-1}\right)
$$

since the function $f\left(p_{0}\right)$ vanishes for $p_{0}= \pm i \infty$.

In (F.4) the frequency sum separates into the zero-temperature part and the temperature-dependent part for both the integrals. The line integrals above can now be evaluated using the contour integrals over the contours $\Gamma_{1}$ and $\Gamma_{2}$ in figure 58(b). This is because the line integral over rest of the rectangle vanishes when the length of the sides 


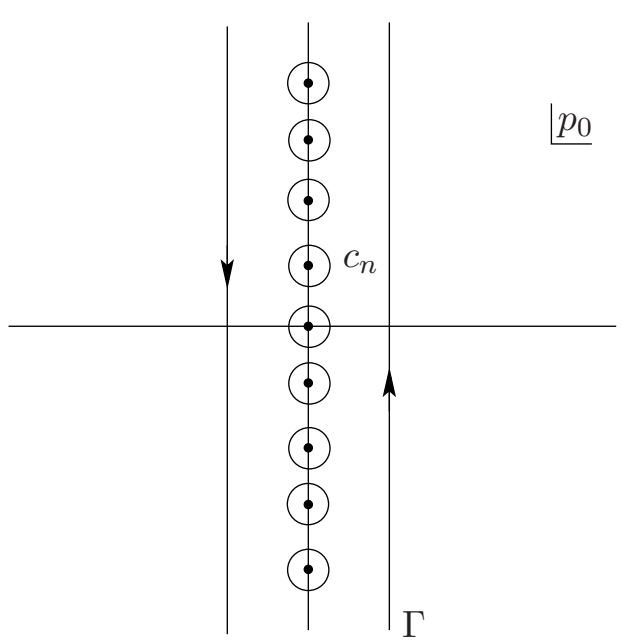

(a)

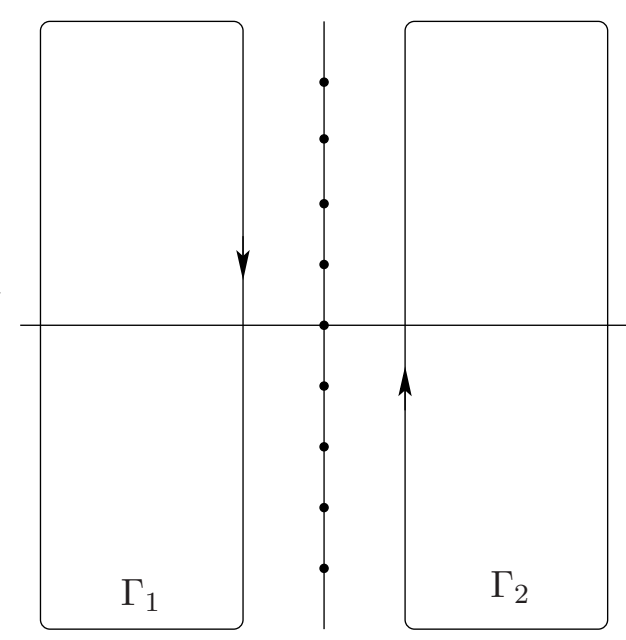

(b)

Figure 58. Contours for doing the Matsubara sums.

are taken to infinity. These contour integrals now have contributions only from the poles of $f\left(p_{0}\right)$ at $p_{0}= \pm \sqrt{\lambda_{n}}$. Thus,

$$
\frac{1}{\beta} \sum_{n=2, m=-\infty}^{\infty} \frac{1}{\omega_{m}^{2}+\lambda_{n}}=\sum_{n=2} \frac{1}{\sqrt{\lambda_{n}}}\left(\frac{1}{2}+\frac{1}{e^{\sqrt{\lambda_{n}}} \beta-1}\right) .
$$

Similarly using the above formula, the several bosonic propagators upon being summed over the Matsubara frequency $\omega_{m}$ are,

$$
\begin{gathered}
\frac{1}{\beta} \sum_{n=2, m=-\infty}^{\infty} \frac{1}{\omega_{m}^{2}+\gamma_{n}}=\sum_{n=2}^{\infty} \frac{1}{\sqrt{\gamma_{n}}}\left(\frac{1}{2}+\frac{1}{e^{\sqrt{\gamma_{n}} \beta}-1}\right) \\
\frac{1}{\beta} \sum_{n=2, n^{\prime}=2, m=-\infty}^{\infty} \int_{-\infty}^{\infty} \frac{d l}{2 \pi \sqrt{q}} \frac{1}{\omega_{m}^{2}+l^{2}}=\int_{-\infty}^{\infty} \frac{d l}{2 \pi \sqrt{q}} \frac{1}{l}\left(\frac{1}{2}+\frac{1}{e^{l \beta}-1}\right) \\
\sum_{m=2, n^{\prime}=2}^{\infty} \frac{1}{\left.\bar{\omega}_{n}^{2}+\gamma_{n}\right)\left(\omega_{m}^{2}+\lambda_{n^{\prime}}\right)}= \\
\left.\sum_{n=2, m=-\infty}^{\infty} \int_{-\infty}^{\infty} \frac{1}{2 \pi \sqrt{q}}\left(\frac{1}{\lambda_{n^{\prime}}}+\frac{1}{e^{\sqrt{\lambda_{n^{\prime}}} \beta}-1}\right)-\frac{1}{\sqrt{\gamma_{n}}}\left(\frac{1}{2}+\frac{1}{e^{\sqrt{\gamma_{n}} \beta}-1}\right)\right) \\
\sum_{n=2}^{\infty} \int_{-\infty}^{\infty} \frac{d l}{2 \pi \sqrt{q}} \frac{1}{l^{2}-\lambda}\left(\frac{1}{\lambda_{n}}\left(\frac{1}{2}+\frac{1}{e^{\sqrt{\lambda_{n}} \beta}-1}\right)-\frac{1}{l}\left(\frac{1}{2}+\frac{1}{e^{l \beta}-1}\right)\right) \\
\frac{1}{\beta} \sum_{n=2, m=-\infty}^{\infty} \int_{-\infty}^{\infty} \frac{d l}{2 \pi \sqrt{q}} \frac{1}{\left(\omega_{m}^{2}+l^{2}\right)\left(\omega_{m}^{2}+\gamma_{n}\right)}= \\
\sum_{n=2}^{\infty} \int_{-\infty}^{\infty} \frac{d l}{2 \pi \sqrt{q}} \frac{1}{l^{2}-\gamma_{n}}\left(\frac{1}{\gamma_{n}}\left(\frac{1}{2}+\frac{1}{e^{\sqrt{\gamma_{n}} \beta}-1}\right)-\frac{1}{l}\left(\frac{1}{2}+\frac{1}{e^{l \beta}-1}\right)\right) .
\end{gathered}
$$


The last three propagators in are mixed propagators. So we have decomposed them into partial fractions and then and have done the sum separately.

The fermions due to their anti-periodic boundary conditions along the Euclidean time direction have have their propagators with $\omega_{m}=\frac{(2 m+1) \pi}{\beta}$. The sum over the odd integers can be performed by converting the sum into a contour integral as above. The only change here is that we must introduce $\tanh \left(p_{0} \beta / 2\right)$. Thus,

$$
\begin{aligned}
\frac{1}{\beta} \sum_{m=-\infty}^{\infty} \frac{1}{\omega_{m}^{2}+\lambda_{n}^{\prime}} & =\frac{1}{2 \pi i \beta} \oint d p_{0}\left(\frac{\beta}{2}\right) \tanh \left(\frac{p_{0} \beta}{2}\right) f\left(p_{0}\right) \\
& =\frac{1}{\sqrt{\lambda_{n}^{\prime}}}\left(\frac{1}{2}-\frac{1}{e^{\sqrt{\lambda_{n}^{\prime}} \beta}+1}\right) .
\end{aligned}
$$

Similarly using this result we can do the sum over the Matsubara frequencies for the following,

$$
\begin{aligned}
& \sum_{n=0, m=-\infty}^{\infty} \int_{-\infty}^{\infty} \frac{d l}{2 \pi \sqrt{q}} \frac{1}{\left(i \omega_{m}+\sqrt{\lambda_{n}^{\prime}}\right)\left(i \omega_{m} \pm l\right)}= \\
& \quad-\sum_{n=0, m=-\infty}^{\infty} \int_{-\infty}^{\infty} \frac{d l}{4 \pi \sqrt{q}}\left(\frac{1}{\left(\omega_{m}^{2}+\lambda_{n}^{\prime}\right)}+\frac{1}{\left(\omega_{m}^{2}+l^{2}\right)}-\frac{l^{2}+\lambda_{n}}{\left(\omega_{m}^{2}+\lambda_{n}^{\prime}\right)\left(\omega_{m}^{2}+l^{2}\right)}\right) .
\end{aligned}
$$

We can now do the sum over each of the terms separately, which gives

$$
\sum_{n} \int \frac{d l}{2 \pi \sqrt{q}}\left(\frac{-\beta \tanh \left(\frac{\beta l}{2}\right)+\beta \tanh \left(\frac{1}{2} \beta \sqrt{\lambda_{n}^{\prime}}\right)}{2\left(l-\sqrt{\lambda_{n}^{\prime}}\right)}\right) .
$$

Open Access. This article is distributed under the terms of the Creative Commons Attribution License (CC-BY 4.0), which permits any use, distribution and reproduction in any medium, provided the original author(s) and source are credited.

\section{References}

[1] S. Kalyana Rama, S. Sarkar, B. Sathiapalan and N. Sircar, Strong Coupling BCS Superconductivity and Holography, Nucl. Phys. B 852 (2011) 634 [arXiv:1104.2843] [INSPIRE].

[2] H. Liu, J. McGreevy and D. Vegh, Non-Fermi liquids from holography, Phys. Rev. D 83 (2011) 065029 [arXiv:0903.2477] [InSPIRE].

[3] T. Faulkner, H. Liu, J. McGreevy and D. Vegh, Emergent quantum criticality, Fermi surfaces and $A d S_{2}$, Phys. Rev. D 83 (2011) 125002 [arXiv:0907.2694] [inSPIRE].

[4] T. Faulkner and J. Polchinski, Semi-Holographic Fermi Liquids, JHEP 06 (2011) 012 [arXiv: 1001.5049] [INSPIRE].

[5] D. Nickel and D.T. Son, Deconstructing holographic liquids, New J. Phys. 13 (2011) 075010 [arXiv: 1009.3094] [INSPIRE].

[6] T. Faulkner, H. Liu and M. Rangamani, Integrating out geometry: Holographic Wilsonian $R G$ and the membrane paradigm, JHEP 08 (2011) 051 [arXiv:1010.4036] [INSPIRE]. 
[7] I. Heemskerk and J. Polchinski, Holographic and Wilsonian Renormalization Groups, JHEP 06 (2011) 031 [arXiv: 1010.1264] [INSPIRE].

[8] C.P. Herzog, P. Kovtun, S. Sachdev and D.T. Son, Quantum critical transport, duality and M-theory, Phys. Rev. D 75 (2007) 085020 [hep-th/0701036] [INSPIRE].

[9] F. Denef, S.A. Hartnoll and S. Sachdev, Quantum oscillations and black hole ringing, Phys. Rev. D 80 (2009) 126016 [arXiv:0908.1788] [INSPIRE].

[10] S.S. Gubser, TASI lectures: Collisions in anti-de Sitter space, conformal symmetry and holographic superconductors, arXiv:1012.5312 [INSPIRE].

[11] S.S. Gubser, S.S. Pufu and F.D. Rocha, Quantum critical superconductors in string theory and M-theory, Phys. Lett. B 683 (2010) 201 [arXiv:0908.0011] [INSPIRE].

[12] S.A. Hartnoll, C.P. Herzog and G.T. Horowitz, Building a Holographic Superconductor, Phys. Rev. Lett. 101 (2008) 031601 [arXiv:0803.3295] [INSPIRE].

[13] S.A. Hartnoll, C.P. Herzog and G.T. Horowitz, Holographic Superconductors, JHEP 12 (2008) 015 [arXiv:0810.1563] [INSPIRE].

[14] Y. Nambu and G. Jona-Lasinio, Dynamical Model of Elementary Particles Based on an Analogy with Superconductivity. I, Phys. Rev. 122 (1961) 345 [INSPIRE].

[15] Y. Nambu and G. Jona-Lasinio, Dynamical model of elementary particles based on an analogy with superconductivity. II, Phys. Rev. 124 (1961) 246 [INSPIRE].

[16] E. Witten, Anti-de Sitter space, thermal phase transition and confinement in gauge theories, Adv. Theor. Math. Phys. 2 (1998) 505 [hep-th/9803131] [INSPIRE].

[17] A. Karch and E. Katz, Adding flavor to AdS/CFT, JHEP 06 (2002) 043 [hep-th/0205236] [INSPIRE].

[18] M. Kruczenski, D. Mateos, R.C. Myers and D.J. Winters, Towards a holographic dual of large- $N_{c} Q C D, J H E P 05$ (2004) 041 [hep-th/0311270] [INSPIRE].

[19] T. Sakai and J. Sonnenschein, Probing flavored mesons of confining gauge theories by supergravity, JHEP 09 (2003) 047 [hep-th/0305049] [INSPIRE].

[20] J. Erlich, E. Katz, D.T. Son and M.A. Stephanov, QCD and a holographic model of hadrons, Phys. Rev. Lett. 95 (2005) 261602 [hep-ph/0501128] [INSPIRE].

[21] A. Karch, E. Katz, D.T. Son and M.A. Stephanov, Linear confinement and AdS/QCD, Phys. Rev. D 74 (2006) 015005 [hep-ph/0602229] [INSPIRE].

[22] E. Antonyan, J.A. Harvey, S. Jensen and D. Kutasov, NJLS and QCD from string theory, hep-th/0604017 [INSPIRE].

[23] E. Antonyan, J.A. Harvey and D. Kutasov, The Gross-Neveu Model from String Theory, Nucl. Phys. B 776 (2007) 93 [hep-th/0608149] [INSPIRE].

[24] E. Antonyan, J.A. Harvey and D. Kutasov, Chiral symmetry breaking from intersecting D-branes, Nucl. Phys. B 784 (2007) 1 [hep-th/0608177] [INSPIRE].

[25] T. Sakai and S. Sugimoto, Low energy hadron physics in holographic QCD, Prog. Theor. Phys. 113 (2005) 843 [hep-th/0412141] [INSPIRE].

[26] H. Hata, T. Sakai, S. Sugimoto and S. Yamato, Baryons from instantons in holographic QCD, Prog. Theor. Phys. 117 (2007) 1157 [hep-th/0701280] [INSPIRE]. 
[27] O. Bergman, G. Lifschytz and M. Lippert, Holographic Nuclear Physics, JHEP 11 (2007) 056 [arXiv:0708.0326] [INSPIRE].

[28] S. Kobayashi, D. Mateos, S. Matsuura, R.C. Myers and R.M. Thomson, Holographic phase transitions at finite baryon density, JHEP 02 (2007) 016 [hep-th/0611099] [INSPIRE].

[29] O. Aharony, J. Sonnenschein and S. Yankielowicz, A holographic model of deconfinement and chiral symmetry restoration, Annals Phys. 322 (2007) 1420 [hep-th/0604161] [INSPIRE].

[30] O. Bergman, S. Seki and J. Sonnenschein, Quark mass and condensate in HQCD, JHEP 12 (2007) 037 [arXiv:0708.2839] [INSPIRE].

[31] A. Dhar and P. Nag, Sakai-Sugimoto model, Tachyon Condensation and Chiral symmetry Breaking, JHEP 01 (2008) 055 [arXiv:0708.3233] [INSPIRE].

[32] A. Dhar and P. Nag, Tachyon condensation and quark mass in modified Sakai-Sugimoto model, Phys. Rev. D 78 (2008) 066021 [arXiv:0804.4807] [InSPIRE].

[33] K. Hashimoto and S. Nagaoka, Recombination of intersecting D-branes by local tachyon condensation, JHEP 06 (2003) 034 [hep-th/0303204] [INSPIRE].

[34] K. Hashimoto and W. Taylor, Strings between branes, JHEP 10 (2003) 040 [hep-th/0307297] [INSPIRE].

[35] F.T.J. Epple and D. Lüst, Tachyon condensation for intersecting branes at small and large angles, Fortsch. Phys. 52 (2004) 367 [hep-th/0311182] [INSPIRE].

[36] M. Berkooz, M.R. Douglas and R.G. Leigh, Branes intersecting at angles, Nucl. Phys. B 480 (1996) 265 [hep-th/9606139] [inSPIRE].

[37] A. Hashimoto and W. Taylor, Fluctuation spectra of tilted and intersecting D-branes from the Born-Infeld action, Nucl. Phys. B 503 (1997) 193 [hep-th/9703217] [INSPIRE].

[38] S. Nagaoka, Fluctuation analysis of nonAbelian Born-Infeld action in the background intersecting D-branes, Prog. Theor. Phys. 110 (2004) 1219 [hep-th/0307232] [INSPIRE].

[39] N. Jokela, M. Jarvinen and S. Nowling, Winding effects on brane/anti-brane pairs, JHEP 07 (2009) 085 [arXiv:0901.0281] [INSPIRE].

[40] N. Jokela and M. Lippert, Inhomogeneous tachyon dynamics and the zipper, JHEP 08 (2009) 024 [arXiv:0906.0317] [INSPIRE].

[41] M.B. Green, J.H. Schwarz and E. Witten, Superstring Theory. Volume 1: Introduction, Cambridge Monographs on Mathematical Physics, Cambridge University Press.

[42] J. Kapusta, Finite Temperature Field Theory, Cambridge Monographs on Mathematical Physics, Cambridge University Press. 